\title{
Emergence of irregular activity in networks of strongly coupled conductance-based neurons
}

\author{
A. Sanzeni, ${ }^{1,2}$ M.H. Histed, ${ }^{2}$ and N. Brunel ${ }^{1,3}$ \\ ${ }^{1}$ Department of Neurobiology, Duke University, Durham, NC, USA \\ ${ }^{2}$ National institute of Mental Health Intramural Program, NIH, Bethesda, MD, USA \\ ${ }^{3}$ Department of Physics, Duke University, Durham, NC, USA
}

\begin{abstract}
Cortical neurons are characterized by irregular firing and a broad distribution of rates. The balanced state model explains these observations with a cancellation of mean excitatory and inhibitory currents, which makes fluctuations drive firing. In networks of neurons with current-based synapses, the balanced state emerges dynamically if coupling is strong, i.e. if the mean number of synapses per neuron $K$ is large and synaptic efficacy is of order $1 / \sqrt{K}$. When synapses are conductancebased, current fluctuations are suppressed when coupling is strong, questioning the applicability of the balanced state idea to biological neural networks. We analyze networks of strongly coupled conductance-based neurons and show that asynchronous irregular activity and broad distributions of rates emerge if synaptic efficacy is of order $1 / \log (K)$. In such networks, unlike in the standard balanced state model, current fluctuations are small and firing is maintained by a drift-diffusion balance. This balance emerges dynamically, without fine tuning, if inputs are smaller than a critical value, which depends on synaptic time constants and coupling strength, and is significantly more robust to connection heterogeneities than the classical balanced state model. Our analysis makes experimentally testable predictions of how the network response properties should evolve as input increases.
\end{abstract}

\section{INTRODUCTION}

Each neuron in cortex receives inputs from hundreds to thousands of pre-synaptic neurons. If these inputs were to sum to produce a large net current, the central limit theorem argues that fluctuations should be small compared to the mean, leading to regular firing, as observed during in vitro experiments under constant current injection 1, 2. Cortical activity, however, is highly irregular, with a coefficient of variation of interspike intervals (CV of ISI) close to one 3, 4. To explain the observed irregularity, it has been proposed that neural networks operate in a balanced state, where strong feedforward and recurrent excitatory inputs are canceled by recurrent inhibition and firing is driven by fluctuations 5, 6. At the single neuron level, in order for this state to emerge, input currents must satisfy two constraints. First, excitatory and inhibitory currents must be fine tuned so to produce an average input below threshold. Specifically, if $K$ and $J$ represent the average number of input connections per neuron and synaptic efficacy, respectively, the difference between excitatory and inhibitory presynaptic inputs must be of order $1 / K J$. Second, input fluctuations should be large enough to drive firing.

It has been shown that the balanced state emerges dynamically (without fine tuning) in randomly connected networks of binary units [7, 8, and networks of currentbased spiking neurons [9, 10, provided that coupling is strong, and recurrent inhibition is powerful enough to counterbalance instabilities due to recurrent excitation. However, these results have all been derived assuming that the firing of a presynaptic neuron produces a fixed amount of synaptic current, hence neglecting the dependence of synaptic current on the membrane potential, a key aspect of neuronal biophysics. In real synapses, synaptic inputs are mediated by changes in conductance, due to opening of synaptic receptor-channels on the membrane, and synaptic currents are proportional to the product of synaptic conductance and a driving force which depends on the membrane potential. Models that incorporate this description are referred to as 'conductance-based synapses'.

Large synaptic conductances has been shown to have major effects on the stationary 11 and dynamical 12 response of single cells, and form the basis of the 'highconductance state' $[13$ 19] that has been argued to describe well in vivo data 20 22] (but see 23] and Discussion). At the network level, conductance modulation plays a role in controlling signal propagation 24, input summation 25], and firing statistics 26. However, most of the previously mentioned studies rely exclusively on numerical simulations, and in spite of a few attempts at analytical descriptions of networks of conductance-based neurons [17, 27 31, an understanding of the behavior of such networks when coupling is strong is still lacking.

Here, we investigate networks of strongly coupled conductance-based neurons. We find that, for synapses of order $1 / \sqrt{K}$, fluctuations are too weak to sustain firing, questioning the relevance of the balanced state idea to cortical dynamics. Our analysis, on the other hand, shows that stronger synapses (of order $1 / \log (K)$ ) generate irregular firing when coupling is strong. We characterize the properties of networks with such a scaling, showing that they match properties observed in cortex, and discuss constraints induced by synaptic time constant. The model generates qualitatively different predictions compared to the current-based model, which could be tested experimentally. 


\section{MODELS OF SINGLE NEURON AND NETWORK DYNAMICS}

Membrane potential dynamics. We study the ${ }_{122}$ dynamics of networks of leaky integrate-and-fire (LIF) ${ }^{123}$ neurons with conductance-based synaptic inputs. The ${ }^{124}$ membrane potential $V_{j}$ of the $j$-th neuron in the net- ${ }^{125}$ work follows the equation

$$
\mathcal{C}_{j} \frac{d V_{j}}{d t}=-\sum_{A=L, E, I} g_{A}^{j}\left(V_{j}-E_{A}\right),
$$

where $\mathcal{C}_{j}$ is the neuronal capacitance; $E_{L}, E_{E}$ and $E_{I}$ are the reversal potentials of the leak, excitatory and inhibitory currents; while $g_{L}^{j}, g_{E}^{j}$ and $g_{I}^{j}$ are the leak, excitatory and inhibitory conductances. Assuming instantaneous synapses (the case of finite synaptic time constants is discussed at the end of the results section), excitatory and inhibitory conductances are given by

$$
\frac{g_{E, I}^{j}}{g_{L}^{j}}=\tau_{j} \sum_{m} a_{j m} \sum_{n} \delta\left(t-t_{m}^{n}\right) .
$$

In Eq. (2), $\tau_{j}=\mathcal{C}_{j} / g_{L}^{j}$ is the single neuron membrane time constant, $a_{j m}$ are dimensionless measures of synaptic strength between neuron $j$ and neuron $m$, $\sum_{n} \delta\left(t-t_{m}^{n}\right)$ represents the sum of all the spikes generated at times $t_{m}^{n}$ by neuron $m$. Every time the membrane potential $V_{j}$ reaches the firing threshold $\theta$, the $j$ th neuron emits a spike, its membrane potential is set to a reset $V_{r}$, and stays at that value for a refractory period $\tau_{r p}$; after this time the dynamics resumes, following Eq. (1).

We use $a_{j m}=a(a g)$ for all excitatory (inhibitory) synapses. In the homogeneous case, each neuron receives synaptic inputs from $K_{E}=K\left(K_{I}=\gamma K\right)$ excitatory (inhibitory) cells. In the network case, each neuron receives additional $K_{X}=K$ excitatory inputs from an external population firing with Poisson statistics with rate $\nu_{X}$. We use excitatory and inhibitory neurons with the same biophysical properties, hence the above assumptions imply that the firing rates of excitatory and inhibitory neurons are equal, $\nu=\nu_{E}=\nu_{I}$. Models taking into account the biophysical diversity between the excitatory and inhibitory populations are discussed in Appendix D. When heterogeneity is taken into account, the above defined values of $K_{E, I, X}$ represent the means of Gaussian distributions. We use the following single neuron parameters: $\tau_{r p}=2 \mathrm{~ms}$, $\theta=-55 \mathrm{mV}, V_{r}=-65 \mathrm{mV}, E_{E}=0 \mathrm{mV}, E_{I}=-75 \mathrm{mV}$, $E_{L}=-80 \mathrm{mV}, \tau_{j}=\tau_{L}=20 \mathrm{~ms}$. We explore various scalings of $a$ with $K$ and, in all cases, we assume that $a \ll 1$. When $a \ll 1$, an incoming spike produced by ${ }_{155}$ an excitatory presynaptic neuron produces a jump in ${ }_{156}$ the membrane potential of amplitude $a\left(E_{E}-V\right)$, where ${ }^{157}$ $V$ is the voltage just before spike arrival. In cortex, ${ }^{158}$ $V \sim-60 \mathrm{mV}$ and average amplitudes of post-synaptic ${ }_{159}$ potentials are in the order $0.5-1.0 \mathrm{mV} 3238$. Thus, 160 we expect realistic values of $a$ to be in the order of 0.01 . 23

where $\zeta$ is a white noise term, with zero mean and unit variance density, while

$$
\begin{array}{r}
\tau^{-1}=\tau_{L}^{-1}+a K\left(r_{E}+r_{I} g \gamma\right), \\
\mu=\tau\left\{E_{L} / \tau_{L}+a K\left[r_{E} E_{E}+r_{I} g \gamma E_{I}\right]\right\}, \\
\sigma^{2}(V)=a^{2} K \tau\left[r_{E}\left(V-E_{E}\right)^{2}+g^{2} \gamma r_{I}\left(V-E_{I}\right)^{2}\right] .
\end{array}
$$

In Eq. (4), $\tau$ is an effective membrane time constant, while $\mu$ and $\sigma^{2}(V)$ represent the average and the variance of the synaptic current generated by incoming spikes, respectively.

The noise term in Eq. (4) can be decomposed into an additive and a multiplicative component. The latter has an effect on membrane voltage statistics that is of the 
same order of the contribution coming from synaptic shot noise 39, a factor which has been neglected in deriving Eq. (3). Therefore, for a consistent analysis, we neglect the multiplicative component of the noise in the above derivation; this leads to an equation of the form of Eq. (4) with the substitution

$$
\sigma(V) \rightarrow \sigma(\mu) .
$$

This approach has been termed the effective time constant approximation 39. Note that the substitution of Eq. (6) greatly simplifies mathematical expressions but it is not a necessary ingredient for the results presented in this paper. In fact, all our results can be obtained without having to resort to this approximation (see Appendix A, B and D.

Current-based model. The previous definitions and results translate directly to current-based models, with the only exception that the dependency of excitatory and inhibitory synaptic currents on the membrane potential are neglected (see [10 for more details). Therefore, Eq. (1) becomes

$$
\tau_{j} \frac{d V_{j}}{d t}=-V_{j}+I_{E}^{j}-I_{I}^{j},
$$

where

$$
I_{A}^{j}=\tau_{j} \sum_{m} J_{j m} \sum_{n} \delta\left(t-t_{m}^{n}\right)
$$

represent the excitatory and inhibitory input currents. ${ }^{211}$ Starting from Eq. (7), making assumptions analogous to ${ }^{212}$ those discussed above and using the diffusion approxi- ${ }^{213}$ mation [10, the dynamics of current-based neurons is 214 given by an equation of the form of Eq. (4) with

$$
\begin{array}{r}
\tau=\tau_{L}, \quad \mu=\tau J K\left[r_{E}-g \gamma r_{I}\right], \\
\sigma^{2}=\tau J^{2} K\left[r_{E}+g^{2} \gamma r_{I}\right] ;
\end{array}
$$

Note that, unlike what happens in conductance-based 220 models, $\tau$ is a fixed parameter and does not depend on ${ }^{221}$ network firing rate or external drive. Another differ- ${ }^{222}$ ence between the current-based and conductance-based ${ }^{223}$ models is that in the latter, but not the former, model ${ }^{224}$ $\sigma$ depends on $V$; as we discussed above, this difference ${ }^{225}$ is neglected in the main text, where we use the effective ${ }^{226}$ time constant approximation.

\section{BEHAVIOR OF SINGLE NEURON RESPONSE FOR LARGE $K$}

We start our analysis investigating the effects of ${ }^{233}$ synaptic conductance on single neuron response. We ${ }^{234}$ consider a neuron receiving $K(\gamma K)$ excitatory (in- ${ }^{235}$ hibitory) inputs, each with synaptic efficacy $J(g J),{ }^{236}$ from cells firing with Poisson statistics with a rate

$$
r_{E}=\nu_{X}, \quad r_{I}=\eta \nu_{X},
$$

and analyze its membrane potential dynamics in the frameworks of current-based and conductance-based models. In both models, the membrane potential $V$ follows a stochastic differential equation of the form of Eq. (4); differences emerge in the dependency of $\tau, \mu$ and $\sigma$ on the parameters characterizing the connectivity, $K$ and $J$. In particular, in the current-based model, the different terms in Eq. (8) can be writen as

$$
\tau \sim \tau_{0}^{\text {curr }}, \quad \mu \sim K J \mu_{0}^{\text {curr }}, \quad \sigma \sim \sqrt{K} J \sigma_{0}^{\text {curr }} ;
$$

where $\tau_{0}^{\text {curr }}, \mu_{0}^{\text {curr }}$, and $\sigma_{0}^{\text {curr }}$ are independent of $J$ and $K$. In the conductance-based model, the efficacy of excitatory and inhibitory synapses depend on the membrane potential as $J=a\left(E_{E, I}-V\right)$; the different terms in Eq. (4), under the assumption that $K a \gg 1$, become of order

$$
\tau \sim \frac{\tau_{0}^{\text {cond }}}{K a}, \quad \mu \sim \mu_{0}^{\text {cond }}, \quad \sigma \sim \sqrt{a} \sigma_{0}^{\text {cond }} .
$$

Here, all these terms depend on parameters in a completely different way than in the current-based case. As we will show below, these differences drastically modify how the neural response changes as $K$ and $J$ are varied and hence the size of $J$ ensuring finite response for a given value of $K$.

The dynamics of a current-based neuron is shown in Fig. 1AAi, with parameters leading to irregular firing. Because of the chosen parameter values, the mean excitatory and inhibitory inputs approximately cancel each other, generating subthreshold average input and fluctuation-driven spikes, which leads to irregularity of firing. If all parameters are fixed while $K$ is increased $\left(J \sim K^{0}\right)$, the response changes drastically (Fig. 14Aii), since the mean input becomes much larger than threshold and firing becomes regular. To understand this effect, we analyze how terms in Eq. (4) are modified as $K$ increases. The evolution of the membrane potential in time is determined by two terms: a drift term $-(V-\mu) / \tau$, which drives the membrane potential toward its mean value $\mu$, and a noise term $\sigma / \sqrt{\tau}$, which leads to fluctuations around this mean value. Increasing $K$ modifies the equilibrium value $\mu$ of the drift force and the input noise, which increase proportionally to $K J(1-\gamma g \eta)$ and $K J^{2}\left(\gamma g^{2} \eta+1\right)$, respectively (Fig. 1B,C).

This observation suggests that, to preserve irregular firing as $K$ is increased, two ingredients are needed. First, the rates of excitatory and inhibitory inputs must be fine tuned to maintain a mean input below threshold; this can be achieved choosing $\gamma g \eta-1 \sim 1 / K J$. Second, the amplitude of input fluctuations should be preserved; this can be achieved scaling synaptic efficacy as $J \sim 1 / \sqrt{K}$. Once these two conditions are met, irregular firing is restored (Fig. 1Aiii). Importantly, in a network with $J \sim 1 / \sqrt{K}$, irregular firing emerges without fine tuning, since rates dynamically adjust to balance excitatory and inhibitory inputs and maintain mean inputs below threshold [7, 8. 
A

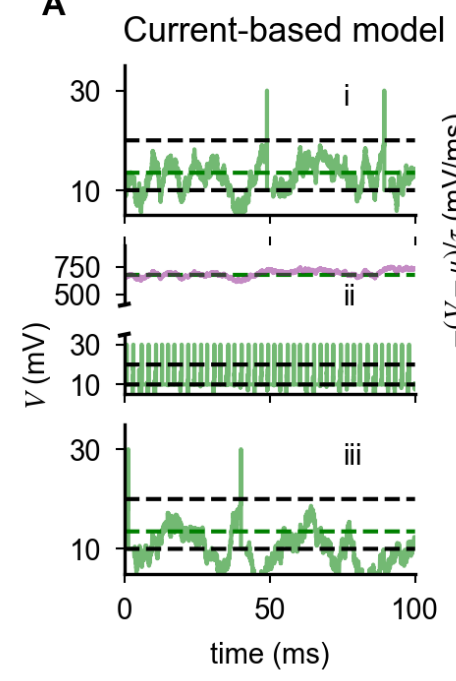

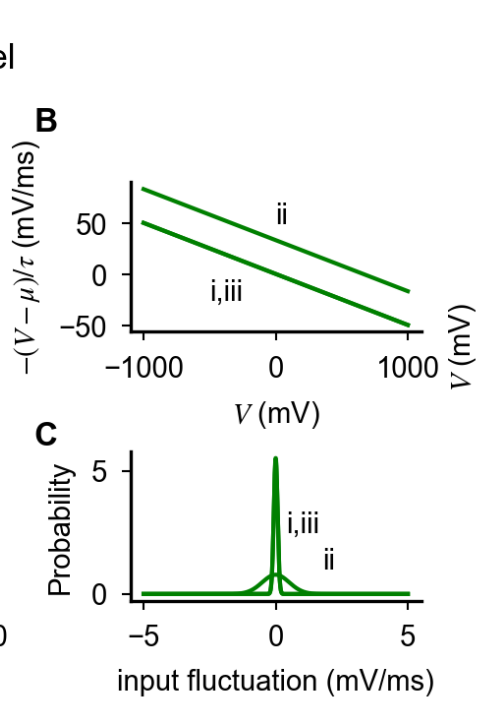

D

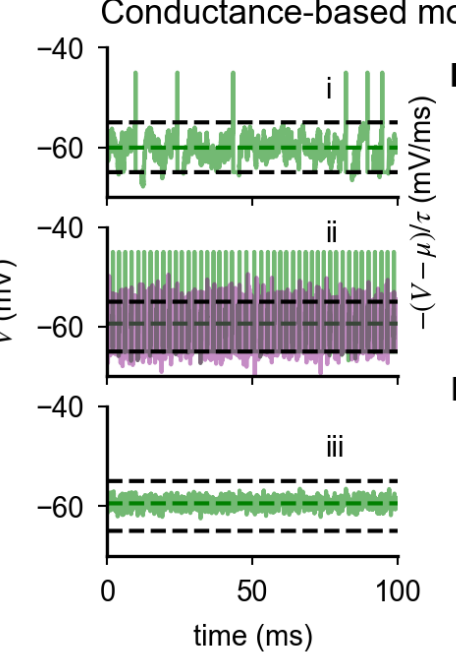

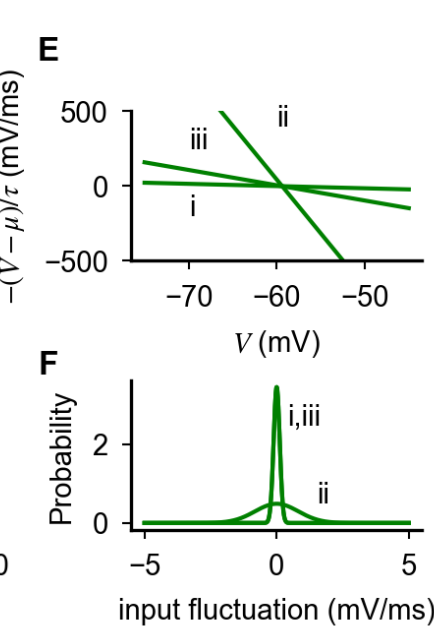

FIG. 1. Effects of coupling strength on the firing behavior of current-based and conductance-based neurons. (A) Membrane potential of a single current-based neuron for (i) $J=0.3 \mathrm{mV}, K=10^{3}, g=\gamma=1, \eta$ such that $1-g \gamma \eta=0.075$; (ii) with $K=510^{4}$; (iii) with $K=510^{4}$ and scaled synaptic efficacy $(J \sim 1 / \sqrt{K}$, which gives $J=0.04 \mathrm{mV})$ and input difference $1-g \gamma \eta=0.01 ;(\mathbf{B}, \mathbf{C})$ Effect of coupling strength on drift force and input noise in a current-based neuron. (D) Membrane potential of a single conductance-based neuron for fixed input difference $(g 1-\gamma \eta=-2.8)$ and (i) $a=0.01, K=10^{3}$; (ii) $K=510^{4}$; (iii) $K=510^{4}$ and scaled synaptic efficacy $(a \sim 1 / \sqrt{K}, a=0.001)$. (E,F) Effect of coupling strength on drift force and input noise in a conductance-based neuron. In panels $\mathbf{A}$ and $\mathbf{D}$, dashed lines represent threshold and reset (black) and equilibrium value of membrane potential (green). In panels Aii and Dii, light purple traces represent dynamics in the absence of spiking mechanism. Input fluctuations in $\mathbf{C}$ and $\mathbf{F}$ represent input noise per unit time, i.e. the integral of $\sigma \sqrt{\tau} \zeta$ of Eq. (4) computed over an interval $\Delta t$ and normalized over $\Delta t$.

We now show that this solution does not work once ${ }^{267}$ synaptic conductance is taken into account. The dy- 268 namics of a conductance-based neuron in response to the 269 inputs described above is shown in Fig. 1Di. As in the 270 current-based neuron, it features irregular firing, with 271 mean input below threshold and spiking driven by fluc- 272 tuations, and firing becomes regular for larger $K$, leaving 273 all other parameters unchanged (Fig. 1Dii). However, 274 unlike the current-based neuron, input remains below 275 threshold at large $K$; regular firing is produced by large fluctuations, which saturate response and produce spikes that are regularly spaced because of the refractory pe- ${ }^{276}$ riod. These observations can be understood looking at ${ }^{277}$ the equation for the membrane potential dynamics: in- ${ }^{278}$ creasing $K$ leaves invariant the equilibrium value of the membrane potential $\mu$ but increases the drift force and 279 the input noise amplitude as $K a$ and $\sqrt{K} a$, respectively 280 (Fig. 1E,F). Since the equilibrium membrane potential ${ }^{281}$ is fixed below threshold, response properties are deter- 282 mined by the interplay between drift force and input 283 noise, which have opposite effects on the probability of ${ }^{284}$ spike generation. The response saturation observed in Fig. 11Dii shows that, as $K$ increases at fixed $a$, fluctuations dominate over drift force. On the other hand, using the scaling $a \sim 1 / \sqrt{K}$ leaves the amplitude of fluctuations unchanged, but generates a restoring force 285 of order $\sqrt{K}$ (Fig. $1 \mathrm{E}$ ) which dominates and completely abolishes firing at strong coupling (Fig. 1Diii).

Results in Fig. 1 show that the response of a conductance-based neuron when $K$ is large depends on the balance between drift force and input noise. The scalings $a \sim O(1)$ and $a \sim 1 / \sqrt{K}$ leave one of the two contributions dominate; suggesting that an intermediate scaling could keep a balance between them. Below we derive such a scaling, showing that it preserves firing rate and CV of ISI when $K$ becomes large.

\section{A SCALING RELATION THAT PRESERVES SINGLE NEURON RESPONSE FOR LARGE $K$}

We analyze under what conditions the response of a single conductance-based neuron is preserved when $K$ is large. For a LIF neuron driven described by Eqs. 4, 5 , 6), the single cell transfer function, i.e. the dependency of the firing rate $\nu$ on the external drive $\nu_{X}$, is given by [40, 41]

$$
\nu=\left[\tau_{r p}+\tau \sqrt{\pi} \int_{v_{\min }}^{v_{\max }} d x \exp \left(x^{2}\right)(1+\operatorname{erf}(x))\right]^{-1},
$$

with

$$
v(x)=\frac{x-\mu}{\sigma}, \quad v_{\min }=v\left(V_{r}\right), \quad v_{\max }=v(\theta) .
$$


A
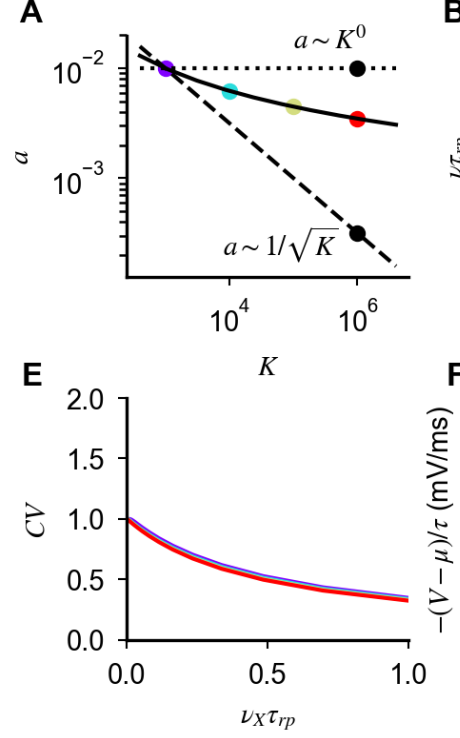

B

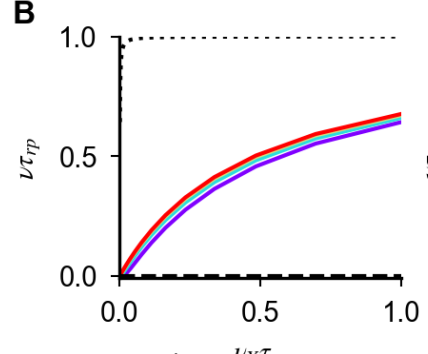

C

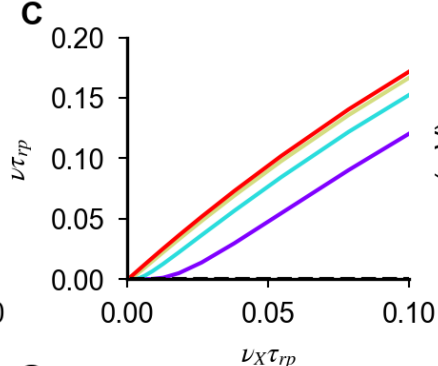

G

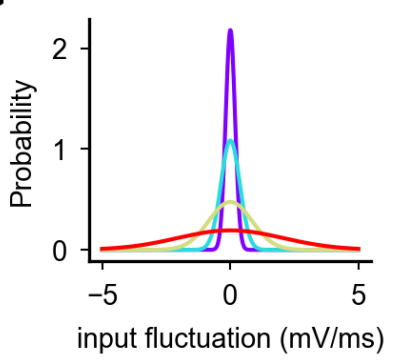

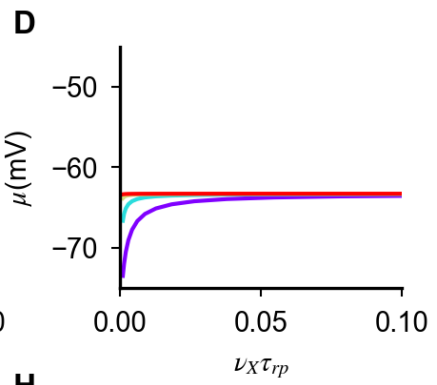

H

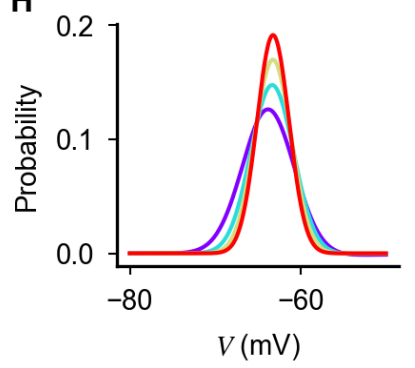

FIG. 2. The scaling of Eq. 144 preserves the response of a single conductance-based neuron for large $K$. (A) Scaling relation preserving firing in conductance-based neurons (Eq. 144, solid line); constant scaling $\left(a \sim K^{0}\right.$, dotted line) and scaling of the balanced state model $(a \sim 1 / \sqrt{K}$, dashed line) are shown as a comparison. Colored dots indicate values of $a, K$ used in the subsequent panels. (B-H) Response of conductance-based neurons, for different values of coupling strength and synaptic efficacy (colored lines). The scaling of Eq. (14) preserves how firing rate (B,C); equilibrium value of the membrane potential $(\mathbf{D})$; and $\mathrm{CV}$ of the inter-spike interval distribution $(\mathbf{E})$ depend on external input rate $\nu_{X}$. This invariance is achieved increasing the drift force $(\mathbf{F})$ and input fluctuation $(\mathbf{G})$ in a way that weakly decreases (logarithmically in $K$ ) membrane potential fluctuations $(\mathbf{H})$. Different scalings either saturate or suppress response $\left(\mathbf{B}\right.$, black lines correspond to $K=10^{5}$ and $a$ values as in panel A). Parameters: $a=0.01$ for $K=10^{3}, g=12, \eta=1.8, \gamma=1 / 4$.

In the biologically relevant case of $a \ll 1$, Eq. 10 sim- 302 plifies significantly. In fact, the distance between the equilibrium membrane potential measured in units of noise $u_{\max }$ is of order $1 / \sqrt{a}$ (except for inputs $\nu_{X} \ll$ $1 / a K \tau_{L}$, where it is of order $1 / a \sqrt{K \nu_{X} \tau_{L}} \gg 1 / \sqrt{a}$.) Therefore $u_{\max }$ is large when $a$ is small; in this limit, the firing rate is given by Kramers escape rate [42], and Eq. 10 becomes:

$$
\nu=\frac{1}{\tau_{r p}+\frac{\mathcal{Q}}{\nu_{X}}}, \quad Q=\frac{\bar{\tau} \sqrt{\pi}}{\sqrt{a} K \bar{v}} \exp \left(\frac{\bar{v}^{2}}{a}\right),
$$

where we have defined $\bar{v}^{2}=a v_{\max }^{2}$ and $\bar{\tau}=a K \nu_{X} \tau$. The motivation to introduce $\bar{v}$ and $\bar{\tau}$ is that they remain of ${ }_{312}$ order 1 in the small $a$ limit, provided the external inputs 313 $\nu_{X}$ are at least of order $1 /\left(a K \tau_{L}\right)$. When the external 314 inputs are such that $\nu_{X} \gg 1 /\left(a K \tau_{L}\right)$, these quantities 315 become independent of $\nu_{X}, a$ and $K$ and are given by

$$
\begin{array}{r}
\bar{\tau}=(1+g \gamma \eta)^{-1}, \quad \bar{v}=\frac{\theta-\bar{\mu}}{\bar{\sigma}}, \\
\bar{\mu}=\bar{\tau}\left(E_{E}+g \gamma \eta E_{I}\right), \\
\bar{\sigma}^{2}=\bar{\tau}\left[\left(\bar{\mu}-E_{E}\right)^{2}+g^{2} \gamma \eta\left(\bar{\mu}-E_{I}\right)^{2}\right] .
\end{array}
$$

The firing rate given by Eq. (12) remains finite when $a{ }_{324}$ is small and/or $K$ is large if $Q$ remains of order one; this 325 condition leads to the following scaling relationship

$$
K \sim \frac{\bar{\tau}}{\sqrt{a} \bar{v}} \exp \left(\frac{\bar{v}^{2}}{a}\right) ;
$$

303 i.e. $a$ should be of order $1 / \log (K)$.

In Appendix C, we show that expressions analogous to Eq. 12 can be derived in integrate-andfire neuron models which feature additional intrinsic voltage-dependent currents, as long as synapses are conductance-based and input noise is small $(a \ll 1)$. Examples of such models include the exponential integrateand-fire neurons with its spike-generating exponential current [43, and models with voltage-gated subthreshold currents 23. Moreover, we show that, in these models, firing remains finite if $a \sim 1 / \log (K)$, and voltagedependent currents generate corrections to the logarithmic scaling which are negligible when coupling is strong.

Since $\bar{v}$ and $\bar{\tau}$ vary with $\nu_{X}$, Eq. (14) can be satisfied, and hence firing can be supported, only if the inputs span a small range of values, such that $\bar{\tau}$ and $\bar{v}$ are approximately constant, or if $\nu_{X} \gg 1 / a K \tau_{L}$. Note that, while in the strong coupling limit (i.e. when $K$ goes infinity), only the second of these two possibilities can be implemented with input rates spanning physiologically relevant values, both are are admissible when coupling is moderate (i.e. when $K$ is large but finite, a condition consistent with experimental data on cortical net- 
works [44, 45]). In what follows, with the exception of 377 the section on finite synaptic time constant, we focus on 378 the case $\nu_{X} \gg 1 / a K \tau_{L}$, and investigate how different 379 properties evolve with $K$ using the scaling defined by 380 Eq. (14) with $\bar{v}$ and $\bar{\tau}$ given by Eq. (13). Importantly, 381 all the results discussed below hold for inputs outside 382 the region $\nu_{X} \gg 1 / a K \tau_{L}$, as long as $\nu_{X}$ is at least of or- 383 der $1 / a K \tau_{L}$ (a necessary condition for the derivation of Eq. (12) to be valid), and that inputs span a region small enough for the variations of $\bar{v}$ and $\bar{\tau}$ to be negligible.

In Fig. $2 \mathrm{~A}$, we compare the scaling defined by Eq. 14 with the $a \sim 1 / \sqrt{K}$ scaling of current-based neurons. 384 At low values of $K$, the values of $a$ obtained with the 385 two scalings are similar; at larger values of $K$, synaptic 386 strength defined by Eq. (14) decays as $a \sim 1 / \log (K)$, i.e. ${ }^{387}$ synapses are stronger in the conductance-based model than in the current-based model. Examples of single neuron transfer function computed from Eq. 10 for different coupling strength are shown in Fig. 2B,C. Responses are nonlinear at onset and close to saturation. 388 As predicted by the theory, scaling $a$ with $K$ according 389 to Eq. (14) preserves the firing rate over a region of in- 390 puts that increases with coupling strength (Fig. 2 C,D), 391 while the average membrane potential remains below 392 threshold (Fig. 2D). The quantity $\bar{v} / \sqrt{a}$ represents the distance from threshold of the equilibrium membrane potential in units of input fluctuations; Eq. (14) implies that this distance increases with coupling strength. When $K$ is very large, the effective membrane time constant, which is of order $\tau \sim 1 / a K \nu_{X}$, becomes small and firing is driven by fluctuations that, on the time scale of 396 this effective membrane time constant, are rare.

We next investigated if the above scaling preserves irregular firing by analyzing the $\mathrm{CV}$ of interspike intervals. This quantity is given by 10

$$
C V^{2}=2 \pi \nu^{2} \tau^{2} \int_{v_{\min }}^{v_{\max }} d x e^{x^{2}} \int_{-\infty}^{x} d y e^{y^{2}}(1+\operatorname{erf}(y))^{2}
$$

and, for the biologically relevant case of $a \ll 1$ and $\mu<$ $\theta$, reduces to (see Appendix B for details)

$$
C V=1-\tau_{r p} \nu
$$

i.e. the $\mathrm{CV}$ is close to one at low rates and it decays monotonically as the neuron approaches saturation. Critically, Eq. (16) depends on coupling strength only through $\nu$, hence any scaling relation preserving firing rate will also produce $\mathrm{CV}$ of order one at low rate. We validated numerically this result in Fig. $2 \mathrm{E}$.

We now investigate how Eq. (14) preserves irregular firing in conductance-based neurons. We have shown that increasing $K$ at fixed $a$ produces large input and membrane fluctuations, which saturate firing; the scaling $a \sim 1 / \sqrt{K}$ preserves input fluctuations but, because of the strong drift force, suppresses membrane potential fluctuations, and hence firing. The scaling of Eq. (14), at every value of $K$, yields the value of $a$ that balances the contribution of drift and input fluctuations, so that membrane fluctuations are of the right size to preserve the rate of threshold crossing. Note that, unlike what happens in the current-based model, both input fluctuations and drift force increase with $K$ (Fig. $2 \mathrm{~F}, \mathrm{G}$ ) while the membrane potential distribution, which is given by [46]

$$
P(V)=\frac{2 \nu \tau}{\sigma} \int_{v(V)}^{v_{\max }} d x \theta\left(x-v\left(V_{r}\right)\right) \exp \left[x^{2}-v(V)^{2}\right],
$$

slowly becomes narrower (Fig. $2 \mathrm{H}$ ). This result can be understood by noticing that, when $a \ll 1$ and neglecting the contribution due to the refractory period, Eq. (17) reduces to

$$
P(V)=\frac{1}{\sigma \sqrt{\pi}} \exp \left(-\frac{(V-\mu)^{2}}{\sigma^{2}}\right) .
$$

Hence, the probability distribution becomes Gaussian when coupling is strong, with a variance proportional to $\sigma^{2} \sim a$. We note that, since $a$ is of order $1 / \log K$, the width of the distribution becomes small only for unrealistically large values of $K$.

\section{3 \\ V. ASYNCHRONOUS IRREGULAR ACTIVITY IN NETWORK RESPONSE AT STRONG COUPLING}

We have so far considered the case of a single neuron subjected to stochastic inputs. We now show how the above results generalize to the network case, where inputs to a neuron are produced by a combination of external and recurrent inputs.

We consider networks of recurrently connected excitatory and inhibitory neurons, firing at rate $\nu$, stimulated by an external population firing with Poisson statistics with firing rate $\nu_{X}$. Using again the diffusion approximation, the response of a single neuron in the networks is given by Eq. 10 (and hence Eq. 12) with

$$
r_{E}=\nu_{X}+\nu, \quad r_{I}=\nu .
$$

Eq 10, if all neurons in a given population are described by the same single cell parameters and the network is in an asynchronous state in which cells fire at a constant firing rate, provides an implicit equation whose solution is the network transfer function. Example solutions are shown in Fig. $3 \mathrm{~B}$ (numerical validation of the mean field results is provided in Appendix E. In Appendix $\mathrm{D}$, we prove that firing in the network is preserved when coupling is strong if parameters are rescaled according to Eq. (14). Moreover, we show that response nonlinearities are suppressed and the network response in the strong coupling limit (i.e. when $K$ goes infinity) is given, up to saturation, by

$$
\nu=\rho \nu_{X} .
$$


A

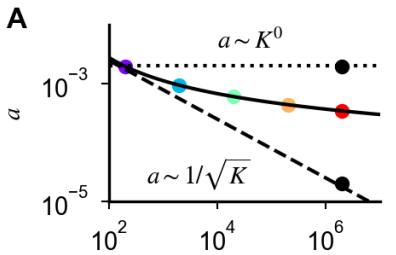

C

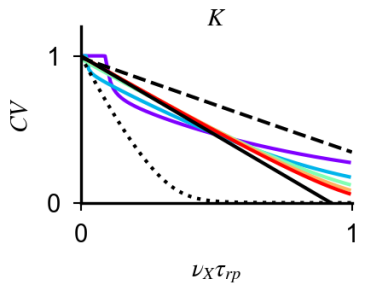

B

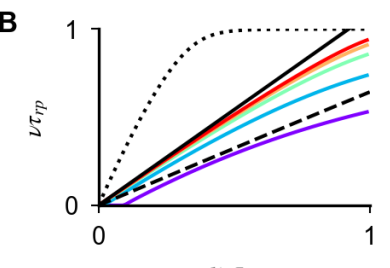

D

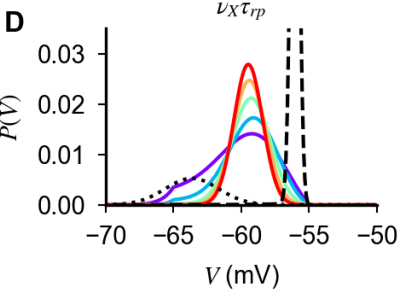

FIG. 3. Response of networks of conductancebased neurons for large $K$. (A) Scaling relation defined by self-consistency condition given by Eqs . (14) and 19) (black line), values of parameters used in panels B-D (colored-dots). Constant scaling ( $a \sim K^{0}$, dotted line) and scaling of the balanced state model $(a \sim 1 / \sqrt{K}$, dashed line) are shown for comparison. $(\mathbf{B}, \mathbf{C})$ Firing rate and $\mathrm{CV}$ of ISI as a function of external input, obtained from Eqs. 10 and 15 (colored lines) with strong coupling limit solution of 466 Eqs. 200 and (16) (black line). (D) Probability distribution 467 of the membrane potential obtained from (17). In panels ${ }_{468}$ B-D, dotted and dashed lines represent quantities obtained with the scalings $J \sim K^{0}$ and $J \sim 1 / \sqrt{K}$, respectively, for values of $K$ and $J$ indicated in panel A (black dots). Parameters: $\gamma=1 / 4$ and $g=30$.

The parameter $\rho$, which is obtained solving Eq. (12) self-consistently (see Appendix D for details), is the response gain in the strong coupling limit. Finally, our 475 derivation implies that Eq. (14) preserves irregular fir- ${ }_{476}$ ing and creates a probability distribution of membrane ${ }^{477}$ potential whose width decreases only logarithmically as $K$ increases (Fig. $3 \mathrm{C}$,D and numerical validation in Appendix E), as in the single neuron case. While this logarithmic decrease is a qualitative difference with the current-based balanced state in which the width stays finite in the large $K$ limit, in practice for realistic values of $K$, realistic fluctuations of membrane potential (a few $\mathrm{mV}$ ) can be observed in both cases.

We now turn to the question of what happens in net- ${ }^{486}$ works with different scalings between $a$ and $K$. Our ${ }_{487}$ analysis of single neuron response described above shows ${ }_{488}$ that scalings different from that of Eq. (14) fail to pre- ${ }_{489}$ serve firing for large $K$, as they let either input noise 490 or drift dominate. However, the situation in networks might be different, since recurrent interactions could in ${ }_{492}$ principle adjust the statistics of input currents such that ${ }_{493}$ irregular firing at low rates is preserved when coupling 494 becomes strong. Thus, we turn to the analysis of the net- 495 work behavior when a scaling $a \sim K^{-\alpha}$ is assumed. For 496 $\alpha \leq 0$, the dominant contribution of input noise at the ${ }_{497}$ single neuron level (Figs. 1 and 2 ) generates saturation of response and regular firing in the network (Fig. 3). This can be understood by noticing that, for large $K$, the factor $Q$ in Eq. 12 becomes negligible and the selfconsistency condition defining the network rate is solved by $\nu=1 / \tau_{r p}$. For $\alpha>0$, the network response for large $K$ is determined by two competing elements. On the one hand, input drift dominates and tends to suppress firing (Figs. 1 and 2). On the other hand, for the network to be stable, inhibition must dominate recurrent interactions 9. Hence, any suppression in network activity reduces recurrent inhibition and tends to increase neural activity. When these two elements conspire to generate a finite network response, the factor $\mathcal{Q}$ in Eq. 12 must be of order one and $\bar{v} \sim a \sim K^{-\alpha}$. In this scenario, the network activity exhibits the following features (Fig. 33): (i) the mean inputs drive neurons very close to threshold $\left(\theta-\bar{\mu} \sim a \bar{\sigma} \sim K^{-\alpha}\right)$; (ii) the response of the network to external inputs is linear and, up to corrections of order $K^{-\alpha}$, given by

$$
\nu=\frac{\left(E_{E}-\theta\right) \nu_{X}}{\theta(1+g \gamma)-E_{E}-g \gamma E_{I}} ;
$$

(iii) firing is irregular (because of Eq. (16p); (iv) the width of the membrane potential distribution is of order $a \sim K^{-\alpha}$ (because of Eq. (18)). Therefore, scalings different from that of Eq. (14) can produce asynchronous irregular activity in networks of conductance-based neurons, but this leads to networks with membrane potentials narrowly distributed close to threshold, a property which seems at odds with what is observed in cortex 47 52 .

\section{ROBUST LOGNORMAL DISTRIBUTION OF FIRING RATES IN NETWORKS WITH HETEROGENEOUS CONNECTIVITY}

Up to this point, we have assumed a number of connections equal for all neurons. In real networks, however, this number fluctuates from cell to cell. The goal of this section is to analyze the effects of heterogeneous connectivity in networks of conductance-based neurons.

We investigated numerically the effects of connection heterogeneity as follows. We chose a Gaussian distribution of the number of connections per neuron, with mean $K$ and variance $\Delta K^{2}$ for excitatory connections, and mean $\gamma K$ and variance $\gamma^{2} \Delta K^{2}$ for inhibitory connections. The connectivity matrix was constructed by drawing first randomly $\mathrm{E}$ and I in-degrees $K_{E, X, I}^{i}$ from these Gaussian distributions for each neuron, and then selecting at random $K_{E, X, I}^{i}$ E/I pre-synaptic neurons. We then simulated network dynamics and measured the distribution of rates and CV of the ISI in the population. Results for different values of $C V_{K}=\Delta K / K$ are shown in Fig. $4 \mathrm{~A}-\mathrm{C}$. For small and moderate values of connection heterogeneity, increasing $C V_{K}$ broadens the 

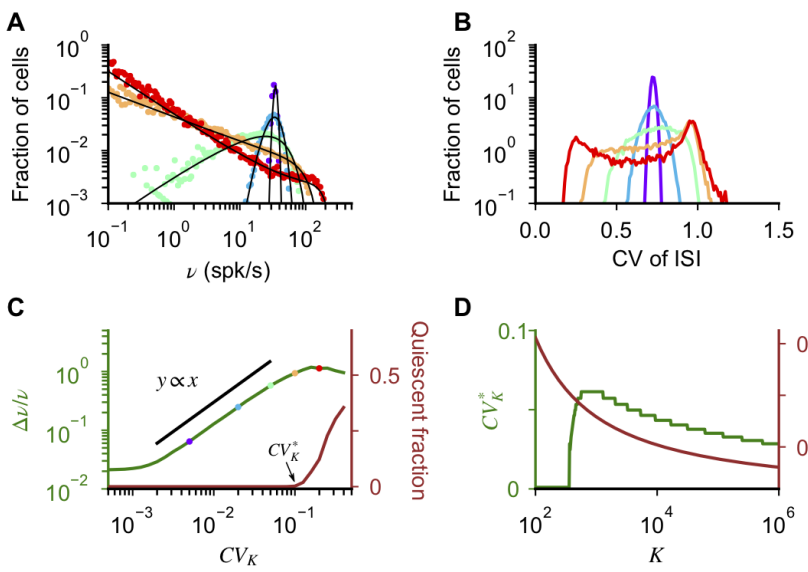

D

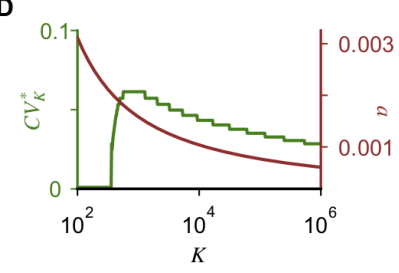

FIG. 4. Effects of heterogeneous connectivity on the 528 network response. (A-B) Distribution of $\nu$ and $C V$ of ${ }_{529}$ ISI computed from network simulations (dots) and from the 530 mean field analysis (A, black lines) for different values of ${ }_{531}$ $C V_{K}$ (values are indicated by dots in panel $\mathbf{C}$ ). (C) $\Delta \nu / \nu$ (green, left axis) and fraction of quiescent cells (brown, right axis) computed from network simulations as a function of $C V_{K}$. For $C V_{K} \lesssim C V_{K}^{*}, \Delta \nu / \nu$ increases linearly, as predicted by the mean field analysis; deviations from linear scaling emerge for $C V_{K} \gtrsim C V_{K}^{*}$, when a significant fraction of cells become quiescent. The deviation from linear scaling at low $C V_{K}$ is due to sampling error in estimating 538 the firing rate from simulations. (D) $C V_{K}^{*}$ as a function of 539 $K$ computed from the mean field theory (green, left axis), 540 with $a$ rescaled according to Eq. (14). For large $K, C V_{K}^{*}$ decays proportionally to $a$ (brown, right axis). When $K$ is too low, the network is silent and $C V_{K}^{*}=0$. In panels A-C $K=10^{3}, g=20, a=1.610^{-3}, N_{E}=N_{X}=N_{I} / \gamma=10 K$, $\nu_{X}=0.05 / \tau_{r p}$. In network simulations, the dynamics was run for 20 seconds using a time step of $50 \mu$ s Parameters in panel $\mathbf{D}$ are as in Fig. 3 .
526

.

distribution of rates and CV of the ISI, but both dis- ${ }^{548}$ tributions remain peaked around a mean rate that is ${ }^{549}$ close to that of homogeneous networks (Fig. 4A,B). For 550 larger $C V_{K}$, on the other hand, the distribution of rates ${ }^{551}$ changes its shape, with a large fraction of neurons mov- ${ }^{552}$ ing to very low rates, while others increase their rates 553 (Fig. 4 A) and the distribution of the CV of ISI becomes ${ }^{554}$ bimodal, with a peak at low CV corresponding to the ${ }^{555}$ high rate neurons, while the peak at a CV close to 1 cor- 556 responds to neurons with very low firing rates (Fig. $4 \mathrm{~B}$ ). ${ }^{557}$

To characterize more systematically the change in the ${ }^{558}$ distribution of rates with $C V_{K}$, we measured, for each ${ }^{559}$ value of $C V_{K}$, the fraction of quiescent cells, defined as 560 the number of cells that did not spike during 20 seconds 561 of the simulated dynamics (Fig. $4 \mathrm{C}$ ). This analysis shows 562 that the number of quiescent cells, and hence the distri- 563 bution of rates, changes abruptly as the $C V_{K}$ is above 564 a critical value $C V_{K}^{*}$. Importantly, unlike our definition 565 of the fraction of quiescent cells, this abrupt change is 566 a property of the network that is independent of the ${ }_{567}$ duration of the simulation.

To understand these numerical results, we performed ${ }_{569}$ a mean field analysis of the effects of connection heterogeneity on the distribution of rates (Appendix F). This analysis captures quantitatively numerical simulations (Fig. 4A) and shows that, in the limit of small $C V_{K}$ and $a$, rates in the network are given by

$$
\nu_{i}=\nu_{0} \exp \left[\Omega \frac{C V_{K}}{a} z_{i}\right]
$$

where $\nu_{0}$ is the population average in the absence of heterogeneity, $z_{i}$ is a Gaussian random variable, and the prefactor $\Omega$ is independent of $a, K$ and $\nu_{X}$. The exponent in Eq. 22 represents a quenched disorder in the value of $v^{i}$, i.e. in the distance from threshold of the single cell $\mu^{i}$ in units of input noise. As shown in Appendix F. Eq. 22 implies that the distribution of rates is lognormal, a feature consistent with experimental observations 53 55] and distributions of rates in networks of current-based LIF neurons 56. It also implies that the variance of the distribution $\Delta \nu / \nu$ should increase linearly with $C V_{K}$, a prediction which is confirmed by numerical simulations (Fig. 4 C). The derivation in Appendix $\mathrm{F}$ also provides an explanation for the change in the shape of the distribution for larger $C V_{K}$. In fact, for larger heterogeneity, the small $C V_{K}$ approximation is not valid and fluctuations in input connectivity produce cells for which $\mu^{i}$ far from $\theta$, that are either firing at extremely low rate $\left(\mu^{i}<\theta\right)$ or regularly $\left(\mu^{i}>\theta\right)$. The latter generates the peak at low values in the CV of the ISI seen for large values $C V_{K}$.

The quantity $C V_{K}^{*}$ represents the level of connection heterogeneity above which significant deviations from the asynchronous irregular state emerges, i.e. large fractions of neurons show extremely low or regular firing. Eq. (22) suggests that $C V_{K}^{*}$ should increase linearly with $a$. We validated this prediction with our mean field model, by computing the minimal value of $C V_{K}$ at which $1 \%$ of the cells fire at rate of $10^{-3} \mathrm{spk} / \mathrm{s}$. (Fig. 4D). Note that the derivation of Eq. (22) only assumes $a$ to be small and does not depend on the scaling relation between $a$ and $K$. On the other hand, the fact that $C V_{K}^{*}$ increases linearly with $a$ makes the state emerging in networks of conductance-based neurons with $a \sim 1 / \log (K)$ significantly more robust to connection fluctuations than that emerging with $a \sim$ $K^{-\alpha}$, for which $C V_{K}^{*} \sim K^{-\alpha}$, and with current-based neurons, where $C V_{K}^{*} \sim 1 / \sqrt{K}$ [57. Note that, while in randomly connected networks $C V_{K} \sim 1 / \sqrt{K}$, a larger degree of heterogeneity has been observed in cortical networks [51, 57,61]. Our results show that networks of conductance-based neurons could potentially be much more robust to such heterogeneities than networks of current-based neurons. 


\section{COMPARISON WITH EXPERIMENTAL DATA}

The relation between synaptic efficacy and number of connections per neuron has been recently studied experimentally using a culture preparation [62]. In this paper, it was found that cultures in which $K$ was larger had weaker synapses than cultures with smaller $K$ (Fig. 5). In what follows we compare this data with the scalings expected in networks of current-based and conductancebased neurons, and discuss implications for in vivo networks.

In the current-based model, the strength of excitatory and inhibitory post synaptic potentials as a function of $K$ can be written as $J_{E}=J_{0} / \sqrt{K}$ and $J_{I}=g J_{E}$. In the conductance-based model, these quantities become $J_{E}=\left(V-E_{E}\right) a$ and $J_{I}=g\left(V-E_{I}\right) a$; where $a=a(K, \bar{v})$ is given by Eq. (14) while, for the dataset of 62, $V \sim-60 \mathrm{mV}, J_{E} \sim J_{I}, E_{E} \sim 0 \mathrm{mV}$ and $E_{I} \sim-80 \mathrm{mV}$. For each model, we inferred free parameters from the data with a least-squares optimization in logarithmic scale (best fit: $g=1.1$ and $J_{0}=20 \mathrm{mV}$ in the current-based model; $g=3.4$ and $\bar{v}=0.08$ in the conductance-based model) and computed the expected synaptic strength as a function of $K$ (lines in Fig. 5 A). Our analysis shows that the performance of the currentbased and the conductance-based model in describing the data, over the range of $K$ explored in the experiment, are similar, with the former being slightly better than the latter (root mean square $2.2 \mathrm{mV}$ vs $2.4 \mathrm{mV}$ ). This result is consistent with the observation made in 62 that, when fitted with a power-law $J \sim K^{-\beta}$, data are best described by $\beta=0.59$ but are compatible with a broad range of values (95\% confidence interval: [0.47:0.70]). Note that even though both models give similar results for PSP amplitudes in the range of values of $K$ present in cultures $(\sim 50-1000)$, they give significantly different predictions for larger values of $K$. For instance, for $K=10,000, J_{E}$ is expected to be $\sim 0.2 \mathrm{mV}$ in the current-based model and $\sim 0.7 \mathrm{mV}$ in the conductancebased model.

In Fig. 5B, we plot the distance between the equilibrium membrane potential $\mu$ and threshold $\theta$ in units of input fluctuations, $\bar{v} / \sqrt{a}$ as a function of $K$ using the value of $\bar{v}$ obtained above, and find that the expected value in vivo, where $K \sim 10^{3}-10^{4}$, is in the range 2-3. In Fig. 5 C,D, we plot how total synaptic excitatory conductance, and effective membrane time constant, change as a function of $K$. Both quantities change significantly faster using the conductance-based scaling $\left(g_{E} / g_{L} \sim K / \log (K) ; \tau / \tau_{L} \sim \log (K) / K\right)$ than what expected by the scaling of the current-based model $\left(g_{E} / g_{L} \sim \sqrt{K} ; \tau / \tau_{L} \sim 1 / \sqrt{K}\right)$. For $K$ in the range $10^{3}-10^{4}$ and mean firing rates in the range $1-5 \mathrm{spk} / \mathrm{s}$, the total synaptic conductance is found to be in a range from about 2 to 50 times the leak conductance, while the effective membrane time constant is found to be smaller than the membrane time constant by a factor 2 to 50 .
628 629
We compare these values with available experimental data in the Discussion.

\section{EFFECT OF FINITE SYNAPTIC TIME CONSTANTS}

Results shown in Fig. 5 beg the question whether the assumption of negligible synaptic time constants we have made in our analysis is reasonable. In fact, synaptic decay time constants of experimentally recorded postsynaptic currents range from a few ms (for AMPA and $\mathrm{GABA}_{A}$ receptor-mediated currents) to tens of ms (for $\mathrm{GABA}_{B}$ and NMDA receptor-mediated currents, see e.g. 63]), i.e. they are comparable to the membrane time constant already at weak coupling, where $\tau \sim \tau_{L}$ is typically in the range $10-30 \mathrm{~ms} 64,65$. In the strong coupling limit, the effective membrane time constant goes to zero, and so this assumption clearly breaks down. In this section, we investigate the range of validity of this assumption, and what happens once the assumption of negligible time constants is no longer valid.

With finite synaptic time constants, the temporal evolution of conductances of Eq. (2) is replaced by

$$
\tau_{E, I} \frac{d g_{E, I}^{j}}{d t}=-g_{E, I}^{j}+g_{L}^{j} \tau_{E, I} \sum_{m} a_{j m} \sum_{n} \delta\left(t-t_{m}^{n}\right) .
$$

It follows that the single-neuron membrane potential dynamics is described by Eqs. (123). Here, for simplicity, we take excitatory and inhibitory synaptic currents to have the same decay time constant $\tau_{S}$. Fig. 6A shows how increasing the synaptic time constant modifies the mean firing rate of single integrate-and-fire neurons in response to $K(\gamma K)$ excitatory (inhibitory) inputs with synaptic strength $a(g a)$ and frequency $\nu_{X}\left(\eta \nu_{X}\right)$. The figure shows that, though the mean firing rate is close to predictions obtained with instantaneous synapses for small $\tau_{S} / \tau$, deviations emerge for $\tau_{S} / \tau \sim 1$, and firing is strongly suppressed as $\tau_{S} / \tau$ becomes larger. To understand these numerical results, we resort again to the diffusion approximation 67, together with the effective time constant approximation [11, 68, to derive a simplified expression of the single neuron membrane potential dynamics with finite synaptic time constant (details in Appendix G]; this is given by

$$
\tau \frac{d V}{d t}=-(V-\mu)+\sigma \sqrt{\frac{\tau}{\tau_{S}}} z,
$$

where $\tau, \mu$ and $\sigma$ are as in the case of negligible synaptic time constant (Eq. (5)), whilst $z$ is an OrnsteinUhlenbeck process with correlation time $\tau_{S}$. It follows that, with respect to Eq. (4), input fluctuations with frequency larger than $1 / \tau_{S}$ are suppressed and, for large $\tau_{S} / \tau$, the membrane potential dynamics is given by

$$
V(t)=\mu+\sigma \sqrt{\frac{\tau}{\tau_{S}}} z(t),
$$



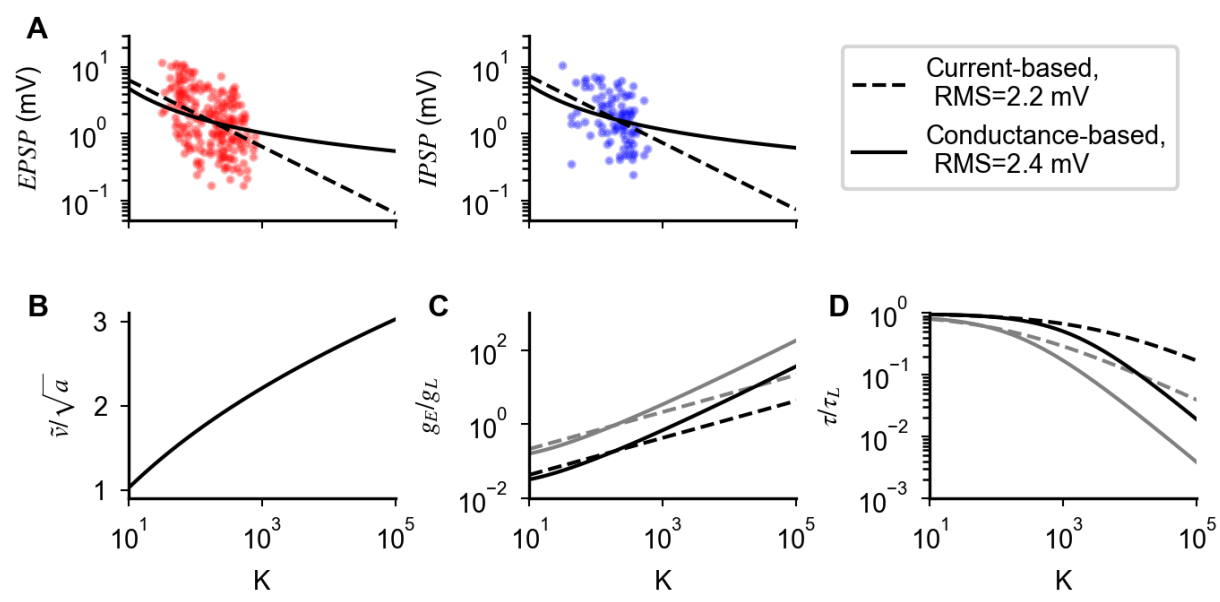

FIG. 5. Comparison of predictions given by current-based and the conductance-based models in describing experimental data from cultures. A Strength of excitatory (EPSP) and inhibitory (IPSP) post synaptic potentials recorded in 62. are compared with best fits using scaling relationships derived from networks with current-based synapses (dashed line) and conductance-based synapses (continuous line). Root mean square (RMS) and best fit parameters are: RMS $=2.2 \mathrm{mV}$, $g=1.1, J_{0}=20 \mathrm{mV}$, for the current-based model; and RMS $=2.4 \mathrm{mV}, g=3.4, \bar{v}=0.08$, for the conductance-based model. B Value of $\bar{v} / \sqrt{a}$ predicted by the conductance-based model as a function of $K$. C Ratio between excitatory and leak conductance as a function of $K$, for $\nu_{E}=\nu_{I}=\nu_{X}=1 \mathrm{spk} / \mathrm{s}$ (black) and $\nu_{E}=\nu_{I}=\nu_{X}=5 \mathrm{spk} / \mathrm{s}$ (gray) obtained with $a$ rescaled as Eq. 14 (continuous line) and as $1 / \sqrt{K}$ (dashed line). D Ratio between $\tau$ and $\tau_{L}$ as a function of $K$, parameters and scaling as in panel $\mathbf{C}$.

i.e. the membrane potential is essentially slaved to a time 704 dependent effective reversal potential corresponding to 705 the r.h.s. of Eq. 25) (14). Note that Eq. 25) is valid only 706 in the subthreshold regime. When the r.h.s. of Eq. 25) 707 exceeds the threshold, the neuron fires a burst of action 708 potentials whose frequency, in the strong coupling limit, 709 is close to the inverse of the refractory period 69. As 710 $\nu_{X}$ increases, the equilibrium value $\mu$ remains constant 711 while $\tau$ decreases, leading to a suppression of membrane 712 fluctuations (Fig. 6D), and in turn to the suppression of 713 response observed in Fig. 6A.

In Appendix G, we use existing analytical expansions 67, 69, 70], as well as numerical simulations, to shows that neural responses obtained with finite $\tau_{S}$ are in good agreement with predictions obtained with instantaneous synapses as long as $\tau_{S} / \tau \lesssim 0.1$. It follows that the single neuron properties we discussed in the case of instantaneous synapses hold in the region of inputs for which $\tau_{S} / \tau \lesssim 0.1$ (i.e. $\nu_{X} \lesssim 0.1 / a K \tau_{S}$ ) and the derivation of Eq. (14) is valid (i.e. $\nu_{X}$ is at least of order $\left.1 / a K \tau_{L}\right)$. Thus, there is at best a narrow range of inputs for which these properties carry over to the finite synaptic constant case. Interestingly, when biolog- 726 ically relevant parameters are considered (e.g. Fig. 6), 727 inputs within this region generate firing rates that are ${ }_{728}$ in the experimentally observed range in cortical net- ${ }_{729}$ works [23, 47 55. The analysis of Appendix G also 730 shows that, when $\tau_{S} / \tau \sim 1$, i.e. once the input rate ${ }_{731}$ $\nu_{X}$ is of order $1 / a K \tau_{S}$, firing is suppressed exponen- 732 tially. The scaling relation of Eq. (14) does not pre- ${ }_{733}$ vent this suppression, which emerges for external rates 734 of order $1 / a K \tau_{S} \sim \log (K) / K \tau_{S}$. Scalings of the form $a \sim K^{-\alpha}$, with $\alpha>0$, on the other hand, create a larger region of inputs for which $\tau_{S} / \tau \ll 1$ but, as we showed when studying the neural dynamics with instantaneous synapses, fail in generating response for large $K$. We next asked if another scaling relation between $a$ and $K$ could prevent suppression of neural response when $\tau_{S}$ is finite. The single neuron response computed in Appendix $\mathrm{G}$ is a nonlinear function of the input $\nu_{X}$, which depends parametrically on $a$ and $K$. It follows that, in order to preserve the single neuron response, $a$ should scale differently with $K$ for different values of $\nu_{X}$. Since in cortical networks input rates, i.e. $\nu_{X}$, change dynamically on a time scale much shorter than that over which plasticity can modify synaptic connections, we conclude that a biologically realistic scaling between $a$ and $K$, which prevents suppression of neural response when $\tau_{S}$ is finite in a broad range of external inputs, does not exist. Moreover, the membrane potential dynamics for large $K$ and $\tau_{S} / \tau$ (Eq. (25p) becomes independent of $a$. This shows that rescaling synaptic efficacy with $K$ cannot prevent suppression of response.

We next examined the effect of synaptic time constant on network response. Numerically computed responses in networks of neurons with finite synaptic time constant are shown in Fig. 6B,C. Network response is close to the prediction obtained with instantaneous synapses for small $\tau_{S} / \tau$, and deviations emerge for $\tau_{S} / \tau \sim 1$. Hence, analogously to the single neuron case, the network properties discussed in the case of instantaneous synapses remain valid for low inputs. However, unlike 

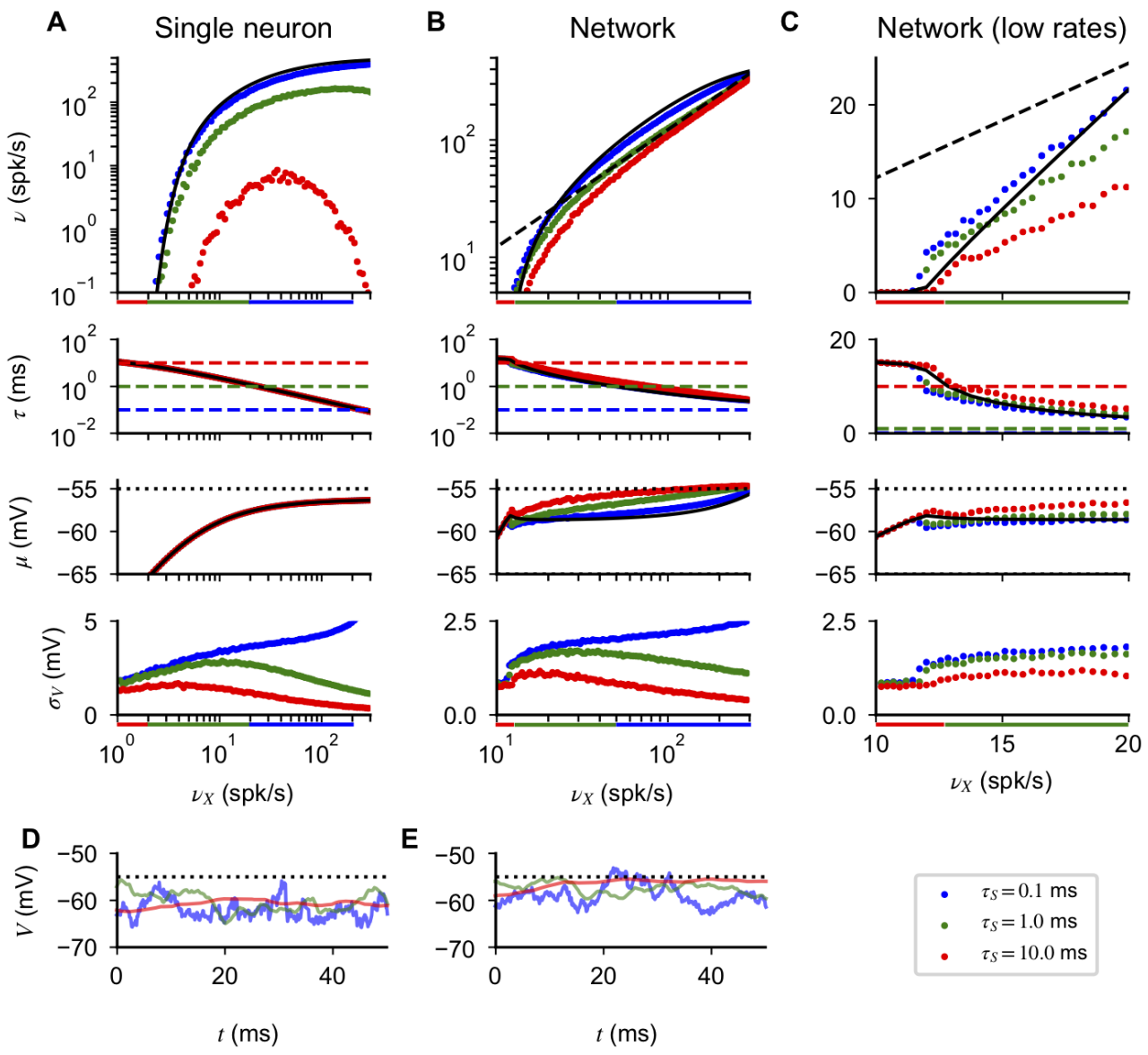

$t(\mathrm{~ms})$

FIG. 6. Effects of synaptic time constant on single neuron and network response. (A) Single neuron response as a function of input rate $\nu_{X}$, computed numerically from Eqs. 11, 23) for different synaptic time constants $\tau_{S}$ (indicated in the bottom right of the figure). In all panels, black lines correspond to the prediction obtained with instantaneous synapses. Colored bars below the first and the fourth row indicate inputs that gives $0.1<\tau_{S} / \tau<1$, i.e. the region where deviations in the neural response from the case of instantaneous synapses emerge. Firing rates (first row) match predictions obtained for instantaneous synapses for small $\tau_{S} / \tau$; significant deviations and response suppression emerge for larger $\tau_{S} / \tau$. The effective membrane time constant $\left(\tau\right.$, second row) decreases with input rate, is independent of $\tau_{S}$, and reaches the value $\tau_{S} / \tau \sim 1$ for different levels of external drive (dashed lines represent the different values of $\tau_{S}$ ). The equilibrium value of the membrane potential ( $\mu$, third row) increases with input rate and is independent of $\tau_{S}$ (dotted line represents spiking threshold). The fluctuation of the membrane potential ( $\sigma_{V}$, fourth row) has a non-monotonic relationship with input rate, and peaks at a value of $\nu_{X}$ for which $\tau$ is of the same order as $\tau_{S}$. (B) Analogous to panel $\mathbf{A}$ but in the network case. Firing rates are no longer suppressed as $\tau_{S} / \tau$ increases, but approach the response scaling predicted by Eq. 21. (dashed line). As discussed in the text, high firing rates are obtained by increasing the value of $\mu$ towards threshold. (C) Zoom of panel $\mathbf{B}$ in the neurobiologically relevant region of low rates. (D, E) Examples of membrane potential dynamics for single neuron $(\mathbf{D})$ and network $(\mathbf{E})$ in the absence of spiking mechanisms $\left(\nu_{X}=5 \mathrm{spk} / \mathrm{s}\right.$ in $\mathbf{D}$ and $20 \mathrm{spk} / \mathrm{s}$ in $\left.\mathbf{E}\right)$. High frequency fluctuations are suppressed at large $\tau_{S}$. In the network case, increasing $\tau_{S}$ reduces recurrent inhibition and produces membrane potential trajectories which are increasingly closer to firing threshold. Single neuron parameters: $a=0.01, K=10^{3}, g=8, \eta=1.5, \gamma=1 / 4$. Network parameters: $a=0.0016, K=10^{3}, g=20, \gamma=1 / 4$. Simulations were performed with the simulator BRIAN2 [66], with neurons receiving inputs from $N_{E X, I X}=10 \mathrm{~K}$ independent Poisson units firing at rates $\nu_{X}, \eta \nu_{X}$, in the single neuron case, or $\nu_{X}$, in the network case. Network simulations used $N_{E, I}=10 \mathrm{~K}$ excitatory and inhibitory neurons.

the single neuron case, no suppression appears for larger 742 $\tau_{S} / \tau$. This lack of suppression in the network response, ${ }^{743}$ analogously to the one we discussed in networks with in- 744 stantaneous synapses and $a \sim K^{-\alpha}$, is a consequence of ${ }_{745}$ the fact that, to have stable dynamics when $K$ is large, 746 inhibition must dominate recurrent interactions 9. In 747 this regime, any change which would produce suppres- 748 sion of single neuron response (e.g. increase of $\nu_{X}$ or $\tau_{S}$ ) lowers recurrent inhibition and increases the equilibrium value of the membrane potential $\mu$ (Fig. 6B,C,E). The balance between these two effects determines the network firing rate and, when $\tau_{S} / \tau \gg 1$, generates a response which (see derivation in Appendix G), up to corrections of order $1 / \sqrt{K} \tau_{S}$, is given by Eq. (21) (dashed 


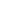

line in Fig. 6B). Similarly to what happens in networks 805 with instantaneous synapses and $a \sim K^{-\alpha}$, this finite 806 response emerges because recurrent interactions set $\mu{ }_{807}$ very close to threshold, at a distance $\theta-\mu \sim 1 / \sqrt{K}{ }_{808}$ that matches the size of the membrane potential fluc- 809 tuations (Eq. 25), $\sigma \sqrt{\tau / \tau_{S}} \sim 1 / \sqrt{K}$ ). In addition, 810 the network becomes much more sensitive to connec- 811 tion heterogeneity, with $C V_{K}^{*} \sim 1 / \sqrt{K}$. However, here ${ }_{812}$ the dynamics of the single neuron membrane potential ${ }_{813}$ is correlated over a timescale $\tau_{S}$ (Fig. 6 $6 \mathrm{E}$ ) and firing is ${ }_{814}$ bursty, with periods of regular spiking randomly inter- 815 spersed in time. Moreover, the properties discussed here ${ }_{816}$ are independent of the scaling of $a$ with $K$, since they ${ }_{817}$ always emerge once $\tau_{S} / \tau \gg 1$, a condition that is met ${ }_{818}$ for any scaling once $\nu_{X} \gg 1 / a K \tau_{S}$. The specific scaling 819 relation, on the other hand, is important to determine 820 the input strength at which $\tau_{S} / \tau \sim 1$.

In the previous sections, we have shown that net- 822 works of conductance-based neurons with instantaneous 823 synapses present features similar to those observed in ${ }^{824}$ cortex if synaptic efficacy is of order $a \sim 1 / \log (K)$, while other scalings generate network properties that are at odds with experimental data (see Tab. II for a summary). In this section, we have found that, when the synaptic time constant is considered, these properties are preserved in the model for low inputs. As the input increases, the structure of the network response evolves gradually and, for large inputs $\left(\nu_{X} \gg 1 / a K \tau_{S}\right)$, significant deviations from the case of instantaneous synapses emerge (see Tab. If for a summary). In particular, as the input to the network increases, our analysis shows that: the membrane potential approaches threshold while its fluctuations become smaller and temporally correlated; firing becomes more bursty; the network becomes more sensitive to heterogeneity in the in-degree and, if the heterogeneity is larger than that of random networks, significant fractions of neurons become quiescent or fire regularly. These features of the model provide a list of predictions which could be tested experimentally by measuring the evolution of the membrane potential dynamics of cells in cortex with the intensity of inputs to the network.

\section{DISCUSSION}

In this work, we analyzed networks of strongly coupled 851 conductance-based neurons. The study of this regime is 852 motivated by the experimental observation that in cor- 853 tex $K$ is large, with single neurons receiving inputs from 854 hundreds or thousands of pre-synaptic cells. We showed 855 that the classical balanced state idea [5, 6, which was 856 developed in the context of current-based models and ${ }_{857}$ features synaptic strength of order $1 / \sqrt{K}[7,8$, results 858 in current fluctuations of very small amplitude, which 859 can generate firing in networks only if the mean mem- 860 brane potential is extremely close to threshold. This ${ }_{861}$ seems problematic since intracellular recordings in cor- ${ }_{862}$ tex show large membrane potential fluctuations (see e.g. 47 52 ). To overcome this problem, we introduced a new scaling relation which, in the case of instantaneous synaptic currents, maintains firing by preserving the balance of input drift and diffusion at the single neuron level. Assuming this scaling, the network response automatically shows multiple features that are observed in cortex in vivo: irregular firing, wide distribution of rates, membrane potential with non-negligible distance from threshold and fluctuation size. When finite synaptic time constants are included in the model, we showed that these properties are preserved for low inputs, but are gradually modified as inputs increase: the membrane mean approaches threshold while its fluctuations decrease in size and develop non-negligible temporal correlations. These properties, which are summarized in Tab. I. provide a list of predictions that could be tested experimentally by analyzing the membrane potential dynamics as a function of input strength in cortical neurons.

When synaptic time constants are negligible with respect to the membrane time constant, our theory shows properties that are analogous to those of the classical balanced state model: linear transfer function, CV of order one, and distribution of membrane potentials with finite width. However, these properties emerge from a different underlying dynamics than in the current based model. In current-based models, the mean input current is at distance of order one from threshold in units of input fluctuations. In conductance-based models, this distance increases with coupling strength and firing is generated by large fluctuations at strong coupling. The different operating mechanism manifests itself in two ways: the strength of synapses needed to sustain firing and the robustness to connection heterogeneity, as we discuss in the next paragraphs.

The scaling relation determines how strong synapses should be to allow firing at a given firing rate, for a given a value of $K$. In current-based neurons, irregular firing is produced as long as synaptic strengths are of order $1 / \sqrt{K}$. In conductance-based neurons, stronger synapses are needed, with a scaling which approaches $1 / \log (K)$ for large $K$. We showed that both scaling relations are in agreement with data obtained from culture preparations 62, which are limited to relatively small networks, and argued that differences might be important in vivo, where $K$ should be larger.

In current-based models, the mean input current must be set at an appropriate level to produce irregular firing; this constraint is realized by recurrent dynamics in networks with random connectivity and strong enough inhibition 7 7 9. However, in networks with structural heterogeneity, with connection heterogeneity larger than $1 / \sqrt{K}$, the variability in mean input currents produces significant departures from the asynchronous irregular state, with large fractions of neurons that become silent or fire regularly [57. This problem is relevant in cortical networks [57, where significant heterogeneity of 


\begin{tabular}{|c|c|c|c|c|c|}
\hline Synaptic model & $\begin{array}{c}\text { Ratio of synaptic } \\
\text { and membrane } \\
\text { time constant }\left(\tau_{S} / \tau\right)\end{array}$ & $\begin{array}{l}\text { Synaptic } \\
\text { strength }\end{array}$ & $\begin{array}{c}\text { Membrane potential } \\
\text { statistics }\end{array}$ & $\begin{array}{l}\text { Activity } \\
\text { structure }\end{array}$ & $\begin{array}{c}\text { Heterogeneity } \\
\text { of in-degree } \\
\text { supported }\left(C V_{K}^{*}\right) \\
\end{array}$ \\
\hline $\begin{array}{c}\text { Current-based } \\
\text { (balanced state model) }\end{array}$ & $\begin{array}{c}\text { constant, } \\
\text { independent of } \\
\nu_{X}, a, \text { and } K\end{array}$ & $J \sim \frac{1}{\sqrt{K}}$ & $\begin{array}{c}\theta-\mu \sim \sigma_{V} \sim 1 \\
\tau_{V} \sim \tau_{L}\end{array}$ & $\begin{array}{l}\text { Irregular firing, } \\
\mathrm{CV} \text { of ISI } \sim 1\end{array}$ & $\sim \frac{1}{\sqrt{K}}$ \\
\hline \multirow[t]{3}{*}{ Conductance-based } & \multirow{2}{*}{$\begin{array}{c}\ll 1 \text { for } \nu_{X} \ll \frac{1}{a K \tau_{S}} ; \\
\text { always satisfied } \\
\text { for instantaneous } \\
\text { synapses }\left(\tau_{S}=0\right)\end{array}$} & $a \sim \frac{1}{\log K}$ & $\begin{array}{c}\theta-\mu \sim 1 ; \\
\sigma_{V} \sim \frac{1}{\sqrt{\log K}} ; \\
\tau_{V} \sim \log (K) / K\end{array}$ & $\begin{array}{l}\text { Irregular firing, } \\
\mathrm{CV} \text { of ISI } \sim 1\end{array}$ & $\sim \frac{1}{\log K}$ \\
\hline & & $\begin{array}{c}a \sim K^{-\alpha} \\
\alpha>0\end{array}$ & $\begin{array}{c}\theta-\mu \sim \sigma_{V} \sim K^{\frac{-\alpha}{2}} \\
\tau_{V} \sim K^{\alpha-1}\end{array}$ & $\begin{array}{l}\text { Irregular firing, } \\
\mathrm{CV} \text { of ISI } \sim 1\end{array}$ & $\sim K^{-\alpha}$ \\
\hline & $\gg 1$ for $\nu_{X} \gg \frac{1}{a K \tau_{S}}$ & any scaling & $\begin{array}{c}\theta-\mu \sim \sigma_{V} \sim \frac{1}{\sqrt{K}} \\
\tau_{V} \sim \tau_{S}\end{array}$ & Irregular bursting & $\sim \frac{1}{\sqrt{K}}$ \\
\hline
\end{tabular}

TABLE I. Overview of of networks of current-based and conductance-based neurons. Synaptic strength and time constant strongly affect response properties in networks of conductance based neurons. Properties similar to what is observed in cortex emerge in these networks if $a \sim 1 / \log K$ and input rates are lower than a critical value, which is fixed by synaptic time constant and coupling strength. The model predicts that these properties should gradually mutate as the input to the network increases and, for large inputs, should coincide with those indicated in the last line of the table. In the table, the different quantities related to the membrane potential represent: the mean distance from threshold $(\theta-\mu)$; the size of temporal fluctuations $\left(\sigma_{V}\right)$; the membrane potential correlation time constant $\left(\tau_{V}\right)$.

in-degrees as been reported [51, 58 61, and different 897 mechanisms have been proposed to solve it [57]. Here 898 we showed that networks of conductance-based neurons 899 also generate irregular activity without any need for fi- 900 nite tuning, and furthermore can support irregular ac- 901 tivity with substantial structural heterogeneity, up to 902 order $1 / \log (K)$. Therefore, these networks are more ro- ${ }_{903}$ bust to connection heterogeneity than the current-based 904 model, and do not need the introduction of additional 905 mechanism to sustain the asynchronous irregular state. 906

The strength of coupling in a network, both in the 907 current-based model [71, 72, and in the conductance- 908 based model (e.g. Fig. 3) determines the structure of 909 its response and hence the computations it can imple- 910 ment. Recent theoretical work, analyzing experimental 911 data in the framework of current-based models, has sug- 912 gested that cortex operates in a regime of moderate cou- ${ }_{913}$ pling [44, 45, where response nonlinearities are promi- 914 nent. In conductance-based models, the effective mem- 915 brane time constant can be informative on the strength 916 of coupling in a network, as it decreases with coupling ${ }_{917}$ strength. Results from in vivo recordings in cat pari- ${ }_{918}$ etal cortex [21] showed evidence that single neuron re- ${ }_{919}$ sponse is sped up by network interactions. In particular, 920 measurements are compatible with inhibitory conduc- ${ }_{921}$ tance approximately 3 times larger than leak conduc- ${ }_{922}$ tance and support the idea that cortex operates in a ${ }_{923}$ "high-conductance state" 22] (but see [23] and discus- ${ }_{924}$ sion below). This limited increase in conductance sup- 925 ports the idea of moderate coupling in cortical networks, 926 in agreement with what found in previous work [44, 45]. ${ }_{927}^{926}$

When the synaptic time constant is much larger than ${ }_{928}$ the membrane time constant, we showed that, regard- ${ }_{929}$ less of synaptic strength, the size of membrane poten- 930 tial fluctuations decreases and firing in the network is preserved by a reduction of the distance from threshold of the mean membrane potential. Moreover, the robustness to heterogeneity in connection fluctuations decreases substantially (the maximum supported heterogeneity becomes of order $1 / \sqrt{K}$ ) and the membrane potential dynamics becomes correlated over a time scale fixed by the synaptic time constant. For really strong coupling, the regime of large synaptic time constant is reached for low input rates. In the case of moderate coupling, which is consistent with experimental data on cortical networks 44, 45, the network response at low rates is well approximated by that of networks with instantaneous synapses, and the regime of large synaptic time constant is reached gradually, as the input to the network increases (Fig. 6). This observation provides a list of prediction on how properties of cortical networks should evolve with input strength (summary in Tab. I), that are testable experimentally.

Experimental evidence suggests that the response to multiple inputs in cortex is non-linear (for an overview, see [73). Such nonlinearities, which are thought to be fundamental to perform complex computations, cannot be captured by the classical balanced state model, as it features a linear transfer function [7, 8. Alternative mechanisms have been proposed [71, 73, 74, but their biophysical foundations [71, 73] or their ability to capture experimentally observed nonlinearities 74 are still not fully understood. We have recently shown 72 that, in networks of current-based spiking neurons, nonlinearities compatible with those used in [71, 73] to explain phenomenology of inputs summation in cortex emerge at moderate coupling. Here we have shown that, as in the case of networks of current-base neurons 72 , nonlin- 
ear responses appears in networks of conductance-based ${ }_{957}$ neurons at moderate coupling, both at response onset ${ }_{958}$ and close to single neuron saturation. In addition, we 959 have found that synaptic time constants provide an ad- 960 ditional source of nonlinearity, with nonlinear responses 961 emerging as the network transitions between the two 962 regimes described above. A full classification of the ${ }_{963}$ nonlinearities generated in these networks is outside the ${ }_{964}$ scope of this work, but could be performed generalizing 965 the approach developed in 72 .

Recent whole cell recording have reported that an in- 968 trinsic voltage-gated conductance, whose strength de- 969 creases with membrane potential, contributes to the 970 modulation of neuronal conductance of cells in pri- 971 mary visual cortex of awake macaques and anesthetized mice 23]. For spontaneous activity, this intrinsic conductance is the dominant contribution to the cell con- 972 ductance and drives its (unexpected) decrease with increased depolarization. For activity driven by sen- 973 sory stimuli, on the other hand, modulations coming 974 from synaptic interactions overcome the effect of the in- 975 trinsic conductance and neuronal conductance increases 976 with increased depolarization. Our analysis shows that 977 voltage-dependent currents, such as that produced by 978 the voltage-gated channels [23] or during spike gener- 979 ation 43, affect quantitatively, but not qualitatively, 980 the single neuron response and the scaling relation allowing firing. Therefore, the results we described in this contribution seem to be a general property of networks of strongly coupled integrate-and-fire neurons with conductance-based synapses.

Understanding the dynamical regime of operation of the cortex is an important open question in neuroscience, as it constrains which computations can be performed by a network 71. Most of the theories of neural networks have been derived using rate models or currentbased spiking neurons. Our work provides the first theory of the dynamics of strongly coupled conductancebased neurons, it can be easily related to measurable quantities because of its biological details, and suggests predictions that could be tested experimentally.

\section{ACKNOWLEDGMENTS}

Supported by the NIMH Intramural Research Program and by NIH BRAIN U01 NS108683 (to M.H. and N.B.). We thank Alex Reyes for providing his data, and Vincent Hakim and Magnus Richardson for comments on the manuscript. This work used the computational resources of the NIH HPC Biowulf cluster (http://hpc.nih.gov) and the Duke Compute Cluster (https://rc.duke.edu/dcc).
[1] Rodney J. Douglas and Kevan A. C. Martin. A func- 1011 tional microcircuit for cat visual cortex. Journal of 1012 Physiology, 440:735-769, 1991.

[2] ZF Mainen and TJ Sejnowski. Reliability of spike timing 1014 in neocortical neurons. Science, 268(5216):1503-1506, 1015 1995.

1016

[3] WR Softky and C Koch. The highly irregular firing of 1017 cortical cells is inconsistent with temporal integration of 1018 random epsps. Journal of Neuroscience, 13(1):334-350, 1019 1993.

[4] Albert Compte, Christos Constantinidis, Jesper Tegnér, 1021 Sridhar Raghavachari, Matthew V. Chafee, Patricia S. 1022 Goldman-Rakic, and Xiao-Jing Wang. Temporally ir- 1023 regular mnemonic persistent activity in prefrontal neu- 1024 rons of monkeys during a delayed response task. Journal 1025 of Neurophysiology, 90(5):3441-3454, 2003. $\overline{\text { PMID: }} 1026$ 12773500.

[5] Michael N. Shadlen and William T. Newsome. Noise, 1028 neural codes and cortical organization. Current Opinion ${ }_{1029}$ in Neurobiology, 4(4):569 - 579, 1994.

[6] Michael N. Shadlen and William T. Newsome. The vari- 1031 able discharge of cortical neurons: implications for con- 1032 nectivity, computation, and information coding. Journal 1033 of Neuroscience, 18(10):3870-3896, 1998.

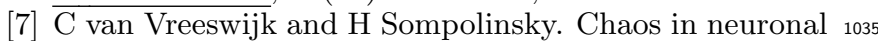
networks with balanced excitatory and inhibitory activ- 1036 ity. Science, 274(5293):1724-6, 1996.

[8] C van Vreeswijk and H Sompolinsky. Chaotic balanced ${ }_{1038}$ state in a model of cortical circuits. Neural computation, 1039 10(6):1321-71, 1998.
[9] D J Amit and N Brunel. Model of global spontaneous activity and local structured activity during delay periods in the cerebral cortex. Cerebral cortex (New York, N.Y. : 1991), 7(3):237-252, 1997.

[10] N Brunel. Dynamics of sparsely connected networls of excitatory and inhibitory neurons. Journal of Computational Neuroscience, 8:183-208, 2000.

[11] Magnus J E Richardson. Effects of synaptic conductance on the voltage distribution and firing rate of spiking neurons. Physical review. E, Statistical, nonlinear, and soft matter physics, 69(5 Pt 1):051918, 2004.

[12] Charles Capaday and Carl van Vreeswijk. Direct control of firing rate gain by dendritic shunting inhibition. Journal of Integrative Neuroscience, 05(02):199222,2006 .

[13] A Destexhe, M Rudolph, J M Fellous, and T J Sejnowski. Fluctuating synaptic conductances recreate in vivo-like activity in neocortical neurons. Neuroscience, 107(1):13-24, 2001.

[14] Michael Shelley, David McLaughlin, Robert Shapley, and Jacob Wielaard. States of high conductance in a large-scale model of the visual cortex. Journal of Computational Neuroscience, 13(2):93-109, Sep 2002.

[15] M Rudolph and A Destexhe. Characterization of subthreshold voltage fluctuations in neuronal membranes. Neural Comput, 15(11):2577-2618, Nov 2003.

[16] Alexander Lerchner, Mandana Ahmadi, and John Hertz. High-conductance states in a mean-field cortical network model. Neurocomputing, 58-60:935 - 940, 2004. Computational Neuroscience: Trends in Research 2004. 
[17] Hamish Meffin, Anthony N. Burkitt, and David B. Gray- 1105 den. An analytical model for the "large, fluctuating 1106 synaptic conductance state" typical of neocortical neu- 1107 rons in vivo. Journal of Computational Neuroscience, 1108 16(2):159-175, 2004.

[18] Michael Rudolph, Joe Guillaume Pelletier, Denis Paré, 1110 and Alain Destexhe. Characterization of synaptic con- 1111 ductances and integrative properties during electrically 1112 induced eeg-activated states in neocortical neurons in 1113 vivo. Journal of Neurophysiology, 94(4):2805-2821, 1114 2005. PMID: 16014785.

[19] A. Kumar, S. Schrader, A. Aertsen, and S. Rotter. 1116 The high-conductance state of cortical networks. Neural 1117 Computation, 20(1):1-43, 2008.

[20] D Pare, E Shink, H Gaudreau, A Destexhe, and E J 1119 Lang. Impact of spontaneous synaptic activity on the 1120 resting properties of cat neocortical pyramidal neurons 1121 in vivo. J Neurophysiol, 79(3):1450-1460, Mar $1998 . \quad 1122$

[21] Alain Destexhe and Denis Paré. Impact of network ac- 1123 tivity on the integrative properties of neocortical pyra- 1124 midal neurons in vivo. Journal of Neurophysiology, 1125 81(4):1531-1547, 1999. PMID: 10200189.

[22] Alain Destexhe, Michael Rudolph, and Denis Paré. The ${ }_{1127}$ high-conductance state of neocortical neurons in vivo. 1128 Nature Reviews Neuroscience, 4(9):739-751, sep 2003. 1129

[23] Baowang Li, Brandy N. Routh, Daniel Johnston, Eyal 1130 Seidemann, and Nicholas J. Priebe. Voltage-gated in- 1131 trinsic conductances shape the input-output relationship 1132 of cortical neurons in behaving primate v1. Neuron, ${ }_{1133}$ 107(1):185 - 196.e4, 2020.

[24] Tim P. Vogels and L. F. Abbott. Signal propagation and 1135 logic gating in networks of integrate-and-fire neurons. 1136 Journal of Neuroscience, 25(46):10786-10795, 2005.

[25] Mark H. Histed. Feedforward inhibition allows in- 1138 put summation to vary in recurrent cortical networks. 1139 eNeuro, 2018.

[26] Stefano Cavallari, Stefano Panzeri, and Alberto Maz- ${ }_{1141}$ zoni. Comparison of the dynamics of neural inter- 1142 actions between current-based and conductance-based 1143 integrate-and-fire recurrent networks. Frontiers in 1144 Neural Circuits, 8:12, 2014.

[27] Nicolas Brunel and Xiao-Jing Wang. Effects of neuro- 1146 modulation in a cortical network model of object work- 1147 ing memory dominated by recurrent inhibition. Journal 1148 of Computational Neuroscience, 11(1):63-85, 2001.

[28] Yann Zerlaut, Sandrine Chemla, Frederic Chavane, and 1150 Alain Destexhe. Modeling mesoscopic cortical dynam- 1151 ics using a mean-field model of conductance-based net- 1152 works of adaptive exponential integrate-and-fire neu- 1153 rons. Journal of Computational Neuroscience, 44(1):45- 1154 $61,2018$.

[29] Christopher Ebsch and Robert Rosenbaum. Imbalanced 1156 amplification: A mechanism of amplification and sup- 1157 pression from local imbalance of excitation and inhibi- 1158 tion in cortical circuits. PLOS Computational Biology, 1159 14(3):1-28, 032018.

[30] Cristiano Capone, Matteo di Volo, Alberto Romagnoni, ${ }_{1161}$ Maurizio Mattia, and Alain Destexhe. State-dependent 1162 mean-field formalism to model different activity states in 1163 conductance-based networks of spiking neurons. Phys. ${ }_{1164}$ Rev. E, 100:062413, Dec 2019.

[31] Matteo di Volo, Alberto Romagnoni, Cristiano Capone, 1166 and Alain Destexhe. Biologically realistic mean-field 1167 models of conductance-based networks of spiking neu- 1168 rons with adaptation. Neural Computation, 31(4):653680, 2019. PMID: 30764741.

[32] Randy M. Bruno and Bert Sakmann. Cortex is driven by weak but synchronously active thalamocortical synapses. Science, 312(5780):1622-1627, 2006.

[33] Henry Markram, Joachim Lubke, Michael Frotscher, and Bert Sakmann. Regulation of synaptic efficacy by coincidence of postsynaptic APs and EPSPs. Science, 275(5297):213-215, jan 1997.

[34] Per Jesper Sjöström, Gina G Turrigiano, and Sacha B Nelson. Rate, timing, and cooperativity jointly determine cortical synaptic plasticity. Neuron, 32(6):11491164, dec 2001.

[35] Carl Holmgren, Tibor Harkany, Björn Svennenfors, and Yuri Zilberter. Pyramidal cell communication within local networks in layer $2 / 3$ of rat neocortex. Journal of Physiology, 551(1):139-153, 2003.

[36] Sandrine Lefort, Christian Tomm, J. C. Floyd Sarria, and Carl C.H. Petersen. The excitatory neuronal network of the $\mathrm{C} 2$ barrel column in mouse primary somatosensory cortex. Neuron, 61(2):301-316, 2009.

[37] Rodrigo Perin, Thomas K Berger, and Henry Markram. A synaptic organizing principle for cortical neuronal groups. Proceedings of the National Academy of Sciences, 108(13):5419-5424, 2011.

[38] Xiaolong Jiang, Shan Shen, Cathryn R Cadwell, Philipp Berens, Fabian Sinz, Alexander S Ecker, Saumil Patel, and Andreas S Tolias. Principles of connectivity among morphologically defined cell types in adult neocortex. Science, 350(6264):aac9462-aac9462, 2015.

[39] Magnus J. E. Richardson and Wulfram Gerstner. Synaptic shot noise and conductance fluctuations affect the membrane voltage with equal significance. Neural Computation, 17(4):923-947, 2005.

[40] Arnold J. F. Siegert. On the first passage time probability problem. Phys. Rev., 81:617-623, Feb 1951.

[41] Luigi M. Ricciardi. Diffusion processes and related topics in biology. Springer-Verlag Berlin ; New York, 1977.

[42] Crispin Gardiner. Stochastic Methods. Springer, 4th ed. 2009 edition, January 2009.

[43] Nicolas Fourcaud-Trocmé, David Hansel, Carl van Vreeswijk, and Nicolas Brunel. How spike generation mechanisms determine the neuronal response to fluctuating inputs. Journal of Neuroscience, 23(37):1162811640, 2003.

[44] Alessandro Sanzeni, Bradley Akitake, Hannah C Goldbach, Caitlin E Leedy, Nicolas Brunel, and Mark H Histed. Inhibition stabilization is a widespread property of cortical networks. eLife, 9:e54875, 062020.

[45] Yashar Ahmadian and Kenneth D. Miller. What is the dynamical regime of cerebral cortex? arXiv, page 1908.10101, 2019.

[46] Nicolas Brunel and Vincent Hakim. Fast global oscillations in networks of integrate-and-fire neurons with low firing rates. Neural Computation, 11(7):1621-1671, 1999.

[47] Jeffrey Anderson, Ilan Lampl, Iva Reichova, Matteo Carandini, and David Ferster. Stimulus dependence of two-state fluctuations of membrane potential in cat visual cortex. Nature Neuroscience, 3(6):617-621, 2000.

[48] Sylvain Crochet and Carl C H Petersen. Correlating whisker behavior with membrane potential in barrel cortex of awake mice. Nature Neuroscience, 9(5):608-610, 
2006.

[49] James F. A. Poulet and Carl C. H. Petersen. Internal ${ }_{1233}$ brain state regulates membrane potential synchrony in 1234 barrel cortex of behaving mice. Nature, 454(7206):881- 1235 $885,2008$.

[50] Andrew Y. Tan, Yuzhi Chen, Benjamin Scholl, Eyal Sei- 1237 demann, and Nicholas J. Priebe. Sensory stimulation 1238 shifts visual cortex from synchronous to asynchronous 1239 states. Nature, 509:226 EP -, 032014.

[51] Michael Okun, Nicholas Steinmetz, Lee Cossell, M Flo- 1241 rencia Iacaruso, Ho Ko, Peter Bartho, Tirin Moore, 1242 Sonja B Hofer, Thomas D Mrsic-Flogel, Matteo Caran- 1243 dini, and Kenneth D Harris. Diverse coupling of 1244 neurons to populations in sensory cortex. Nature, 1245 521(7553):511-515, May 2015.

[52] Jianing Yu, Diego A Gutnisky, S Andrew Hires, and 1247 Karel Svoboda. Layer 4 fast-spiking interneurons filter 1248 thalamocortical signals during active somatosensation. 1249 Nature Neuroscience, 19(12):1647-1657, 2016.

1250

[53] Tomáš Hromádka, Michael R DeWeese, and Anthony M 1251 Zador. Sparse representation of sounds in the unanes- 1252 thetized auditory cortex. PLOS Biology, 6(1):1-14, 011253 2008.

[54] Daniel H. O'Connor, Simon P. Peron, Daniel Huber, and 1255 Karel Svoboda. Neural activity in barrel cortex under- 1256 lying vibrissa-based object localization in mice. Neuron, 1257 67(6):1048 - 1061, 2010.

[55] György Buzsáki and Kenji Mizuseki. The log-dynamic 1259 brain: how skewed distributions affect network opera- 1260 tions. Nature reviews. Neuroscience, 15(4):264-278, 041261 2014.

[56] Alex Roxin, Nicolas Brunel, David Hansel, Gianluigi ${ }_{1263}$ Mongillo, and Carl van Vreeswijk. On the distribution 1264 of firing rates in networks of cortical neurons. Journal 1265 of Neuroscience, 31(45):16217-16226, $2011 . \quad 1266$

[57] Itamar D. Landau, Robert Egger, Vincent J. Dercksen, 1267 Marcel Oberlaender, and Haim Sompolinsky. The im- ${ }_{1268}$ pact of structural heterogeneity on excitation-inhibition 1269 balance in cortical networks. Neuron, 92(5):1106-1121, 1270 2018/03/09 2016.

[58] Nuno Maçarico da Costa and Kevan A. C. Martin. How 1272 thalamus connects to spiny stellate cells in the cat visual 1273 cortex. Journal of Neuroscience, 31(8):2925-2937, 2011. 1274

[59] Takahiro Furuta, Martin Deschênes, and Takeshi 1275 Kaneko. Anisotropic distribution of thalamocortical 1276 boutons in barrels. The Journal of Neuroscience, 1277 31(17):6432, 042011.

1278

[60] Mingshan Xue, Bassam V Atallah, and Massimo 1279 Scanziani. Equalizing excitation-inhibition ratios across 1280 visual cortical neurons. Nature, 511(7511):596-600, Jul 1281 2014.

1282

[61] Carl E Schoonover, Juan-Carlos Tapia, Verena C ${ }_{1283}$ Schilling, Verena Wimmer, Richard Blazeski, Wanying 1284 Zhang, Carol A Mason, and Randy M Bruno. Compara- 1285 tive strength and dendritic organization of thalamocor- 1286 tical and corticocortical synapses onto excitatory layer 1287 4 neurons. J Neurosci, 34(20):6746-6758, May 2014. ${ }_{1288}$

[62] Jeremie Barral and A Reyes. Synaptic scaling rule pre- 1289 serves excitatory-inhibitory balance and salient neuronal 1290 network dynamics. Nature Neuroscience, 19(12):1690- 1291 1696, October 2016.

[63] Alain Destexhe, Zachary Mainen, and Terrence Sejnowski. Kinetic Models of Synaptic Transmission, vol- ume 2. 011998.

[64] D A McCormick, B W Connors, J W Lighthall, and D A Prince. Comparative electrophysiology of pyramidal and sparsely spiny stellate neurons of the neocortex. J Neurophysiol, 54(4):782-806, Oct 1985.

[65] Hidehiko K. Inagaki, Lorenzo Fontolan, Sandro Romani, and Karel Svoboda. Discrete attractor dynamics underlies persistent activity in the frontal cortex. Nature, 566(7743):212-217, 2019.

[66] Marcel Stimberg, Romain Brette, and Dan Fm Goodman. Brian 2, an intuitive and efficient neural simulator. eLife, 8:e47314, aug 2019.

[67] Nicolas Brunel and Simone Sergi. Firing frequency of leaky intergrate-and-fire neurons with synaptic current dynamics. Journal of Theoretical Biology, 195(1):8795, 1998.

[68] P. I. M. Johannesma. Diffusion models for the stochastic activity of neurons, pages 116-144. Springer Berlin Heidelberg, Berlin, Heidelberg, 1968.

[69] Rubén Moreno-Bote and Néstor Parga. Role of synaptic filtering on the firing response of simple model neurons. Physical Review Letters, 92(2):4, 2004.

[70] Laurent Badel. Firing statistics and correlations in spiking neurons: A level-crossing approach. Physical Review E, 84(4):041919, oct 2011.

[71] Yashar Ahmadian, Daniel B Rubin, and Kenneth D Miller. Analysis of the stabilized supralinear network. Neural Computation, 25(8):1994-2037, 2013.

[72] Alessandro Sanzeni, Mark H. Histed, and Nicolas Brunel. Response nonlinearities in networks of spiking neurons. PLOS Computational Biology, 16(9):1-27, 09 2020.

[73] Daniel B. Rubin, Stephen D. VanHooser, and Kenneth D. Miller. The stabilized supralinear network: A unifying circuit motif underlying multi-input integration in sensory cortex. Neuron, 85(2):402-417, 2015.

[74] Cody Baker, Vicky Zhu, and Robert Rosenbaum. Nonlinear stimulus representations in neural circuits with approximate excitatory-inhibitory balance. bioRxiv, 2019.

[75] Magnus J E Richardson. Firing-rate response of linear and nonlinear integrate-and-fire neurons to modulated current-based and conductance-based synaptic drive. Physical Review E - Statistical, Nonlinear, and Soft Matter Physics, 76(2):1-15, 2007.

[76] Francesca Barbieri and Nicolas Brunel. Irregular persistent activity induced by synaptic excitatory feedback. Frontiers in Computational Neuroscience, 1:5, 2007.

[77] Bard Ermentrout and David Terman. The Mathematical Foundations of Neuroscience, volume $\overline{35 .}$ 072010.

[78] Daniel J. Amit and Nicolas Brunel. Dynamics of a recurrent network of spiking neurons before and following learning. Network, 8:373-404, 1997.

[79] Carl van Vreeswijk and Farzad Farkhooi. Fredholm theory for the mean first-passage time of integrate-and-fire oscillators with colored noise input. Physical Review E, 100(6), Dec 2019.

[80] S. O. Rice. Mathematical analysis of random noise. Bell System Technical Journal, 23(3):282-332, 1944. 


\section{Appendix A: Calculations in the multiplicative noise case}

In the main text, we analyze the distribution of membrane potential, firing rate and CV using the effective time constant approximation, which neglects the dependence of the noise amplitude on the membrane potential. This approximation is motivated by the fact that corrections to this approximation are of the same order of shot noise corrections to the diffusion approximation used to describe synaptic inputs [75]. In this section, we derive results without resorting to the effective time constant approximation (i.e. keeping the voltage dependence of the noise term), and show that the results derived in the main text remain valid, even though it complicates the calculations. The inclusion of shot noise corrections is outside the scope of this contribution.

\section{Equations for arbitrary drift and diffusion terms}

In this section, we compute the probability distribution of the membrane potential, the firing rate, and the CV of ISI of a neuron whose membrane potential follows the equation

$$
\frac{d V}{d t}=A(V)+B(V) \zeta
$$

Eq. (4) of the main text is a special form of Eq. A1 with

$$
A(V)=\frac{\mu-V}{\tau}, \quad B(V)=\frac{\sigma(V)}{\sqrt{\tau}} .
$$

The Fokker-Plank equation associated to Eq. A1, in the Stratonovich regularization scheme, is given by

$$
\frac{d P}{d t}=-\frac{\partial J}{\partial V}
$$

where $P$ is the probability of finding a neuron with membrane potential $V$ and $J$ is the corresponding probability current given by

$$
J=\left(A+\frac{1}{2} B \frac{\partial B}{\partial V}\right) P-\frac{1}{2} \frac{\partial B^{2} P}{\partial V} .
$$

We are interested in the stationary behavior of the system in which $P$ does not depend on time and the current $J$ is piecewise constant. In particular, for $V$ between the activation threshold $\theta$ and the resting potential $V_{r}, J$ is equal to the neuron firing rate $\nu$ and the normalization condition implies

$$
\int_{V_{r}}^{\theta} P(V) d V+\nu \tau_{r p}=1
$$

where $\tau_{r p}$ is the refractory period.

To derive the probability distribution of the neuron potential, we introduce in Eq. A3 the integrating factor

$$
W(V)=\exp \left[-2 \int^{V} d u \frac{A(u)+\frac{1}{2} B(u) \frac{\partial B(u)}{\partial u}}{B(u)^{2}}\right]
$$

and obtain

$$
-2 \nu W(V) \theta\left(V-V_{r}\right)=\frac{\partial}{\partial V}\left[W(V) B(V)^{2} P(V)\right] .
$$

Using the boundary condition $P(\theta)=0$, we find

$$
P(V)=\frac{2 \nu}{W(V) B(V)^{2}} \int_{V}^{\theta} d u W(u) \theta\left(u-V_{r}\right)
$$

and

$$
\frac{1}{\nu}=\tau_{r p}+2 \int_{-\infty}^{\theta} d x \frac{1}{W(x) B(x)^{2}} \int_{x}^{\theta} d u W(u) \theta\left(u-V_{r}\right)
$$


Integrating by parts, we obtain

$$
\frac{1}{\nu}=\tau_{r p}+2 \int_{V_{r}}^{\theta} d v W(v) \int_{-\infty}^{v} d x \frac{1}{W(x) B(x)^{2}}
$$

This solution has been obtained in general form in [40] and for the specific form of Eq. A2] in [11].

We now compute the coefficient of variation of the interspike interval. The moments $T_{k}$ of the interspike intervals of the stochastic process defined by Eq. A1] are given by (see [42])

$$
\frac{B(x)^{2}}{2} \frac{d^{2} T_{k}(x)}{d x^{2}}+\left(A(x)+\frac{1}{2} B(x) \frac{\partial B(x)}{\partial x}\right) \frac{d T_{k}(x)}{d x}=-k T_{k-1}(x)
$$

with boundary conditions

$$
T_{k}(\theta)=0, \quad \frac{d T_{k}(b)}{d x}=0,
$$

i.e. $\theta$ is an absorbing boundary and $b$ is a reflective boundary (we will then consider the limit $b \rightarrow-\infty$ ). The general solution of an equation of the form

$$
\frac{d^{2} f(x)}{d x^{2}}+P(x) \frac{d f(x)}{d x}=Q(x)
$$

is

$$
f(x)=\int_{\theta}^{x} d t \int_{-\infty}^{t} d z Q(z) \exp \left(\int_{t}^{z} d w P(w)\right) .
$$

For $T_{1}(x)$ we have

$$
P(x)=\frac{2 A(x)+B(x) \frac{\partial B(x)}{\partial x}}{B(x)^{2}}, \quad Q(x)=-\frac{2}{B(x)^{2}}
$$

For $T_{2}(x)$ we look for a solution of the form

$$
T_{2}(x)=T_{1}(x)^{2}+R(x)
$$

and find that $R$ obeys to an equation of the form of Eq. A6 with

$$
P(x)=\frac{2 A(x)+B(x) \frac{\partial B(x)}{\partial x}}{B(x)^{2}}, \quad Q(x)=-2\left(\frac{d T_{1}(x)}{d x}\right)^{2}
$$

Combining the previous results, the CV of ISI is obtained as

$$
C V^{2}=\frac{R(x)}{T_{1}(x)^{2}} ;
$$

the explicit expression of the $C V$ is given in the following section.

Eqs. (17), 10) and 15) of the main text have been obtained from Eqs. A4, (A5) and (A7) using Eq. A2.

\section{Equations for conductance-based LIF neurons}

Starting from Eqs. 45 of the main text, we write the different terms as

$$
\begin{array}{r}
\tau^{-1}=\tau_{L}{ }^{-1}+a K \omega^{-1}, \quad \mu=\tau\left\{E_{L} / \tau_{L}+a K\left[r_{E} E_{E}+r_{I} g \gamma E_{I}\right]\right\}, \\
\sigma^{2}=a^{2} K \frac{\tau}{\chi}\left[\left(V-\mathcal{E}_{\mathcal{S}}\right)^{2}+\mathcal{E}_{\mathcal{D}}{ }^{2}\right],
\end{array}
$$

where, to shorten the expressions, we have introduced two auxiliary variables with time dimension

$$
\omega^{-1}=r_{E}+r_{I} g \gamma, \quad \chi^{-1}=r_{E}+r_{I} g^{2} \gamma,
$$



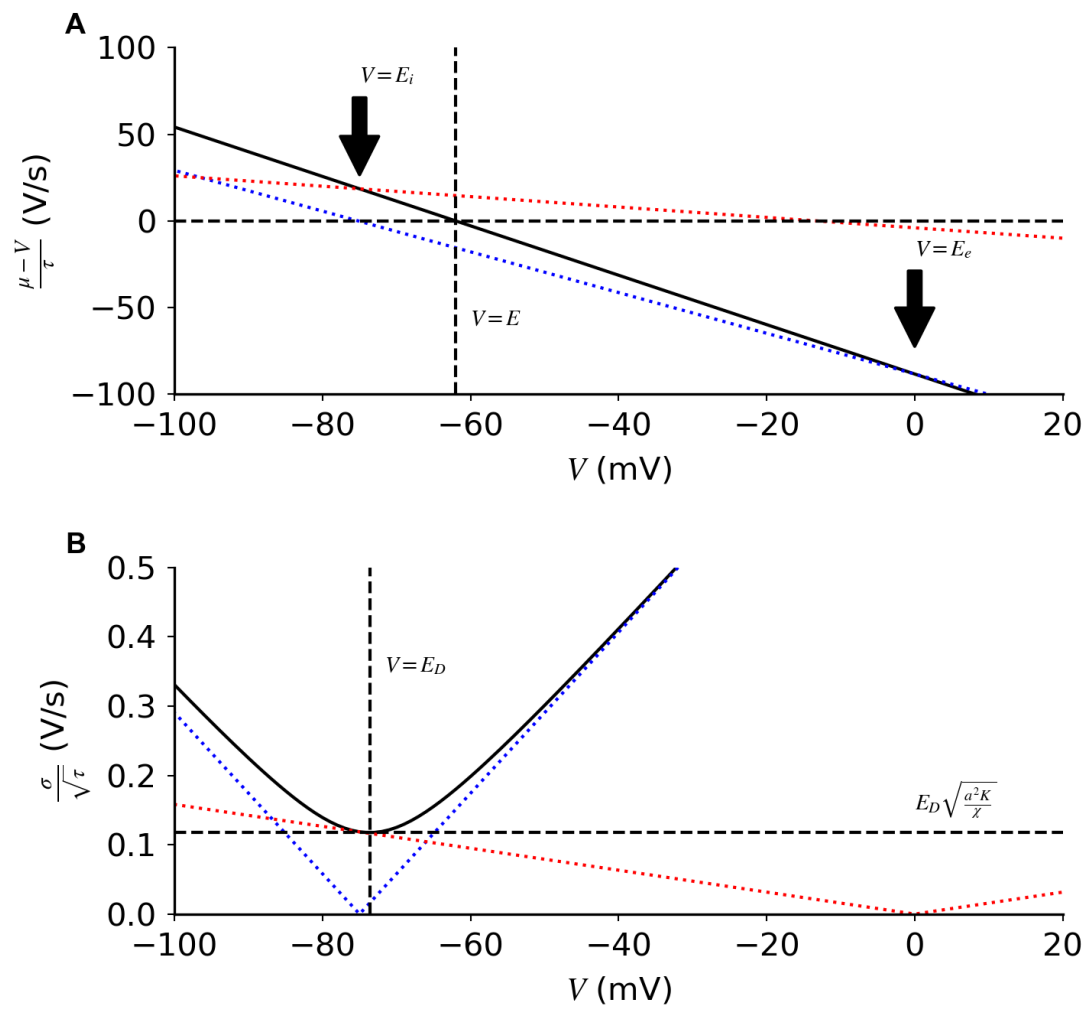

FIG. 7. Drift and diffusion terms of Eq. (4) as a function of voltage. (A) Input drift as a function of membrane potential $V$ produced with both inhibitory and excitatory inputs (black line), excitatory inputs only (red dotted line), or inhibitory inputs only (blue dotted line). The drift term decreases monotonically with $V$ and it is zero at $V=\mu$, which is a stable fixed point of the deterministic dynamics. (B) The noise variance is quadratic in $V$. Its minimum at $V=\mathcal{E}_{\mathcal{S}}$ is equal to $\mathcal{E}_{\mathcal{D}} \sqrt{a^{2} K / \chi}$. Note that the minimum amplitudes of drift and variance are obtained at different values of $V$.

as well as two variables with voltage dimensions,

$$
\mathcal{E}_{\mathcal{S}}=\chi\left(r_{E} E_{E}+r_{I} g^{2} \gamma E_{I}\right), \quad \mathcal{E}_{\mathcal{D}}=\chi \sqrt{r_{E} r_{I} g^{2} \gamma}\left(E_{E}-E_{I}\right) .
$$

The terms $-(V-\mu) / \tau$ and $\sigma(V) \zeta / \sqrt{\tau}$ of Eq. (4) represent the input drift and noise to the membrane dynamics respectively. The voltage dependence of these terms is sketched in Fig. 7.

In the large $K$ limit, the different terms in Eq. (4) scale as

$$
\tau \sim \frac{\omega}{a K}, \quad \mu \sim \omega\left(r_{E} E_{E}+r_{I} g \gamma E_{I}\right), \quad \sigma \sqrt{\tau} \sim \frac{\omega}{\sqrt{\chi K}} \sqrt{\left(V-\mathcal{E}_{\mathcal{S}}\right)^{2}+\mathcal{E}_{\mathcal{D}}^{2}}
$$

while the values of $\omega, \mu, \mathcal{E}_{\mathcal{S}}$, and $\mathcal{E}_{\mathcal{D}}$ are independent of $K$. It follows that the noise term $\sigma \sqrt{\tau}$ and the time constant $\tau$ in Eq. (4) become small in the strong coupling limit. This result is analogous to what we obtained in the main text with the effective time constant approximation, since this approximation does not change how these terms scale with $a$ and $K$.

We now insert the drift and diffusion terms of the conductance-based LIF neuron in Eqs. (A4), (A5), and (A7), and obtain

$$
P(V)=\frac{2 \nu \chi \mathcal{E}_{\mathcal{D}} e^{\frac{-\mathcal{F}(V)}{a}}}{a^{2} K\left[\left(V-\mathcal{E}_{\mathcal{S}}\right)^{2}+\mathcal{E}_{\mathcal{D}}{ }^{2}\right]} \int_{u(V)}^{v_{\max }} d x \theta\left(x-u\left(V_{r}\right)\right) e^{\frac{\mathcal{F}(x)}{a}}
$$

$$
\frac{1}{\nu}=\tau_{r p}+\frac{2 \chi}{a^{2} K} \int_{v_{\min }}^{v_{\max }} d v \int_{-\infty}^{v} d x \frac{1}{x^{2}+1} \exp \left[\frac{\mathcal{F}(v)-\mathcal{F}(x)}{a}\right]
$$


and

$$
C V^{2}=\frac{8 \chi^{2} \nu^{2}}{a^{4} K^{2}} \int_{v_{\min }}^{v_{\max }} d v \int_{-\infty}^{v} d z \exp \left[\frac{\mathcal{F}(v)-\mathcal{F}(z)}{a}\right]\left\{\int_{-\infty}^{z} d w \frac{1}{w^{2}+1} \exp \left[\frac{\mathcal{F}(z)-\mathcal{F}(w)}{a}\right]\right\}^{2}
$$

where

$$
\begin{array}{r}
\mathcal{F}(x)=\frac{2 \chi}{a K \tau}\left[\frac{1}{2}\left(1-\frac{a^{2} K \tau}{2 \chi}\right) \log \left(x^{2}+1\right)-\alpha \arctan (x)\right], \quad u(V)=\frac{V-\mathcal{E}_{\mathcal{S}}}{\mathcal{E}_{\mathcal{D}}}, \\
v_{\min }=u\left(V_{r}\right), \quad v_{\max }=u(\theta), \quad \alpha=u(\mu) .
\end{array}
$$

Eqs. A12 and (A13) are analogous to those derived in [11. To simplify the following analysis, we will neglect the contribution of the term $a^{2} K \tau / 2 \chi$, which derives from the regularization scheme. This assumption is justified by the fact that, for large $K, \tau \sim 1 / a K$ and the factor $a^{2} K \tau / 2 \chi$ is of order $a \ll 1$.

\section{Appendix B: Calculations in the strong coupling regime - Single neurons}

In the main text, we derived a simplified expression for the single neuron response neglecting the dependency of noise on membrane potential. In this section we generalize this result to the case in which the full noise expression is considered. We compute simplified expressions of the single neuron transfer function and CV, both in the subthreshold regime $\mu<\theta$, and the supra-threshold regime $\mu>\theta$. These expressions are validated numerically in Fig. 8 and used in the last part of this section to define a scaling relation between $a$ and $K$ which preserves single neuron firing in the strong coupling limit.
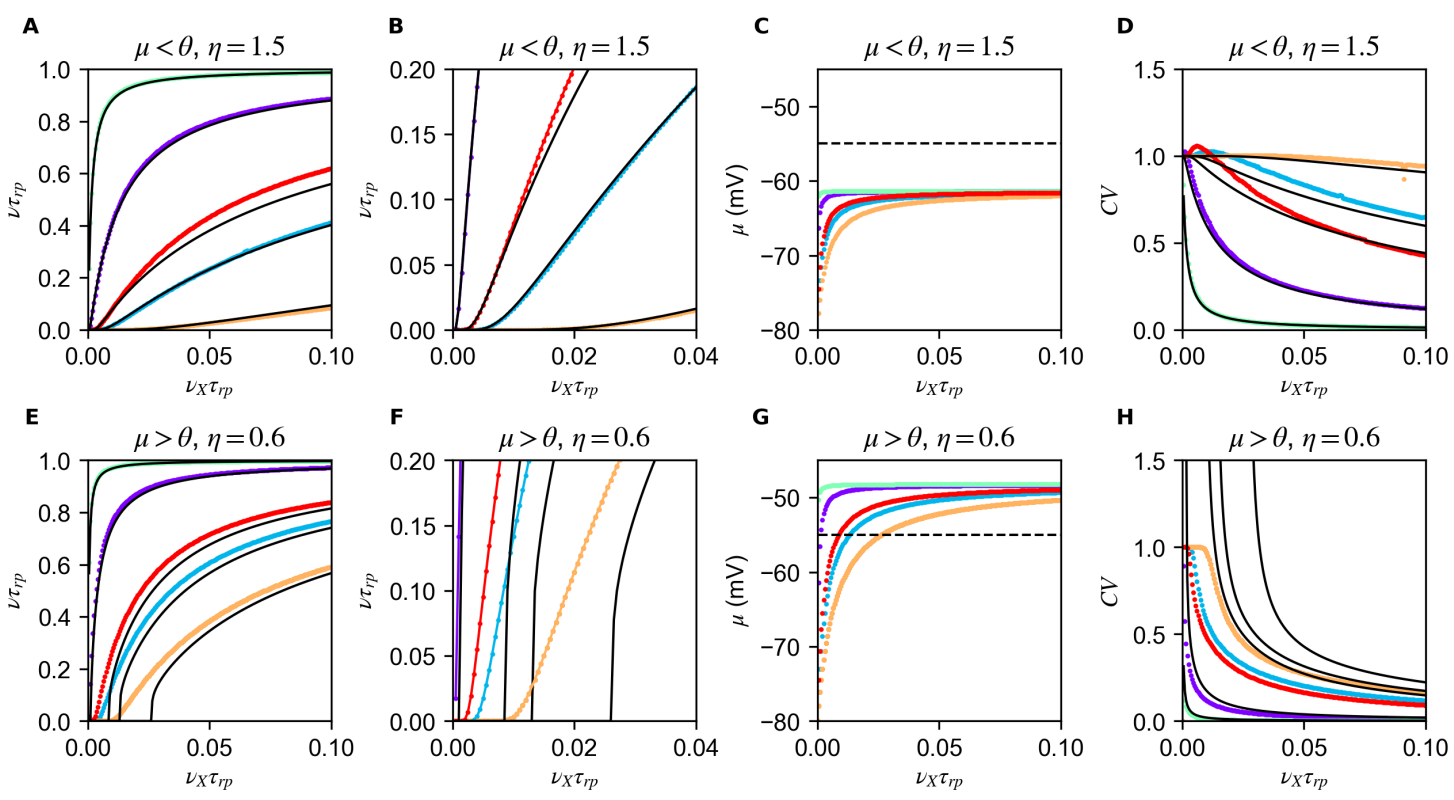

\begin{tabular}{|c|c|c|c|c|}
\hline & $a=1.0 \mathrm{e}-02, K=1.0 \mathrm{e}+04$ & . & $a=1.0 \mathrm{e}-02, K=1.0 \mathrm{e}+05$ & - $a=1.5 \mathrm{e}-02, K=1.0 \mathrm{e}+03$ \\
\hline - & $a=1.0 \mathrm{e}-02, K=1.0 \mathrm{e}+03$ & - & $a=5.0 \mathrm{e}-03, K=1.0 \mathrm{e}+03$ & \\
\hline
\end{tabular}
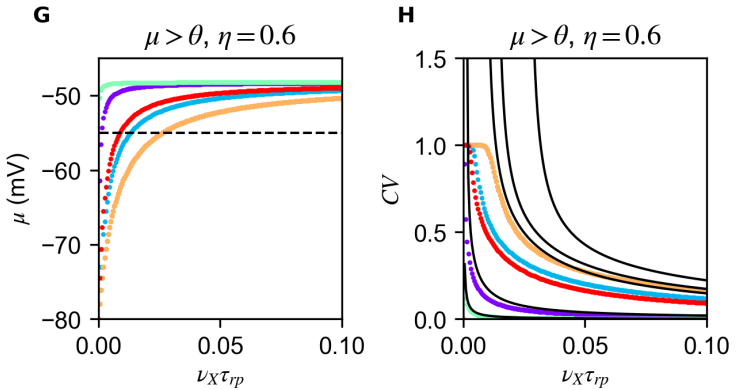

FIG. 8. Response of single conductance-based neuron to noisy inputs. Estimates of firing rate $(\mathbf{A}, \mathbf{B}, \mathbf{E}, \mathbf{F}), \mu(\mathbf{C}$, $\mathbf{G})$ and $C V(\mathbf{D}, \mathbf{H})$ obtained with numerical integration of Eqs A13, (13) and A14 for different values of $a$ and $K$ (colored dots). For the two regimes $\mu<\theta$ (first row) and $\mu>\theta$ (second row), the transfer function saturates as $K$ increases. Note the same change in $a$ has a more drastic effect if $\mu<\theta$, this is due to the exponential dependence that appears in Eq. B6. The approximated expressions (continuous lines) capture the properties of transfer function (A Eq. (B6) and E, Eq. (B4) and $C V$ (C, Eq (B17) and G, Eq (B9)). For small inputs $(\mathbf{F})$, Eq. (B4) fails to describe the transfer function for some values of $K$ because the corresponding $\mu$ is below threshold. Simulations parameter are: $g=12 ; \gamma=1 / 4 ; \eta=1.5$ (top) or 0.6 (bottom). 


\section{Single neuron transfer function at strong coupling}

The starting point of our analysis is the observation that the integrand in Eq. A13 depends exponentially on $1 / a \gg 1$. This suggests to perform the integration with a perturbative expansion of the exponent. We will show below that, since the exponent has a stationary point at $x=v=\alpha$ (see Fig. 9), the integration gives two qualitatively different results if $\alpha$ is larger or smaller than the upper bound of the integral $v_{\max }$. Moreover, since the condition $\alpha \lessgtr v_{\max }$ corresponds to $\theta \lessgtr \mu$, the two behaviors correspond to supra/sub-threshold regimes, respectively.
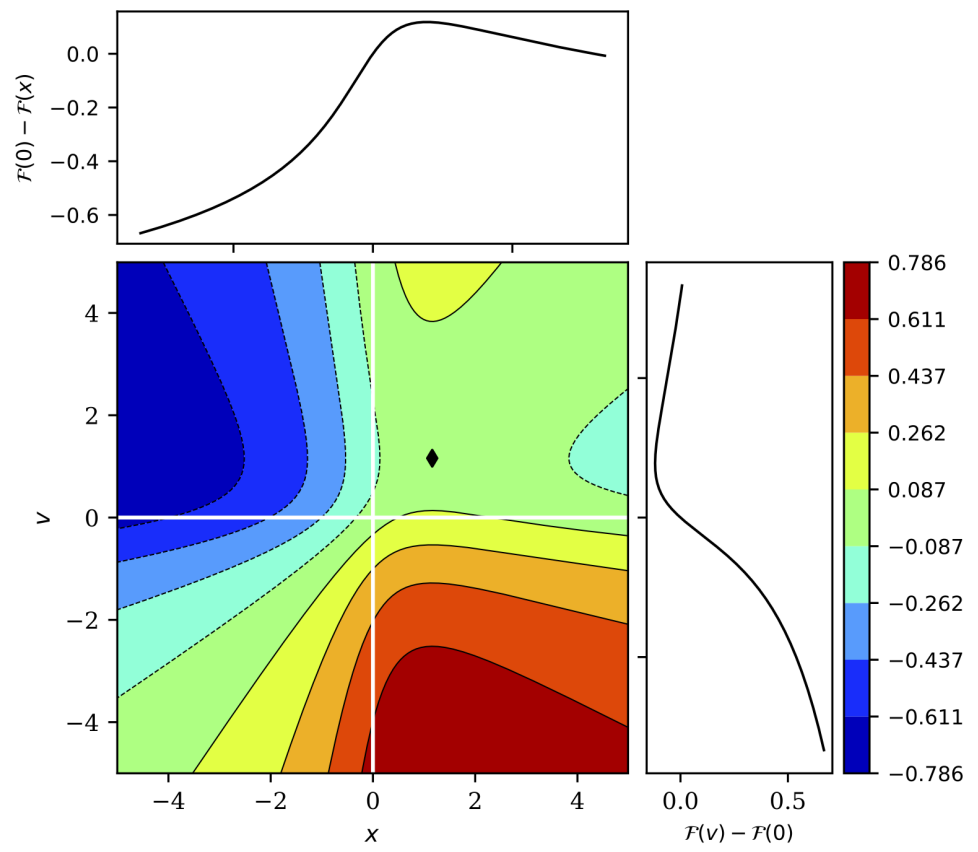

FIG. 9. Graphical representation of the exponent in Eq. A13 The function $\mathcal{F}(v)-\mathcal{F}(x)$ is stationary at $x=v=\alpha$, this point is a maximum for $x$ and a minimum for $v$. Parameters are as in Fig. 7 . In this figure, $\alpha=1.2$ (black diamond).

Supra-threshold regime $v_{\max }<\alpha(\theta<\mu)$

The exponent in Eq. A13 is negative for every value of $x$, except for $x=v$ in which it is zero. The integral in $x$ can be written has

$$
I=\int_{-\infty}^{v} d x g(x) e^{\frac{f_{v}(x)}{a}}=\int_{-\infty}^{v} d x g(x) e^{\frac{1}{a}\left(f_{v}^{\prime}(v)(x-v)+f_{v}^{\prime \prime}(v) / 2(x-v)^{2}+\ldots\right)}
$$

With a change of variable $z=(x-v) / a$ we obtain

$$
I=a \int_{-\infty}^{0} d z g(v+a z) e^{f_{v}^{\prime}(v) z+a f_{v}^{\prime \prime}(v) \frac{z^{2}}{2}+\ldots}
$$

Neglecting all the terms of order $a$ we get

$$
I=a \frac{g(v)}{f_{v}^{\prime}(v)} .
$$

Performing the integration in $v$ we obtain

$$
\frac{1}{\nu}=\tau_{r p}+\tau \log \left(\frac{\mu-V_{r}}{\mu-\theta}\right) .
$$

Eq. (B4) is the transfer function of a deterministic conductance-based neuron with the addition of the refractory period. This is not surprising since the noise term becomes negligible compared to mean inputs in the small $a$ limit. 
In Fig. $8 \mathrm{~B}$ we show that Eq. (B4) gives a good description of the transfer function predicted by the mean field theory in the supra-threshold regime.

Sub-threshold regime $v_{\max }>\alpha(\theta>\mu)$

First we consider $\alpha<v_{\min }\left(\mu<V_{r}\right)$. For every value of $v$, the integral in $x$ in Eq (A13) has a maximum in the integration interval, hence it can be performed through saddle-point method and gives

$$
\frac{1}{\nu}-\tau_{r p}=\sqrt{\frac{4 \pi \chi \tau}{a^{2} K\left(\alpha^{2}+1\right)}} \int_{v_{\min }}^{v_{\max }} d v \exp \left[\frac{\mathcal{F}(v)-\mathcal{F}(\alpha)}{a}\right] .
$$

In the last equation, the exponent in the integrand has a minimum for $v=\alpha$ and is maximum at $v=v_{\max }$; we expand the exponent around $v=v_{\max }$ and, keeping term up to the first order, obtain

$$
\frac{1}{\nu}-\tau_{r p}=\tau \sqrt{\frac{\pi a^{2} K \tau}{\chi\left(\alpha^{2}+1\right)}} \frac{v_{\max }^{2}+1}{\left|v_{\max }-\alpha\right|} \exp \left[\frac{\mathcal{F}\left(v_{\max }\right)-\mathcal{F}(\alpha)}{a}\right] .
$$

In the regime $v_{\min }<\alpha<v_{\max }$, the integral in $v$ of Eq. A13) can be divided into three parts

$$
\int_{v_{\min }}^{v_{\max }} d v=\int_{v_{\min }}^{\alpha-\epsilon} d v+\int_{\alpha-\epsilon}^{\alpha+\epsilon} d v+\int_{\alpha+\epsilon}^{v_{\max }} d v
$$

the third integral is analogous to case $\alpha<v_{\min }$, hence it has an exponential dependency on the parameters and dominates the other terms. In Fig. 8A we show that Eq. (B6) gives a good description of the transfer function predicted by the mean field theory for $\mu<\theta$.

\section{Single neuron $C V$ of ISI at strong coupling}

In this section we provide details of the derivation the approximated expressions of the response $C V$. Starting from the mean field result of Eq. A14, we compute integrals using the approach discussed above.

Suprathreshold regime $v_{\max }<\alpha(\theta<\mu)$

The inner integral in Eq. A14 yields in the small $a$ limit

$$
\int_{-\infty}^{z} d w \frac{1}{w^{2}+1} \exp \left[\frac{\mathcal{F}(z)-\mathcal{F}(w)}{a}\right]=\frac{a}{z^{2}+1} \frac{1}{\frac{d \mathcal{F}(z)}{d z}}
$$

from which we obtain

$$
C V^{2}=a \frac{\nu^{2}(a K \tau)^{3}}{a^{2} K^{2} \chi}\left[\log \left(\frac{v_{\min }-\alpha}{v_{\max }-\alpha}\right)+\frac{-3 \alpha^{2}+4 \alpha v_{\max }+1}{2\left(\alpha-v_{\max }\right)^{2}}-\frac{-3 \alpha^{2}+4 \alpha v_{\min }+1}{2\left(\alpha-v_{\min }\right)^{2}}\right]
$$

hence the rescaling needed to preserve the deterministic component $a \sim 1 / K$ produces $C V^{2} \sim a \ll 1$. We validated this result numerically in Figs. $8 \mathrm{H}$ and $10 \mathrm{~F}$.

Subthresold regime $v_{\max }>\alpha(\theta>\mu)$

The integral defining the $C V$, Eq. A14, can be expressed as

$$
\int_{-\infty}^{v} d z \exp \left[\frac{\mathcal{F}(v)-\mathcal{F}(z)}{a}\right] g(z)=\int_{-\infty}^{v^{*}} d z \exp \left[\frac{\mathcal{F}(v)-\mathcal{F}(z)}{a}\right] g(z)+\int_{v^{*}}^{v} d z \exp \left[\frac{\mathcal{F}(v)-\mathcal{F}(z)}{a}\right] g(z)
$$

with

$$
g(z)=\left\{\int_{-\infty}^{z} d w \frac{1}{w^{2}+1} \exp \left[\frac{\mathcal{F}(z)-\mathcal{F}(w)}{a}\right]\right\}^{2}, \quad v^{*}=\alpha-\epsilon
$$

The first integral gives

$$
\int_{-\infty}^{v^{*}} d z \exp \left[\frac{\mathcal{F}(v)-\mathcal{F}(z)}{a}\right] g(z)=\frac{a^{3}}{\left(v^{*}+1\right)^{2}\left[\frac{d \mathcal{F}\left(v^{*}\right)}{d z}\right]^{3}}
$$


In the second integral

$$
g(z)=\frac{a \pi}{\left(\alpha^{2}+1\right)^{2} \frac{d^{2} \mathcal{F}(\alpha)}{d z^{2}}} \exp \left[\frac{2 \mathcal{F}(z)-2 \mathcal{F}(\alpha)}{a}\right] .
$$

from which we get

$$
\int_{v^{*}}^{v} d z \exp \left[\frac{\mathcal{F}(v)+\mathcal{F}(z)-2 \mathcal{F}(\alpha)}{a}\right] \frac{a \pi}{\left(\alpha^{2}+1\right)^{2} \frac{d^{2} \mathcal{F}(\alpha)}{d z^{2}}} .
$$

Integrating in $z$ we obtain

$$
\int_{v^{*}}^{v} d z \exp \left[\frac{\mathcal{F}(z)}{a}\right]=\frac{a}{\frac{d \mathcal{F}(v)}{d z}} \exp \left[\frac{\mathcal{F}(v)}{a}\right]
$$

Integrating in $v$ we obtain

$$
C V^{2}=\frac{8 \chi^{2} \nu^{2} \pi}{\left(\alpha^{2}+1\right)^{2} \frac{d^{2} \mathcal{F}(\alpha)}{d z^{2}}\left(\frac{d \mathcal{F}\left(v_{\max }\right)}{d z}\right)^{2}}\left(\frac{\exp \left[\frac{\mathcal{F}\left(v_{\max }\right)-\mathcal{F}(\alpha)}{a}\right]}{\sqrt{a} K}\right)^{2} .
$$

Using Eq. B6) we obtain

$$
C V=1-\nu \tau_{r p}
$$

which corresponds to the CV of the ISIs of a Poisson process with dead time, with rate $\nu$ and refractory period $\tau_{r p}$. We validated this result numerically in Figs. $8 \mathrm{D}$ and $10 \mathrm{C}$.

\section{Scaling relations preserving firing in the strong coupling limit}

In this section we use the simplified expressions derived above to define scaling relations of $a$ with $K$ which preserves neural response in the strong coupling limit. Importantly, the scaling defined here depends on the operating regime of the neuron, i.e. on the asymptotic value of $\mu$.

In the limit of large $K$, terms in Eq. (A8) can be written as

$$
\tau^{-1}=a K \nu_{X}(1+\eta g \gamma), \quad \omega^{-1}=\nu_{X}(1+\eta g \gamma), \quad \chi^{-1}=\nu_{X}\left(1+\eta g^{2} \gamma\right),
$$

while $\mu, \varepsilon_{\mathcal{D}}, \varepsilon_{\mathcal{S}}, v_{\max }, \alpha$ and the function $\mathcal{F}(x)$ are independent of $K, a$ and $\nu_{E}$. We have shown in the previous section that the single neuron transfer function is given by

$$
\frac{1}{\nu}=\tau_{r p}+\frac{\mathcal{Q}}{\nu_{E}}
$$

with

$$
Q= \begin{cases}\left(\frac{1}{\sqrt{a} K} \exp \frac{\mathcal{F}\left(v_{\max }\right)-\mathcal{F}(\alpha)}{a}\right) \sqrt{\frac{\pi\left(1+\eta g^{2} \gamma\right)}{(1+\eta g \gamma)^{3}\left(\alpha^{2}+1\right)}} \frac{v_{\max }^{2}+1}{\left|v_{\max }-\alpha\right|} & \text { for } \mu<\theta \\ \frac{1}{a K(1+\eta g \gamma)} \log \left(\frac{\mu-\theta}{\mu-V_{r}}\right) & \text { for } \mu>\theta\end{cases}
$$

For $\mu>\theta$, the parameters $a$ and $K$ in Eq. B20 appear only in the combination $a K$. It follows that a rescaling

$$
a \sim \frac{1}{K}
$$

leaves invariant the neural response for large $K$. For $\mu<\theta$, Eq. (B20), and hence the transfer function, is invariant under the rescaling

$$
K \sim \frac{1}{\sqrt{a}} \exp \left[\frac{\mathcal{F}\left(v_{\max }\right)-\mathcal{F}(\alpha)}{a}\right]
$$


A

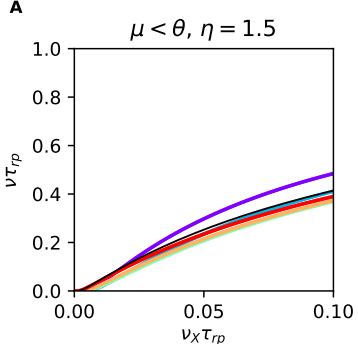

D

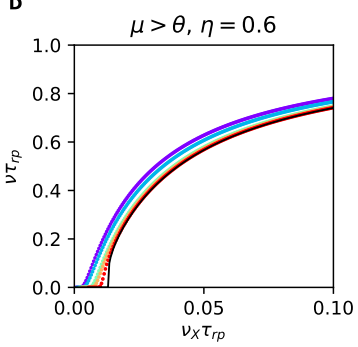

B

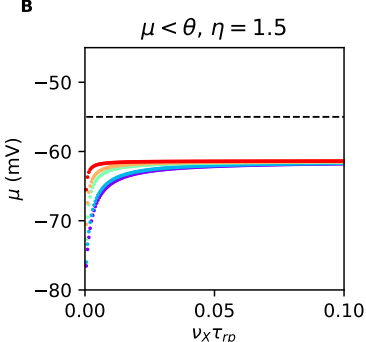

$\mathbf{E}$

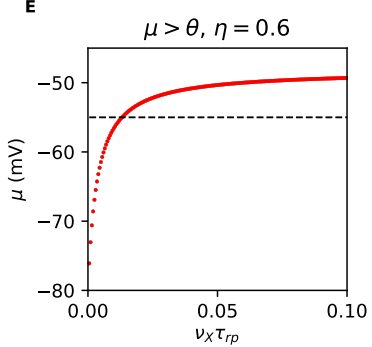

c

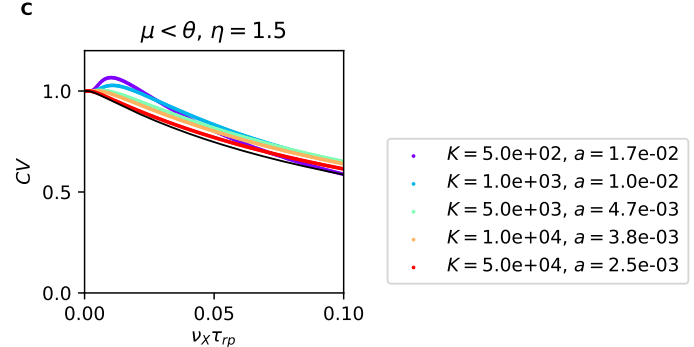

F

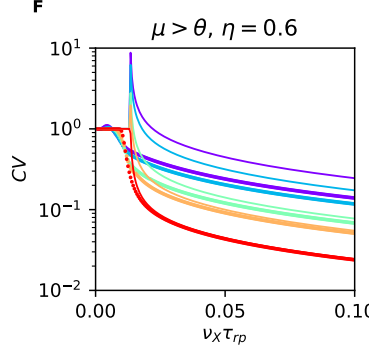

$K=5.0 e+02, a=2.0 e-02$ $K=1.0 \mathrm{e}+03, a=1.0 \mathrm{e}-02$ $K=5.0 \mathrm{e}+03, a=2.0 \mathrm{e}-03$ $K=1.0 \mathrm{e}+04, a=1.0 \mathrm{e}-03$ $K=5.0 \mathrm{e}+04, a=2.0 \mathrm{e}-04$

FIG. 10. Scaling relationships preserving firing in the large $K$ limit. Colored dots represent mean field transfer function $(\mathbf{A}, \mathbf{B}), \mathrm{CV}(\mathbf{C}, \mathbf{D})$ and membrane potential $(\mathbf{E}, \mathbf{F})$ obtained from Eqs. A13, A14 and (A8), respectively. Different colors correspond to different values of $a$ and $K$ which are scaled according to Eqs. (B22) (first row) and (B21) (second row). Mean field predictions are well described by the relevant approximated expressions (continuous lines). For $\mu<\theta$ transfer function and $C V$ are described by Eqs. $\mathrm{B} 22$ (A) and (B17) (C); both quantities are invariant as $K$ increases. For $\mu>\theta$, transfer function and $C V$ are described by Eqs. (B21) $(\mathbf{A})$ and $(\mathrm{B} 9)(\mathbf{C})$; note that, as explained in the text, the firing is preserved while the $C V$ becomes smaller as $K$ increases (different line colors correspond to different values of $K$ ). Parameters: $g=12$; $\gamma=1 / 4$.

In Fig. 10A,D we show neural responses computed for different values of $K$ with $a$ rescaled according to Eqs (B21) or (B22); as predicted the network transfer function remains invariant as $K$ increases. Note that the response remains nonlinear in the limit of large $K$; we will show in the next section that in the network case, because of the self consistency relation, nonlinearities are suppressed by the scaling relation.

Finally, from Fig. $10 \mathrm{C}, \mathrm{F}$, we see that the rescaling preserves the $C V$ for $\mu<\theta$ and suppresses it for $\mu>\theta$. In the case $\mu<\theta$, the $C V$ is given by Eq. (B17). This expression shows that the scaling relation of Eq. (B22) also leaves invariant the $C V$. Interestingly, in some parameter regime, the $C V$ in Figs. $8 \mathrm{D}$ and $10 \mathrm{C}$ shows a non-monotonic behavior with $\nu_{X}$ which is not captured by Eq. (B17). In particular, a $C V$ above one 1 is observed when $\mu$ is below the reset $V_{r}$. As pointed out in [76, this supra-Poissonian firing is explained by the fact that, when $\mu<V_{r}$, spiking probability is higher just after firing that it is afterwards. In agreement with this interpretation, we find that the non-monotonic behavior of the $C V$ is disappears in the large $K$ limit, where the region of inputs for which $\mu<V_{r}$ becomes negligible. Thus, our analysis shows that the irregularity of firing is preserved in the strong coupling limit of a single neuron with $\mu<\theta$.

In the case $\mu>\theta$, the $C V$ is given by Eq. (B9). This expression shows that the scaling relation of Eq. (B21) produces a $C V$ which decreases as $1 / K$ in the strong coupling limit. It follows that, in a single neuron with $\mu>\theta$, the strong coupling limit produces finite firing that is regular.

Starting from the next section we will focus our attention to network of conductance-based neurons. Since we are interested in describing the irregular firing observed in the cortex, we will focus our study on networks with $\mu<\theta$. 


\section{Appendix C: Firing rate and scaling relation in leaky integrate-and-fire neuron models with} voltage-dependent currents

In the main text, we have shown that, when coupling is strong and $a \ll 1$, the response of a single LIF neuron with conductance-based synapses is well approximated by Eq. (12), i.e. Kramers escape rate. Using this expression, we have show that the scaling relation of Eq. (14) allows finite firing in single neuron and in networks of neurons. Here, we show that the first order approximation of this scaling, i.e. $a \sim 1 / \log (K)$, appears also in neuron models with additional biophysical details, such as spike generating currents [43] and voltage-gated subthreshold currents [23], as long as coupling is strong, $a$ is small, and synapses are conductance-based.

We consider integrate-and-fire models featuring voltage-dependent currents, indicated here as $\phi(V)$, and conductance-based synapses. In these models, the membrane potential dynamics can be written as

$$
\mathcal{C}_{j} \frac{d V_{j}}{d t}=-\sum_{A=L, E, I} g_{A}^{j}\left(V_{j}-E_{A}\right)+\psi(V) .
$$

In the leaky integrate-and-fire model (LIF), $\psi(V)=0$ and Eq. (C1) reduces to Eq. (1) analyzed in the main text. In the exponential integrate-and-fire model (EIF) [43, the function $\psi(V)=\Delta T g_{L} \exp [(V-\theta) / \Delta T]$ describes the spike generation current; in this model, once the membrane potential crosses the threshold $\theta$ it diverges to infinity in finite time. The current generated by inward rectifier voltage-gated channels, such as the one recently reported in [23, is captured by an expression of the form $\psi(V)=-g_{i n}(V)\left(V-E_{i n}\right)$, where $g_{i n}(V)$ and $E_{i n}$ represent the conductance and the reversal potential of the channels, respectively; in the case of [23], $1 / g_{i n}(V)$ was shown to well approximated by a linear increasing function of $V$.

The dynamics Eq. (C1), following an approach analogous to the one we used for the derivation of Eq. (4), can be approximated by

$$
\tau \frac{d V}{d t}=-\frac{\partial \mathcal{H}(V)}{\partial V}+\sigma \sqrt{\tau} \zeta, \quad \mathcal{H}(V)=\frac{1}{2}(V-\mu)^{2}-\frac{\tau}{\tau_{L} g_{L}} \int^{V} \psi(x) d x
$$

where $\zeta$ is a white noise term, with zero mean and unit variance density, while $\tau, \mu$ and $\sigma(V)$ are as in Eq. (5). In what follows, as in the main text, we use the effective time constant approximation 39, i.e. we neglect the multiplicative component of the noise term in Eq. (C2), and make the substitution $\sigma(V) \rightarrow \sigma\left(\mu^{*}\right)$, where $\mu^{*}$ is the mean value of the membrane potential dynamics.

The firing rate of a neuron following Eq. C2 can be computed exactly using Eq. A5 and is given by

$$
\nu=\left[\tau_{r p}+\frac{2 \tau}{\sigma^{2}} \int_{-\infty}^{\infty} d x \int_{\max \left(V_{r}, x\right)}^{\infty} \exp \left(\frac{\mathcal{H}(z)-\mathcal{H}(x)}{\sigma^{2}}\right) d z\right]^{-1} .
$$

In what follows, we provide a more intuitive derivation of the single neuron response, which is valid in the biologically relevant case of $a \ll 1$. The function $\mathcal{H}$ in Eq. $\mathrm{C2}$ ) can be though of as an energy function which drives the dynamics of the membrane potential. In the case of LIF neurons, $\mathcal{H}$ is a quadratic function with a minimum at $V=\mu$. In neuron models with a spike generation current, such as the EIF model [43, the shape of the function $\mathcal{H}$ far from threshold is qualitatively similar to that of the LIF model (with a minimum at $V=\mu^{*}$ ), but becomes markedly different close to threshold, where the potential energy has a maximum at $V=\theta^{*}$ and goes to $-\infty$ for $V>\theta^{*}$. Here, we focus on the case in which additional subthreshold voltage-gated currents do not lead to additional minima of the energy function, a scenario that can happen with potassium inward-rectifier currents (e.g. see 777 chapter 4.4.3). In models in which $\mathcal{H}$ has a single minimum in the subthreshold range at $\mu^{*}$, and a maximum at $\theta^{*}$, the firing rate of a neuron when input noise is small (i.e. when $a \ll 1$ ) can again be computed using Kramers escape rate, which gives the average time it take for the membrane potential to go from $\mu^{*}$ to $\theta^{*}$, (see [42] section 5.5.3)

$$
\frac{1}{\nu}-\tau_{r p}=\frac{2 \pi \bar{\tau} \bar{\Upsilon}}{a K \nu_{X}} \exp \left(\frac{\bar{\Delta}}{a}\right)
$$

where

$$
\bar{\Upsilon}=\left(\left.\left.\frac{d^{2} \mathcal{H}}{d V^{2}}\right|_{\theta^{*}} \frac{d^{2} \mathcal{H}}{d V^{2}}\right|_{\mu^{*}}\right)^{-\frac{1}{2}}, \quad \bar{\Delta}=\frac{\mathcal{H}\left(\theta^{*}\right)-\mathcal{H}\left(\mu^{*}\right)}{\bar{\sigma}}, \quad \bar{\tau}=a K \nu_{X} \tau, \quad \bar{\sigma}=\frac{\sigma}{\sqrt{a}},
$$

while - indicates quantities that remain of order 1 in the small $a$ limit, provided the external inputs $\nu_{X}$ are at least of order $1 /\left(a K \tau_{L}\right)$. Eq. (C4) is the generalization of Eq. 12 to the case of integrate-and-fire neuron models with voltage-dependent currents; it shows that, at the dominant order, finite firing emerges if $a \sim 1 / \log (K)$. Moreover, Eq. (C4) shows that corrections to the logarithmic scaling depend on the specific type of voltage-dependent currents used in the model. 


\section{Appendix D: Calculations in the strong coupling regime - Networks}

In this section, we show how the results on the strong coupling limit of single neuron response can be generalized to the network case. First, we analyze the problem in the case in which excitatory and inhibitory neurons have the same biophysical properties (model A). In this model we start by discussing the results using the effective time constant approximation, and then discuss the full results. Then we study the case in which excitatory and inhibitory neurons have different biophysical properties (model B).

\section{Model A, effective time constant approximation}

As discussed in the main text, the network response in model A with the effective time constant approximation is obtained solving the self-consistency condition given by $\sqrt{19}$ and Eq. 10 . At strong coupling, this condition can be simplified to the form of Eq. (12). In the strong coupling limit, when $\nu_{X} \gg 1 / a K \tau_{L}$ and $\nu \gg 1 / \tau_{r p}$, the right hand side of Eq. 10 depends on $\nu$ and $\nu_{X}$ only through their ratio. Therefore, we look for solutions of the simplified self-consistency condition with a Taylor expansion

$$
\frac{\nu}{\nu_{X}}=\sum_{k=1}^{k=\infty} \rho_{k} x^{k-1}, \quad x=\tau_{r p} \nu_{X}
$$

Keeping only terms up to first order in $x$, the self-consistency condition becomes

$$
\frac{1}{\rho_{1}}-\left(1+\frac{\rho_{2}}{\rho_{1}^{2}}\right) x=\mathcal{Q}\left(\rho_{1}\right)+\left.\rho_{2} \frac{d \mathcal{Q}(y)}{d y}\right|_{y=\rho_{1}} x
$$

from which we find

$$
\rho_{1}=\frac{1}{\mathcal{Q}\left(\rho_{1}\right)}
$$

The solution of Eq. (D2) provides the linear component of the network response; this is preserved in the strong coupling limit with an expression analogous to Eq. (14) but with

$$
\frac{r_{E}}{\nu_{X}}=1+\rho_{1}, \quad \frac{r_{I}}{\nu_{X}}=\rho_{1} .
$$

This uniquely defines a scaling between $a$ and $K$ (see Fig. $3 \mathrm{~A}$ for an example of the scaling function). We test the validity of our result in Fig. $3 \mathrm{~B}$. The numerical analysis shows that, as $K$ increases, the scaling relation prevents saturation and suppression of the network response. However, unlike what happens in the single neuron case, the shape of the transfer function is not preserved and becomes increasingly linear as $K$ becomes larger. This is analogous to what happens in the balanced state model 17, 8, 10, 72, where the network transfer function becomes linear in the strong coupling limit. For the case under investigation here, we can understand this suppression of nonlinearities by looking at the second order terms in the expansion of Eq. (D1). Keeping the dominant contribution in $a$, we find

$$
\rho_{2} \sim a \frac{\rho_{1} \bar{\sigma}^{2}}{2 \bar{v}_{\max }\left(\bar{\sigma} \frac{d \mu}{d y}+(\theta-\mu) \frac{d \bar{\sigma}}{d y}\right)} .
$$

Hence $\rho_{2}$ goes to zero as $a$ decreases, producing a linear transfer function. This follows directly from the self-consistency relation and is not present in the single neuron case, where in fact a nonlinear transfer function is observed in the large $K$ limit. Fig. 3B shows that linearity is reached really slowly with $K$; this follows directly from Eq. (D3) where the suppression of nonlinear terms is controlled by $a$, which slowly goes to zero with $K$ (approximately logarithmically).

\section{Model A, multiplicative noise}

In this section, we generalize the approach used above, relaxing the effective time constant approximation. As discussed in Appendix B, Eq. A13 in the strong coupling limit becomes

$$
\frac{1}{\nu}=\tau_{r p}+\frac{\mathcal{Q}}{\nu_{X}}
$$


A

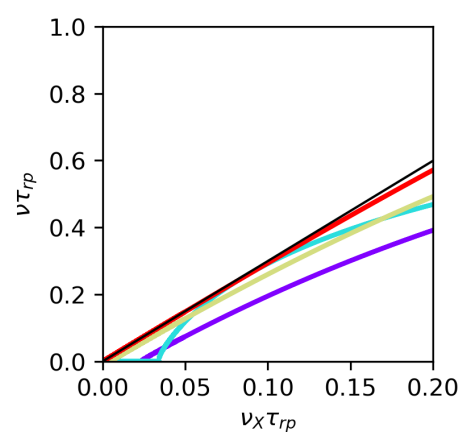

B

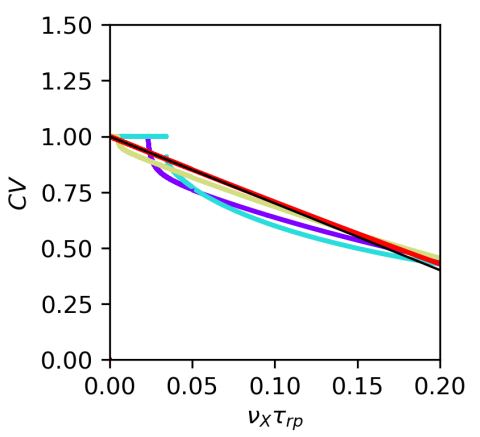

C

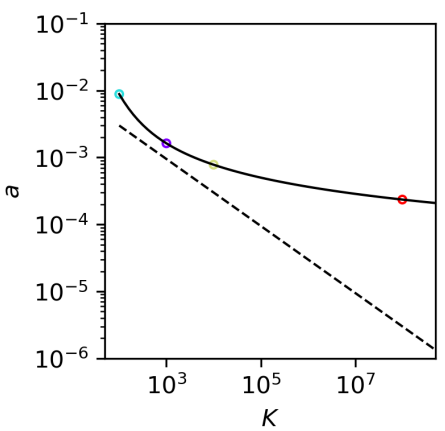

D

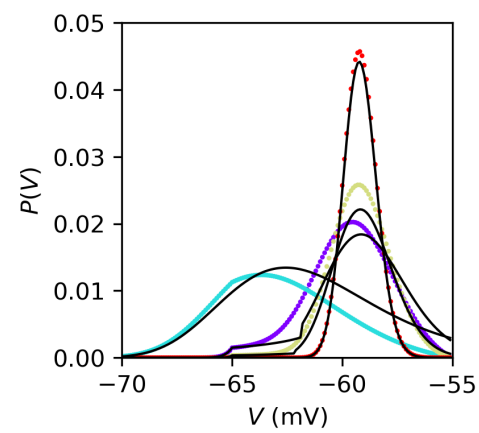

$K=1.0 \mathrm{e}+04, a=7.8 \mathrm{e}-04$ $K=1.0 \mathrm{e}+08, a=2.4 \mathrm{e}-04$
E

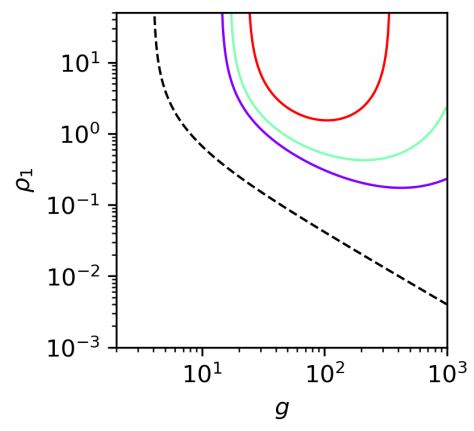

F

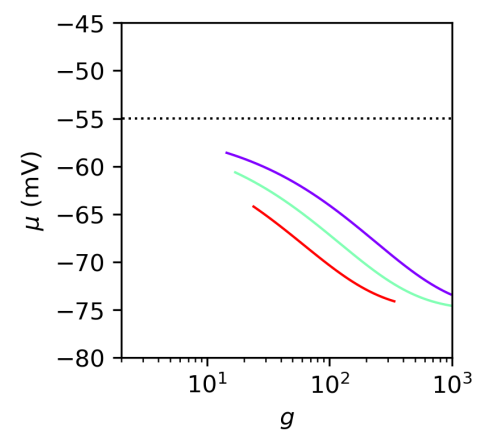

$K=1.0 \mathrm{e}+03, a=1.6 \mathrm{e}-03$ $K=1.0 \mathrm{e}+02, a=8.8 \mathrm{e}-03$

FIG. 11. Strong coupling limit of networks of conductance-based neurons in model A. Numerically computed network transfer function $(\mathbf{A}), C V(\mathbf{B})$ and probability distribution of the membrane potential (D) obtained from Eqs. (D4), A12 and (B17). Different colors correspond to different values of $a$ and $K$ which have been changed according to the scaling relation (D10) (C). As $K$ increases the network transfer function and $C V$ converges to the expression derived in the main text (black lines). Note that, unlike the case of single neuron, the network transfer functions becomes linear. The probability distribution of the membrane potential becomes Gaussian and slowly converges to a delta function. Panels (E) and (F) show the network gain and membrane potential for different values of $a$ at fixed $K$. Note that, unlike what happens in current-based networks (black dashed lines), the gain is not monotonic with $g$. Simulation parameters are as in Fig. 8. in panels A-D $g=20$.

with

$$
\mathcal{Q}=\left\{\frac{1}{\sqrt{a} K} \exp \left[\frac{\mathcal{F}\left(v_{\max }\right)-\mathcal{F}(\alpha)}{a}\right]\right\} \sqrt{\frac{\pi\left[1+\frac{\nu}{\nu_{X}}\left(1+g^{2} \gamma\right)\right]}{\left(1+\frac{\nu}{\nu_{X}}(1+g \gamma)\right)^{3}\left(\alpha^{2}+1\right)}} \frac{v_{\max }^{2}+1}{\left|v_{\max }-\alpha\right|},
$$

and

$$
\begin{array}{r}
\tau^{-1}=a K \omega^{-1}, \quad \omega^{-1}=\nu_{X}\left[1+\frac{\nu}{\nu_{X}}(1+g \gamma)\right], \quad \chi^{-1}=\nu_{X}\left[1+\frac{\nu}{\nu_{X}}\left(1+g^{2} \gamma\right)\right], \\
\mu=\frac{E_{E}+\frac{\nu}{\nu_{X}}\left(E_{E}+g \gamma E_{I}\right)}{1+\frac{\nu}{\nu_{X}}(1+g \gamma)}, \quad \mathcal{E}_{\mathcal{S}}=\frac{E_{E}+\frac{\nu}{\nu_{X}}\left(E_{E}+g^{2} \gamma E_{I}\right)}{1+\frac{\nu}{\nu_{X}}\left(1+g^{2} \gamma\right)}, \\
\mathcal{E}_{\mathcal{D}}=\frac{\left(E_{E}-E_{I}\right) \sqrt{\left(1+\frac{\nu}{\nu_{X}}\right) \frac{\nu}{\nu_{X}} g^{2} \gamma}}{1+\frac{\nu}{\nu_{X}}\left(1+g^{2} \gamma\right)} .
\end{array}
$$

Here we assumed $a K \gg 1 / \tau_{L} \nu_{X}$ so that the function $\mathcal{Q}$ depends on $\nu$ and $\nu_{X}$ only through the combination $\nu / \nu_{X}$. We will show below that a scaling relation analogous to that of single neurons holds, hence for $K$ large enough 
$a K \gg 1 / \tau_{L} \nu_{X}$ is automatically implemented. To solve the self consistency condition, we express the firing rate $\nu$ with a Taylor expansion

$$
\tau_{r p} \nu=\sum_{k=1}^{k=\infty} \rho_{k} x^{k}, \quad x=\tau_{r p} \nu_{X}
$$

Note that in Eq. (D7) we assumed $\rho_{0}=0$, we will come back to this point at the end of the section. Under this assumption $y:=\nu / \nu_{X}=\sum_{k=1}^{k=\infty} \rho_{k} x^{k-1}$ and the function $\mathcal{Q}$ depends only on powers of the dimensionless variable $x$. Keeping only terms up to first order in $x$, Eq. (D4) becomes

$$
\frac{1}{\rho_{1}}-\left(1+\frac{\rho_{2}}{\rho_{1}^{2}}\right) x=2\left(\rho_{1}\right)+\left.\rho_{2} \frac{d \mathcal{Q}(y)}{d y}\right|_{y=\rho_{1}} x
$$

from which we find

$$
\rho_{1}=\frac{1}{Q\left(\rho_{1}\right)}
$$

The solution of Eq. (D9) provides the linear component of the network response, i.e. its gain; we will discuss this function in more detail at the end of this section.

From Eq. (D9) we find that the network gain $\rho_{1}$ is preserved in the strong coupling limit if the factor

$$
\frac{1}{\sqrt{a} K} \exp \left[\frac{\mathcal{F}\left(v_{\max }\right)-\mathcal{F}(\alpha)}{a}\right]
$$

is constant. Eq. D10) uniquely defines a scaling between $a$ and $K$ (see Fig. 11C for an example of the scaling function). We test the validity of the scaling in Fig. 11] as follows: given a set of parameters $a, K$ and $\rho_{1}$, we compute numerically the transfer function from Eq. A13), then we increased $K$, determined the corresponding change in $a$ using Eq. (D10) and compute again the transfer function; results of this procedure are shown in Fig. 11A. The numerical analysis shows that, as $K$ increases our scaling relation prevent saturation and the network response remains finite.

As in the case with diffusion approximation, the shape of the transfer function is not preserved by the scaling and an increasing linear response is observed. We can understand this suppression of nonlinearities by looking at the second order terms in the expansion of Eq. (D4); we find

$$
\rho_{2}=\frac{-\rho_{1}^{2}}{\rho_{1} \frac{d \log (Q(y))}{d y}+1},
$$

and, keeping the dominant contribution in $1 / a$ at the denominator,

$$
\rho_{2} \sim \frac{-a \rho_{1}}{\left.\frac{d \mathcal{F}\left(v_{\max }(y), y\right)}{d y}\right|_{\rho_{1}}+\left.\frac{d \mathcal{F}(\alpha(y), y))}{d y}\right|_{\rho_{1}}} .
$$

Hence $\rho_{2}$ goes to zero as $a$ decreases, producing a linear transfer function. The nonlinearities at low rate in Fig. 11A (e.g. see red and yellow lines) show that our assumption $\rho_{0}=0$ is not valid in general. However it turns out that the above defined scaling relation suppresses also these nonlinearities in the limit of strong coupling (e.g. blue and cyan lines).

We now characterize the dependency of the transfer function gain, i.e. its slope, on network parameters. For fixed network parameters, the network gain $\rho_{1}$ is defined as the solution of Eq. (D9); solutions as a function of $a$ and $g$ are shown in Fig. 11E. At fixed values of $a$, the gain initially decreases as $g$ increases and, for $g$ large enough, the opposite trend appears. This behavior is due to two different effects which are produced by the increase of $g$ : on one hand, it increases the strength of recurrent inhibition; on the other hand, it decreases the equilibrium membrane potential $\mu$ and bring it closer to the inhibitory reversal potential $E_{i}$, which in turn weakens inhibition (see Fig. 11F). Fig. $11 \mathrm{E}$ shows that the gain is finite only for a finite range of the parameter $g$; divergences appear because recurrent inhibition is not sufficiently strong to balance excitation. At small $g$, the unbalance is produced by week efficacy of inhibitory synapses; at large $g$, inhibition is suppressed by the approach of the membrane potential to the reversal point of inhibitory synapses. Increasing the value of $a$ produces an upward shift in the curve and, at the same time, decreases the range of values in which the gain is finite. The observed decrease in gain generated at low values of $g$ is 
observed also networks of current-based neurons [10] where the gain is found to be $1 /(g \gamma-1)$. Finally, we note that the difference between conductance and current-based model decreases with $a$.

To conclude this analysis, we give an approximated expression of the probability distribution of the membrane potential of Eq. A12 which, in the strong coupling limit, becomes

$$
P(V)=\frac{\nu \omega}{\left|v_{\max }-\mu\right|}\left[\frac{u\left(V_{\max }\right)^{2}+1}{u(V)^{2}+1}\right] \frac{e^{\frac{\mathscr{I}\left(v_{\max }\right)-\mathscr{F}(V)}{a}}}{a K}
$$

where $V_{\max }$ is the value of the membrane potential $V$ which maximizes the integrand of Eq. (A12) while the function $u()$ has been defined in Eq. A15). Examples of the probability distribution and the corresponding approximated expressions are given in Fig. 11D.

\section{Model B, multiplicative noise}

In this section we generalize the results obtained so far to the case of networks with excitatory and inhibitory neurons with different biophysical properties.

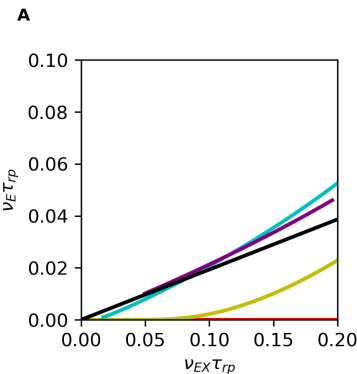

D

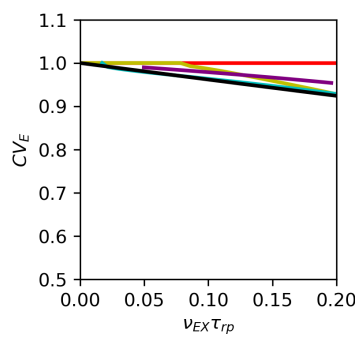

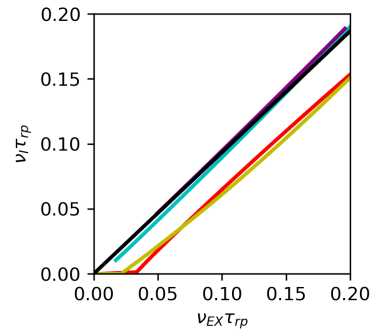

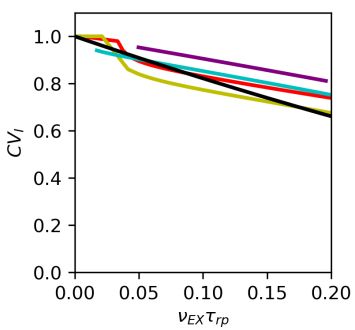

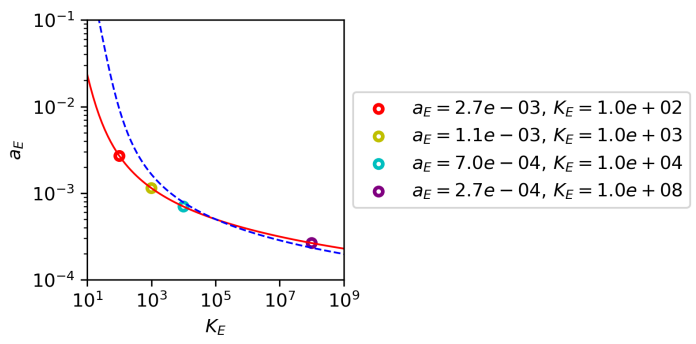

$\mathbf{F}$

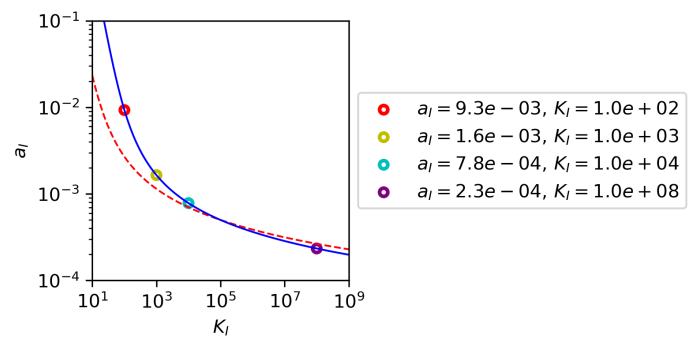

FIG. 12. Limit of large $K$ for networks, model B. Firing rate and $C V$ of excitatory and inhibitory neurons in a network predicted by the mean field model for different values of inputs and $K$; the expected asymptotic behavior is shown in black. On the left $(\mathbf{C}, \mathbf{F})$, we show the corresponding scaling relations with dots associated to the connectivity parameters. Simulations parameter: the two populations have $g_{e}=20.0$ and $g_{i}=19.0$; for both populations the $a=0.0005$ for $K=10^{5}$; other parameters as in Fig. 8

a. Model definition

Here we take into account the diversity of the two types of neurons with

$$
\tau_{j}=\tau_{E}, \quad a_{j m}=a_{E X}, a_{E E}, a_{E I} ;
$$

for excitatory neurons and

$$
\tau_{j}=\tau_{I}, \quad a_{j m}=a_{I X}, a_{I E}, a_{E E} ;
$$

for inhibitory neurons. We use the parametrization

$$
\begin{array}{r}
a_{E X}=a_{E}, \quad a_{E E}=a_{E}, \quad a_{E I}=g_{E} a_{E}, \\
a_{I X}=a_{I}, \quad a_{I E}=a_{I}, \quad a_{I I}=g_{I} a_{I},
\end{array}
$$


and

$$
\begin{array}{r}
K_{E X}=K_{E}, \quad K_{E E}=K_{E}, \quad K_{E I}=\gamma_{E} K_{E} \\
K_{I X}=K_{I}, \quad K_{I E}=K_{I}, \quad K_{I I}=\gamma_{I} K_{I}
\end{array}
$$

Eq. (1) becomes

$$
\left\{\begin{array}{l}
\tau_{E} \frac{d V_{E}}{d t}=-\left(V_{E}-\mu_{E}\right)-\sigma_{E}\left(V_{E}\right) \sqrt{\tau_{E}} \zeta_{E} \\
\tau_{I} \frac{d V_{I}}{d t}=-\left(V_{I}-\mu_{I}\right)-\sigma_{I}\left(V_{I}\right) \sqrt{\tau_{I}} \zeta_{I}
\end{array}\right.
$$

The expressions for excitatory neurons are

$$
\begin{array}{r}
\tau_{E}^{-1}=\tau_{L, E}^{-1}+a_{E} K_{E} \omega_{E}^{-1}, \quad \omega_{E}^{-1}=\nu_{E X}+\nu_{E}+g_{E} \gamma_{E} \nu_{I}, \\
\mu_{E}=\tau_{E}\left\{E_{L}+a_{E} K_{E} \tau_{L, E}\left[\nu_{E X} E_{E}+\nu_{E} E_{E}+\nu_{I} g_{E} \gamma_{E} E_{I}\right]\right\}, \\
\sigma_{E}^{2}=a_{E}^{2} K_{E} \frac{\tau_{E}}{\chi_{E}}\left[\left(V-\varepsilon_{\mathcal{S}, E}\right)^{2}+\mathcal{E}_{\mathcal{D}, E}^{2}\right], \quad \chi_{E}^{-1}=\nu_{E X}+\nu_{E}+g_{E}^{2} \gamma_{E} \nu_{I} \\
\left.\mathcal{E}_{S, E}=\chi_{E}\left[\nu_{E X} E_{E}+\nu_{E} E_{E}+\nu_{I} g_{E}^{2} \gamma_{E} E_{I}\right)\right], \\
\mathcal{E}_{\mathcal{D}, E}=\chi_{E} \sqrt{\left(\nu_{E X}+\nu_{E}\right) g_{E}^{2} \gamma_{E} \nu_{I}}\left(E_{E}-E_{I}\right) ;
\end{array}
$$

analogous expressions are valid for inhibitory neurons.

The firing rate is given by solving a system of two equations

$$
\left\{\begin{array}{l}
\frac{1}{\nu_{E}}-\tau_{r p}=\frac{2 \chi_{E}}{a_{E}^{2} K_{E}} \int_{v_{\min , E}}^{v_{\max , E}} d v \int_{-\infty}^{v} d x \frac{1}{x^{2}+1} \exp \left[\frac{\mathcal{F}_{E}(v)-\mathcal{F}_{E}(x)}{a_{E}}\right] \\
\frac{1}{\nu_{I}}-\tau_{r p}=\frac{2 \chi_{I}}{a_{I}^{2} K_{I}} \int_{v_{\min , I}}^{v_{\max , I}} d v \int_{-\infty}^{v} d x \frac{1}{x^{2}+1} \exp \left[\frac{\mathcal{F}_{I}(v)-\mathcal{F}_{I}(x)}{a_{I}}\right]
\end{array}\right.
$$

with

$$
\begin{array}{r}
\mathcal{F}_{E}(x)=\frac{2 \chi_{E}}{a_{E} K_{E} \tau_{E}}\left[\frac{1}{2} \log \left(x^{2}+1\right)-\alpha_{E} \arctan (x)\right], \\
v_{m i n, E}=\frac{V_{r}-\mathcal{E}_{\mathcal{S}, E}}{\mathcal{E}_{\mathcal{D}, E}}, \quad v_{\max , E}=\frac{\theta-\mathcal{E}_{\mathcal{S}, E}}{\mathcal{E}_{\mathcal{D}, E}}, \quad \alpha_{E}=\frac{\mu_{E}-\mathcal{E}_{\mathcal{S}, E}}{\mathcal{E}_{\mathcal{D}, E}} .
\end{array}
$$

and analogous expressions for the inhibitory population. The probability distribution of the membrane potential and the $C V$ are straightforward generalizations of Eqs. A12 and A14.

\section{b. Scaling analysis}

We parametrize inputs to the two populations as $\nu_{E X}$ and $\nu_{I X}=\eta \nu_{E X}$. Using an analysis analogous to the one depicted above, we obtain a simplified expression for the self-consistency Eq. (D20) that is

$$
\left\{\begin{array}{l}
\frac{1}{\nu_{E}}-\tau_{r p}=\frac{Q_{E}\left(\nu_{E} / \nu_{E X}, \nu_{I} / \nu_{E X}\right)}{\nu_{E X}} \\
\frac{1}{\nu_{I}}-\tau_{r p}=\frac{Q_{i}\left(\nu_{E} / \nu_{E X}, \nu_{I} / \nu_{E X}\right)}{\nu_{E X}}
\end{array}\right.
$$

where

$$
\mathcal{Q}_{E}=\left[\frac{1}{\sqrt{a_{E}} K_{E}} \exp \frac{\mathcal{F}_{E}\left(v_{\max , E}\right)-\mathcal{F}_{E}\left(\alpha_{E}\right)}{a_{E}}\right] \sqrt{\frac{\pi\left[1+\frac{\nu_{E}}{\nu_{E X}}+g_{E}^{2} \gamma_{E} \frac{\nu_{I}}{\nu_{E X}}\right]}{\left[1+\frac{\nu_{E}}{\nu_{E X}}+g_{E} \gamma_{E} \frac{\nu_{I}}{\nu_{E X}}\right]^{3}\left(\alpha_{E}^{2}+1\right)}} \frac{v_{\max , E}^{2}+1}{\left|v_{\max , E}-\alpha_{E}\right|},
$$

and

$$
Q_{I}=\left[\frac{1}{\sqrt{a_{I}} K_{I}} \exp \frac{\mathcal{F}_{I}\left(v_{\max , I}\right)-\mathcal{F}_{I}\left(\alpha_{I}\right)}{a_{I}}\right] \sqrt{\frac{\pi\left[\eta+\frac{\nu_{E}}{\nu_{E X}}+g_{I}^{2} \gamma_{I} \frac{\nu_{I}}{\nu_{E X}}\right]}{\left[\eta+\frac{\nu_{E}}{\nu_{E X}}+g_{I} \gamma_{I} \nu_{I} \nu_{E X}\right]^{3}\left(\alpha_{I}^{2}+1\right)}} \frac{v_{\max , I}^{2}+1}{\left|v_{\max , I}-\alpha_{I}\right|} .
$$


We investigate the solution in the strong coupling limit using an expansion

$$
\tau_{r p} \nu_{E}=\sum_{k=1}^{k=\infty} \rho_{k}^{E} x^{k}, \quad \tau_{r p} \nu_{I}=\sum_{k=1}^{k=\infty} \rho_{k}^{I} x^{k}, \quad x=\tau_{r p} \nu_{E X},
$$

and obtain

$$
\left\{\begin{array}{l}
\rho_{1}^{E}=\frac{1}{\mathcal{Q}_{E}\left(\rho_{1}^{E}, \rho_{1}^{I}\right)} \\
\rho_{1}^{I}=\frac{1}{\mathcal{Q}_{I}\left(\rho_{1}^{E}, \rho_{1}^{I}\right)}
\end{array} .\right.
$$

Eq. D26 defines the gain of the excitatory and inhibitory populations. As for model A, requiring that network gain is preserved in the large $K$ limit is equivalent to assuming the products

$$
\frac{1}{\sqrt{a_{j}} K_{j}} \exp \frac{\mathcal{F}_{j}\left(v_{\max , j}\right)-\mathcal{F}_{j}\left(\alpha_{j}\right)}{a_{j}}
$$

constant; these constraints defines how synaptic strength should scale with $K$ to preserve the response gain. We note that, since $\mathcal{F}_{j}\left(v_{\max , j}\right)-\mathcal{F}_{j}\left(\alpha_{j}\right)$ is different for the two populations, in the general case there are two different scalings for the two populations; in Fig 12 we verify this prediction.

\section{Appendix E: Simulations vs theory}

All the results showed in the main text are based on the mean field analysis of the network dynamics. in this section we investigate how the predictions of the mean field theory compare to numerical simulations of networks of conductance-based neurons.

Using the simulator Brian2 [66, we simulated the dynamics of networks of spiking neurons defined by Eq. (1). We investigated networks of $N_{E}$ excitatory and $N_{I}$ inhibitory neurons; the two groups were driven by two populations of Poisson units of size $N_{E X}$ and $N_{I X}$, respectively. Simulations were performed for $N_{E}=N_{I}=N_{E X}=N_{I X}=10 K$ and $100 \mathrm{~K}$, with no significant differences between the two. We used uniformly distributed delays of excitatory and inhibitory synapses. Delays were drawn randomly and independently at each existing synapse from uniform distributions in the range $[0,10] \mathrm{ms}$ (E synapses) and $[0,1] \mathrm{ms}$ (I synapses). For fixed network parameters, the dynamics was simulated for 10 seconds with a time step of $10 \mu \mathrm{s}$. We performed simulations for different values of $K$; the values of $a$ was rescaled according to the scaling relation of Eq. (D10). From the resulting activity we measured firing rate, $C V$ and probability distribution of the membrane potential; results are shown in Fig. 13 , Mean field predictions are in qualitative agreement with numerical simulations, and the agreement improves as $a$ decreases. Deviations from mean-field are expected to arise potentially from three factors: (1) Finite size of conductance jumps due to pre-synaptic action potentials; (2) Correlations in synaptic inputs to different neurons in the network due to recurrent connectivity; (3) Temporal correlations in synaptic inputs due to non-Poissonian firing behavior. In our simulations, deviations due to (1) and (2) become small when both $a$ and the connection probability are small. Deviations due to (3) become small when $\nu \ll 1 / \tau_{r p}$, since as shown in Eq. (B17) of Appendix B, the statistics of presynaptic neurons firing tend to those of a Poisson process. As predicted by the mean field analysis, with increasing $K$ (and decreasing $a$ ) the network response becomes linear and approaches the asymptotic scaling; the firing remains irregular, as shown by the $C V$, and the membrane potential becomes Gaussian distributed.

Appendix F: Effects of heterogeneity in the connectivity between neurons

In this section, we describe how fluctuations in single cell properties modify the expressions described above; in particular we investigate the effect of heterogeneities in number of connections per neuron in the simplified framework of model A. The formalism described here is a generalization to networks of conductance-based neurons of the analysis done in refs [56, 78, for networks of current-based neurons.

We assume that the $i$-th neuron in the network receives projections from $K_{X}^{i}, K_{E}^{i}$ and $K_{I}^{i}$ external, excitatory and inhibitory neurons, respectively. These numbers are drown randomly from Gaussian distributions with mean $K(\gamma K)$ and variance $\Delta K^{2}\left(\gamma^{2} \Delta K^{2}\right)$ for excitatory (inhibitory) synapses. Note that $\Delta K^{2}$ is assumed to be sufficiently small so that the probability to generate a negative number can be neglected. Fluctuations in the number of connections 


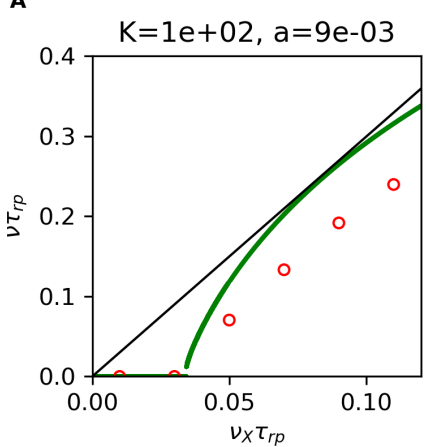

D

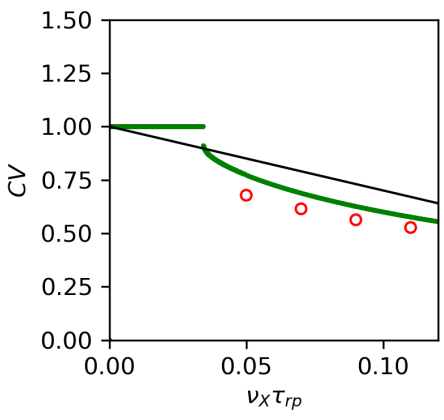

G

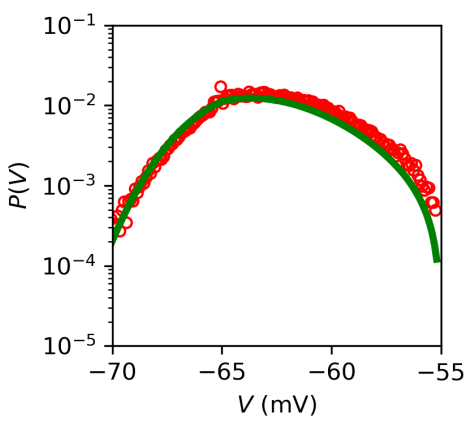

B

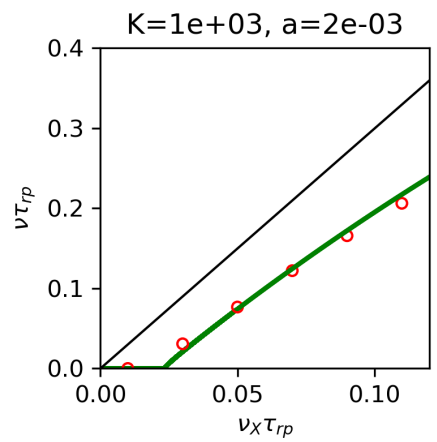

E

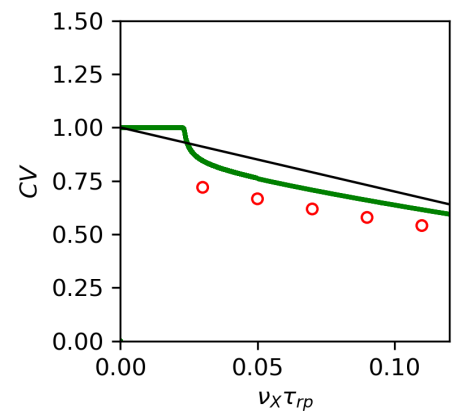

H

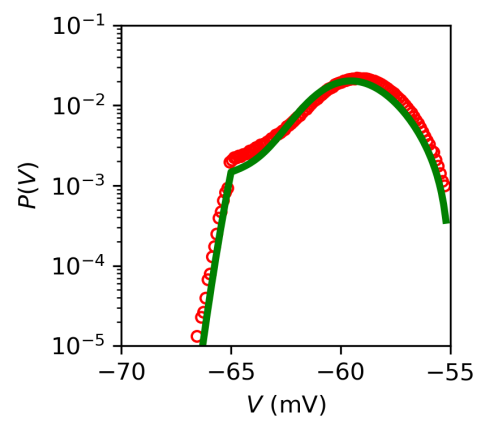

C

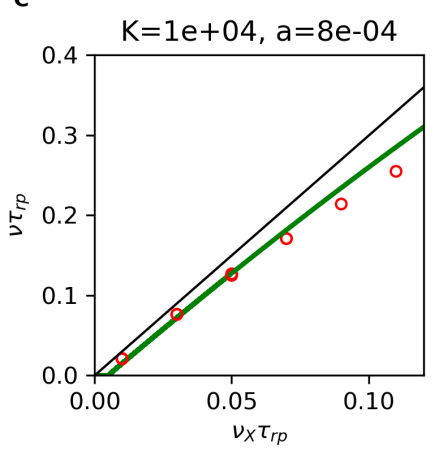

F

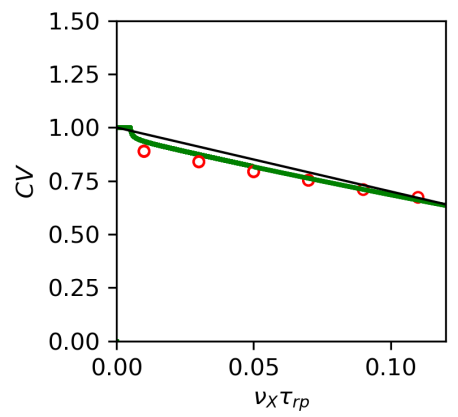

I

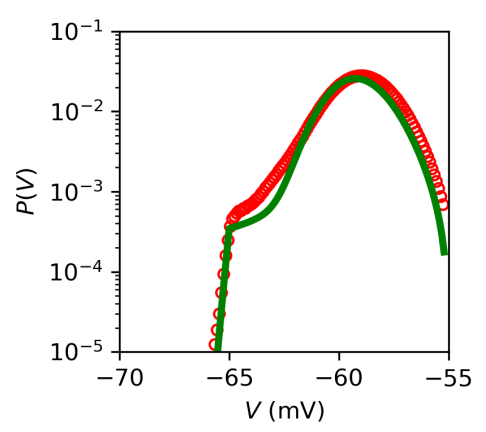

FIG. 13. Comparison of mean field theory and numerical simulations. Network transfer function (first row), $C V$ of ISI distribution (second row) and probability distribution of the membrane potential at $\nu_{E}=0.05 \tau_{r p}$ (third row). In every panel we show mean field prediction (green), results from numerical simulations (red) and value expected in the strong coupling limit (black). Different columns correspond to different values of $K$ and $a$ which were scaled according to Eq. (D10). The agreement between network simulations (red) and mean field predictions (green) improves as $a$ decreases, as expected since we used the diffusion approximation to derive the results. Simulation parameters are: $g=20, N_{E}=N_{I}=N_{E X}=N_{I X}=100 K$.

are expected to produce a distribution of rates in the population, characterized by mean and variance $\nu$ and $\Delta \nu^{2}$. As a result, the rates of incoming excitatory and inhibitory spikes differ from cell to cell and become

$$
\begin{gathered}
K_{E}^{i} r_{E}^{i}=K\left(r_{E}+\Delta_{E} z_{E}^{i}\right), \quad K_{I}^{i} r_{I}^{i}=\gamma K\left(r_{I}+\Delta_{I} z_{I}^{i}\right), \quad r_{E}=\nu+\nu_{X}, \quad r_{I}=\nu, \\
\Delta_{E}^{2}=C V_{K}^{2}\left(\nu^{2}+\nu_{X}^{2}\right)+\frac{\Delta \nu^{2}}{K} \approx C V_{K}^{2}\left(\nu^{2}+\nu_{X}^{2}\right), \quad \Delta_{I}^{2}=C V_{K}^{2} \nu^{2}+\frac{\Delta \nu^{2}}{\gamma K} \approx C V_{K}^{2} \nu^{2} ;
\end{gathered}
$$

where $r_{E, I}$ are the average presynaptic rates and $z_{E, I}^{i}$ are realizations of a quenched normal noise with zero mean and unit variance, fixed in a given realization of the network connectivity. Starting from Eq. (F1), the rate $\nu^{i}$ of the cell is derived as in the case without heterogeneities, the main difference is that it is now a function of the particular realizations of $z_{E}^{i}$ and $z_{I}^{i}$. The quantities $\nu$ and $\Delta \nu^{2}$ are obtained from population averages through the self consistency 
relations

$$
\left\{\begin{array}{l}
\nu=\left\langle\nu\left(z_{E}, z_{I}\right)\right\rangle \\
\Delta \nu^{2}=\left\langle\nu\left(z_{E}, z_{I}\right)^{2}\right\rangle-\nu^{2}
\end{array}\right.
$$

where $\langle$.$\rangle represents the Gaussian average over the variables z_{E}$ and $z_{I}$. Once $\nu$ and $\Delta \nu^{2}$ are known, the probability distribution of firing rate in the population is given by

$$
P(\nu)=\frac{1}{2 \pi} \int_{-\infty}^{\infty} d z_{E} d z_{I} e^{-z_{E}^{2} / 2} e^{-z_{I}^{2} / 2} \delta\left[\nu-\nu\left(z_{E}, z_{I}\right)\right]
$$

As showed in the main text (Fig. 4A), Eq. (F3) captures quantitatively the heterogeneity in rates observed in numerical simulations.

In the large $K$ (small $a$ ) limit, the mathematical expressions derived above simplify significantly. First, as long as the parameter $\mu^{i}$ of the i-th neuron is below threshold, its rate is given by an expression analogous to Eq. (12) which, for small $\Delta_{E, I}$, can be written

$$
Q_{i}=\mathcal{Q} \exp \left(\Gamma z_{i}\right), \quad \Gamma^{2}=\left(\frac{\partial v_{\max }^{2}}{\partial r_{E}} \Delta_{E}\right)^{2}+\left(\frac{\partial v_{\max }^{2}}{\partial r_{I}} \Delta_{I}\right)^{2}
$$

where $z^{i}$ is generated from a Gaussian random variable with zero mean and unit variance. Moreover, if responses are far from saturation, the single rate can be written as

$$
\nu_{i}=\frac{\nu_{X}}{Q_{i}}=\nu_{0} \exp \left(-\Gamma z_{i}\right), \quad \Gamma^{2}=\Omega^{2} \frac{C V_{K}^{2}}{a^{2}}, \quad \Omega^{2}=\left[\left(a \frac{\partial v_{\max }^{2}}{\partial\left(r_{E} / \nu_{X}\right)}\right)^{2}\left(\rho^{2}+1\right)^{2}+\left(a \frac{\partial v_{\max }^{2}}{\partial\left(r_{I} / \nu_{X}\right)}\right)^{2} \rho^{2}\right]
$$

where $\nu_{0}$ is the rate in the absence of quenched noise (i.e. Eq. (20) of the main text). It is easy to show that, in Eq. (F5), $\Omega^{2}$ is independent of $a, K$ and $\nu_{X}$ in the large $K$ (small $a$ ) limit. Finally, as noted in [56], if the single neuron rate can be expressed as an exponential function of a quenched variable $z$, Eq. (F3) can be integrated exactly and the distribution of rates is lognormal and given by

$$
P(\nu)=\frac{1}{\sqrt{2 \pi} \Gamma \nu} \exp \left(-\frac{\left(\log (\nu)-\log \left(\nu_{0}\right)\right)^{2}}{2 \Gamma^{2}}\right) .
$$

Therefore, when the derivation of Eq. $\overline{F 5}$ is valid, rates in the network should follow a log normal distribution, with parameters given by

$$
\left\{\begin{array}{l}
\nu=\nu_{0} \exp \left(\frac{\Gamma^{2}}{2}\right) \\
\Delta \nu^{2}=\nu^{2}\left[\exp \left(\frac{\Gamma^{2}}{2}\right)-1\right]
\end{array}\right.
$$

For $\Gamma^{2} \ll 1$, we find $\Delta \nu / \nu \approx \Gamma / 2$ which scales linearly with $C V_{K}$, consistent with numerical results shown in Fig. 4 C.

\section{Appendix G: Finite synaptic time constants}

In this section, we discuss the effect of synaptic time constant on single neuron and network responses. First, we derive an approximated expression for the single neuron membrane time constant; we then compute approximated expressions which are valid for different values of the ratio $\tau_{S} / \tau$; at the end of the section, we discussing the response of networks of neurons with large $\tau_{S} / \tau$.

The single neuron membrane potential dynamics is given by

$$
\left\{\begin{array}{l}
\mathcal{C}_{j} \dot{V}_{j}(t)=-g_{L}^{j}\left(V_{j}-E_{L}\right)-\sum_{A=E, I} g_{A}^{j}(t)\left(V_{j}-E_{A}\right), \\
\tau_{E} \dot{g}_{E}^{j}=-g_{E}^{j}+g_{L}^{j} \tau_{E} \sum_{m} a_{j m} \sum_{n} \delta\left(t-t_{m}^{n}-D\right) \\
\tau_{I} \dot{g}_{I}^{j}=-g_{I}^{j}+g_{L}^{j} \tau_{I} \sum_{m} a_{j m} \sum_{n} \delta\left(t-t_{m}^{n}-D\right)
\end{array}\right.
$$


Using the effective time constant approximation 39, we have

$$
\left\{\begin{array}{l}
\mathcal{e} \dot{V}=-g_{0}(V-\mu)-g_{E F}\left(\mu-E_{E}\right)-g_{I F}\left(\mu-E_{I}\right) \\
\tau_{E} \dot{g}_{E F}=-g_{E F}+\sigma_{E} \sqrt{\tau_{E}} \zeta_{E} \\
\tau_{I} \dot{g}_{I F}=-g_{I F}+\sigma_{I} \sqrt{\tau_{I}} \zeta_{I}
\end{array}\right.
$$

where $g_{A F}$ represents the fluctuating component of the conductance $g_{A}$, i.e.

$$
g_{A}(t)=g_{A 0}+g_{A F}(t)
$$

and

$$
\begin{array}{r}
\left\langle\zeta_{A}(t) \zeta_{B}\left(t^{\prime}\right)\right\rangle=\delta_{A, B} \delta\left(t-t^{\prime}\right), \quad g_{0}=g_{L}+g_{E 0}+g_{I 0}, \\
g_{A 0}=a_{A} \tau_{A} R_{A}, \quad \sigma_{A}^{2}=a_{A}^{2} \tau_{A} R_{A}
\end{array}
$$

We are interested in stationary response, we introduce the term

$$
z=\left(\mu-E_{E}\right) g_{E F}+\left(\mu-E_{I}\right) g_{I F}
$$

with derivative

$$
\dot{z}=\left(\mu-E_{E}\right) \frac{-g_{E F}+\sigma_{E} \zeta_{E}}{\tau_{E}}+\left(\mu-E_{I}\right) \frac{-g_{I F}+\sigma_{I} \zeta_{I}}{\tau_{I}}
$$

Since we are interested in understanding the effect of an additional time scale, we can simplify the analysis assuming a unique synaptic time scale $\tau_{E}=\tau_{I}=\tau_{S}$ and obtain

$$
\begin{array}{r}
\tau_{S} \dot{z}=-z+\sigma_{z} \sqrt{\tau_{S}} \zeta \\
\sigma_{z}^{2}=\sigma_{E}^{2}\left(\mu-E_{E}\right)^{2}+\sigma_{I}^{2}\left(\mu-E_{I}\right)^{2}
\end{array}
$$

To have the correct limit for $\tau_{S} \rightarrow 0$, we impose $a_{A}=a_{A 0} \tau_{L} / \tau_{S}$, where $a_{A 0}$ is the value of the synaptic efficacy in the limit of instantaneous synaptic time scale. With these assumptions the system equation becomes

$$
\left\{\begin{array}{l}
\tau \frac{d V}{d t}=-(V-\mu)-\sigma \sqrt{\frac{\tau}{\tau_{S}}} z \\
\tau_{s} \frac{d z}{d t}=-z+\sqrt{\tau_{S}} \zeta
\end{array}\right.
$$

One can check that in the limit $\tau_{S} \rightarrow 0$, the equations become analogous to those of the main text with $\eta=z / \sqrt{\tau_{S}}$. In what follows, we provide approximated expressions for the single neuron transfer function in three regimes: small time constant [67], large time constant [69], and for intermediate values [70]. We also note that a numerical procedure to compute the firing rate exactly for any value synaptic time constant was introduced recently, using Fredholm theory [79].

\section{Single neuron transfer function for different values of $\tau_{S} / \tau$}

For $\tau_{S} / \tau \ll 1$, as shown in [67, the firing rate can be computed with a perturbative expansion and is given by

$$
\frac{1}{\nu}=\tau \sqrt{\pi} \int_{\tilde{v}_{\text {min }}}^{\tilde{v}_{\max }} d x(1+\operatorname{erf}(x)), \quad \tilde{v}(x)=\frac{x-\mu}{\sigma}-\tilde{\alpha} \sqrt{\frac{\tau_{S}}{\tau}} .
$$

with $\bar{\alpha}=-\zeta(1 / 2) \approx 1.46$. As shown in Fig. 14 Eq. G9 generates small corrections around the prediction obtained with instantaneous synapses, and captures well the response for values $\tau_{S} / \tau \lesssim 0.1$.

For $\tau_{S} / \tau \approx 1$, as shown in [70] using Rice formula [80, the single neuron firing rate is well approximated by the rate of upward threshold crossing of the membrane potential dynamics without reset. Starting from Eq. (G8) and using the results of [70], we obtain

$$
\nu=\frac{1}{2 \pi \sqrt{\tau \tau_{S}}} \exp \left[-v_{\max }^{2}\left(1+\frac{\tau_{S}}{\tau}\right)\right]
$$



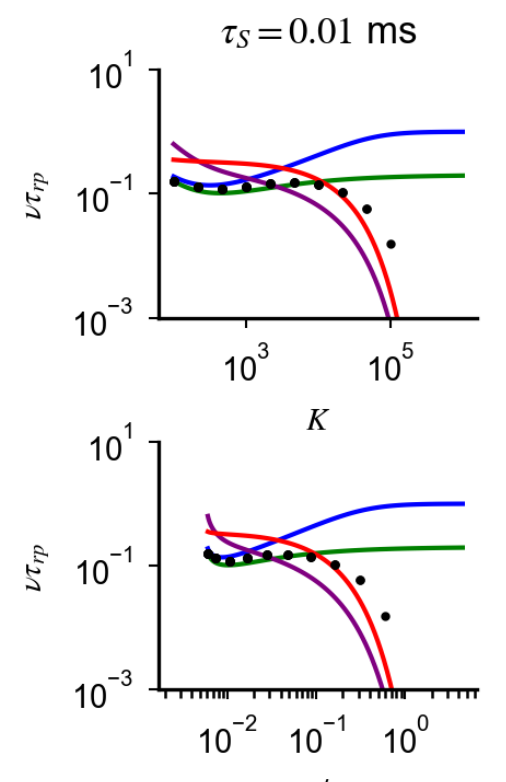

\begin{tabular}{|c|c|c|c|c|c|}
\hline$\tau_{S} / \tau$ & & $\tau_{S} / \tau$ & & & $\tau_{S} / \tau$ \\
\hline$\longrightarrow \tau_{S} / \tau=0$ & $\longrightarrow \tau_{S} / \tau \ll 1$ & $\longrightarrow \tau_{S} / \tau \sim 1$ & - & $\tau_{S} / \tau \gg 1$ & - simulation \\
\hline
\end{tabular}

For $\tau_{S} / \tau \gg 1$, as shown in [69, the neuron fires only when fluctuations of $z$ are large enough for $V$ to be above

FIG. 14. Synaptic time constant suppresses single neuron response in the strong coupling limit. Single neuron response for different values of $K$, with $a$ rescaled according to Eq. 144. Rates are plotted as a function of $K$ (first row) and $\tau_{S} / \tau$ (second row); different columns correspond to different synaptic time constant $\tau_{S}$ (title). As $K$ increases, because of the synaptic time constant $\tau_{S}$ non-negligible compared to the membrane time constant $\tau$, rates computed numerically from Eq. 23) (black dots) depart from the prediction of Eq. 10 (green). The dependency of the rate on $K$ is captured by Eq. (G9) (blue) for small values of $\tau_{S} / \tau$ and by Eq. (G11) (red) for large values of $\tau_{S} / \tau$. This decay cannot be prevented by a new scaling relation of $a$ with $K$ and provides an upper bound to how much coupling can be increased while preserving response. Simulations parameter: $a=0.006$ for $K=10^{3}, g=12, \eta=1.46$. threshold; the corresponding rate is given by

$$
\nu=\int_{v_{\max } / \epsilon}^{\infty} d w \frac{e^{-w^{2}}}{\sqrt{\pi}} \frac{1}{\tau_{r p}+\tau \log \left(\frac{v_{\min }-\epsilon w}{v_{\max }-\epsilon w}\right)}, \quad \epsilon=\sqrt{\frac{\tau}{\tau_{S}}}
$$

As shown in Fig. 14 Eq. G11] captures the response for values $\tau_{S} / \tau \gtrsim 1$ and predicts a strong suppression of response at larger $\tau_{S} / \tau$.

Higher order terms in the $\tau_{S} / \tau$ expansion could be computed using the approach described in [79]. However, Fig. 14 shows that Eqs. G9 G11) are sufficient to capture quantitatively responses observed in numerical simulations for different regimes of $\tau_{S} / \tau$. Eqs. (G9 G11) show that the single neuron response is a nonlinear function of input rates, this nonlinearity prevents a scaling relation between $a$ and $K$ to rescue the suppression observed in Fig. 14 and Fig. 64.

\section{Network response for $\tau_{S} / \tau$ larger than one}

In this section, we study responses in networks of neurons with large $\tau_{S} / \tau$. As in the case of instantaneous synapses, the network response can be obtained solving the self-consistency relation given by the single neuron transfer function using input rates

$$
r_{E}=\nu_{X}+\nu, \quad r_{I}=\nu
$$



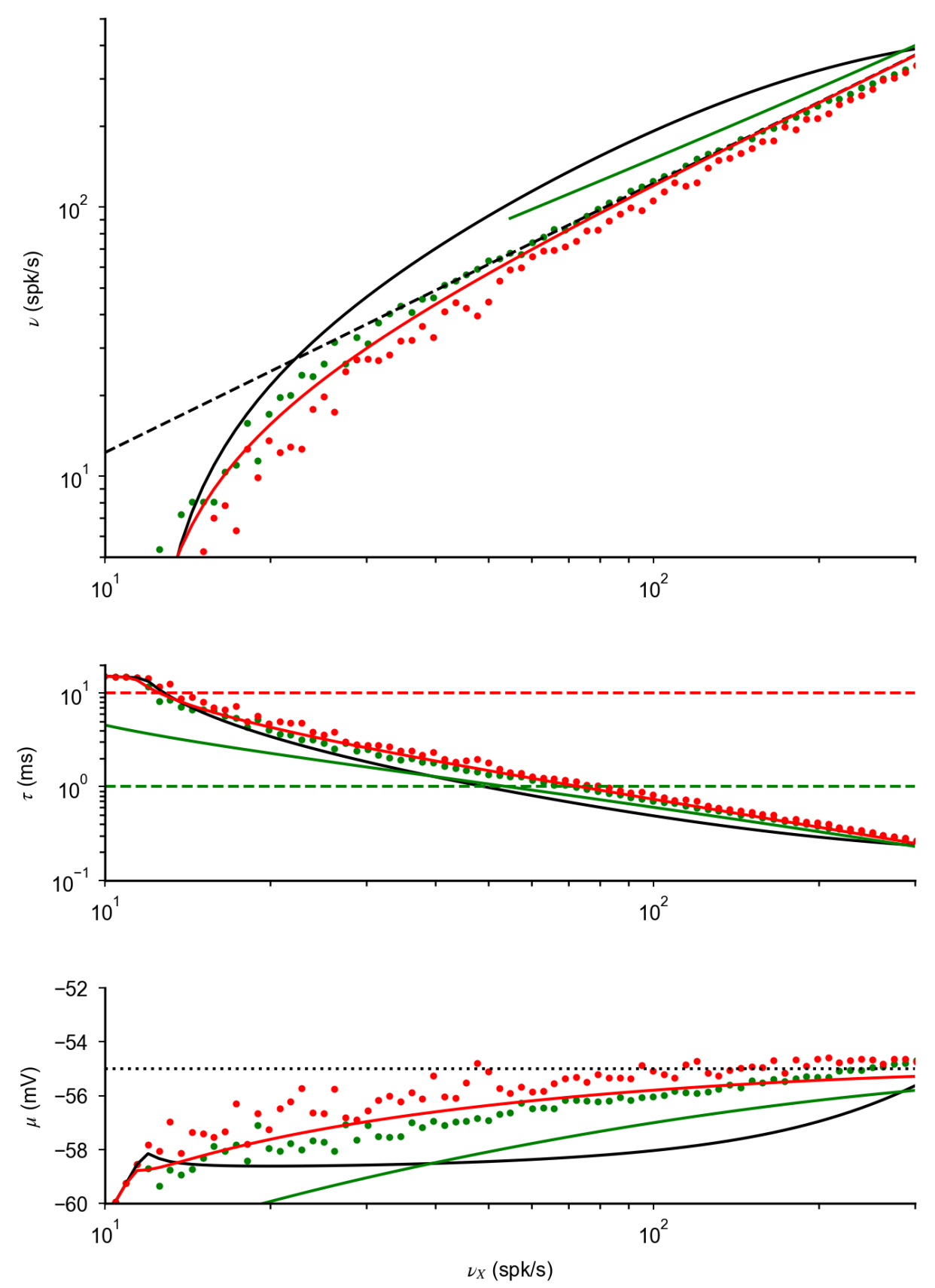

In particular, solutions of the implicit equation generated by Eq. G11 give the network response in the region of

FIG. 15. Approximation of network response for large $\tau_{S} / \tau$. Plots analogous to Fig. 6B,C of the main text. Dots represent network response as a function of input rate $\nu_{X}$, computed numerically from Eqs. (1), (23) for $\tau_{S}=1 \mathrm{~ms}$ (green) and $\tau_{S}=10 \mathrm{~ms}$ (red). Continuous lines correspond to the prediction obtained with instantaneous synapses (black) and for large synaptic time constant (Eqs. G125, G13), colored lines). As explained in the text, the latter predictions are valid only for large $\tau_{S} / \tau$; because of this, we plotted only values obtained for $\tau_{S} / \tau>1$. For $\tau_{S} / \tau \gg 1$, the network response is well describe by Eq. 21 of the main text. inputs for which $\tau_{S} / \tau \gg 1$. In this region of inputs, assuming coupling to be strong, the implicit equation becomes

$$
\nu=\frac{\sqrt{\tau / \tau_{S}}}{\tau_{r p} v_{\max } \sqrt{\pi}} \exp \left(-v_{\max }^{2} \frac{\tau_{S}}{\tau}\right) .
$$


Eq. G12, which is validated numerically in Fig. 15 implies that firing is preserved if $v_{\max } \sqrt{\tau_{S} / \tau}$ is of order one, i.e. if

$$
\mu \sim \theta-\sigma \sqrt{\frac{\tau}{\tau_{S}}} \sim \theta-\frac{1}{\sqrt{K}} \frac{\sigma / \sqrt{a}}{\sqrt{\tau_{S}\left[\nu_{X}+\nu(1+g \gamma)\right]}} .
$$

Combining the above equation with the definition of $\mu$, we obtained Eq. 21 , which captures the behavior of network response observed in numerical simulations for $\tau_{S} / \tau \gg 1$ (Fig. 6B and Fig. 15).

Eq. G12 can be used to understand the effect of connection-heterogeneity in networks with large $\tau_{S} / \tau$. In particular, generalizing the analysis of Appendix $\mathrm{F}$ we found that rates in the network, in the limit of small $C V_{K}$ and large $K$, are given by

$$
\nu_{i}=\nu_{0} \exp \left[\Omega_{S} \frac{C V_{K}}{\sqrt{K}} z_{i}\right]
$$

where $\nu_{0}$ is the population average in the absence of heterogeneity (i.e. the solution of Eq. (G12)), and $z_{i}$ is a Gaussian random variable of zero mean and unit variance. The prefactor $\Omega_{S}$, which is independent of $a$ and $K$, and is given by

$$
\Omega_{S}^{2}=\left[\left(\frac{\partial f\left(r_{E}, r_{I}\right)}{\partial r_{E}}\right)^{2}\left(\nu^{2}+\nu_{X}^{2}\right)+\left(\frac{\partial f\left(r_{E}, r_{I}\right)}{\partial r_{I}}\right)^{2} \nu^{2}\right], \quad f\left(r_{E}, r_{I}\right)=\frac{v_{\max }^{2} \tau_{S}}{K \tau} .
$$

Eq. G15 is a generalization of Eq. 22 to the case of large $\tau_{S} / \tau$. It shows that, in this limit, the state of the network is preserved with connection fluctuations up to $C V_{K} \sim 1 / \sqrt{K}$. 